\title{
Knots with unknotting number 1 and essential Conway spheres
}

\author{
C MCA GORDON \\ JOHN LUECKE
}

For a knot $K$ in $S^{3}$, let $\mathbf{T}(K)$ be the characteristic toric sub-orbifold of the orbifold $\left(S^{3}, K\right)$ as defined by Bonahon-Siebenmann. If $K$ has unknotting number one, we show that an unknotting arc for $K$ can always be found which is disjoint from $\mathbf{T}(K)$, unless either $K$ is an EM-knot (of Eudave-Muñoz) or $\left(S^{3}, K\right)$ contains an EM-tangle after cutting along $\mathbf{T}(K)$. As a consequence, we describe exactly which large algebraic knots (ie, algebraic in the sense of Conway and containing an essential Conway sphere) have unknotting number one and give a practical procedure for deciding this (as well as determining an unknotting crossing). Among the knots up to 11 crossings in Conway's table which are obviously large algebraic by virtue of their description in the Conway notation, we determine which have unknotting number one. Combined with the work of Ozsváth-Szabó, this determines the knots with 10 or fewer crossings that have unknotting number one. We show that an alternating, large algebraic knot with unknotting number one can always be unknotted in an alternating diagram.

As part of the above work, we determine the hyperbolic knots in a solid torus which admit a non-integral, toroidal Dehn surgery. Finally, we show that having unknotting number one is invariant under mutation.

57N10; 57M25

\section{Introduction}

Montesinos showed [24] that if a knot $K$ has unknotting number 1 then its double branched cover $M$ can be obtained by a half-integral Dehn surgery on some knot $K^{*}$ in $S^{3}$. Consequently, theorems about Dehn surgery can sometimes be used to give necessary conditions for a knot $K$ to have unknotting number 1 . For instance, $H_{1}(M)$ must be cyclic, and the $\mathbb{Q} / \mathbb{Z}$-valued linking form on $H_{1}(M)$ must have a particular form (Lickorish [19]). If $K$ is a 2-bridge knot, then $M$ is a lens space, and hence, by the Cyclic Surgery Theorem (Culler, Gordon, Luecke and Shalen [5]), $K^{*}$ must be a torus knot. In this way the 2 -bridge knots with unknotting number 1 
have been completely determined (Kanenobu and Murakami [15]). Another example is Scharlemann's theorem that unknotting number 1 knots are prime [28]; this can be deduced from the fact (proved later, however by Gordon and Luecke [9]) that only integral Dehn surgeries can give reducible manifolds (see Zhang [38]). Finally, we mention the recent work of Ozsváth and Szabó [27], in which the Heegaard Floer homology of $M$ is used to give strong restrictions on when $K$ can have unknotting number 1, especially if $K$ is alternating.

The present paper explores another example of this connection. Here, the Dehn surgery theorem is the result of Gordon and Luecke [11] that the hyperbolic knots with nonintegral toroidal Dehn surgeries are precisely the Eudave-Muñoz knots $k(\ell, m, n, p)$ [6]; this gives information about when a knot $K$ whose double branched cover $M$ is toroidal can have unknotting number 1 .

First we clarify the extension of the main result of [11] to knots in solid tori that is described in the Appendix of [11]. In Section 3, we define a family of hyperbolic knots $J_{\varepsilon}(\ell, m)$ in a solid torus, $\varepsilon \in\{1,2\}$ and $\ell, m$ integers, each of which admits a half-integer surgery yielding a toroidal manifold. (The knots $J_{\varepsilon}(\ell, m)$ in the solid torus are the analogs of the knots $k(\ell, m, n, p)$ in the 3-sphere.) We then use [11] to show that these are the only such:

Theorem 4.2 Let $J$ be a knot in a solid torus whose exterior is irreducible and atoroidal. Let $\mu$ be the meridian of $J$ and suppose that $J(\gamma)$ contains an essential torus for some $\gamma$ with $\Delta(\gamma, \mu) \geq 2$. Then $\Delta(\gamma, \mu)=2$ and $J=J_{\varepsilon}(\ell, m)$ for some $\varepsilon, \ell, m$.

This theorem along with the main result of [11] then allows us to describe in Theorem 5.2 the relationship between the torus decomposition of the exterior of a knot $K$ in $S^{3}$ and the torus decompositon of any non-integral surgery on $K$. In particular, Theorem 5.2 says that the canonical tori of the exterior of $K$ and of the Dehn surgery will be the same unless $K$ is a cable knot (in which case an essential torus of the knot exterior can become compressible), or $K$ is a $k(\ell, m, n, p)$, or $K$ is a satellite with pattern $J_{\varepsilon}(\ell, m)$ (in the latter cases a new essential torus is created).

We apply these theorems about non-integral Dehn surgeries to address questions about unknotting number. The knots $k(\ell, m, n, p)\left(J_{\varepsilon}(\ell, m)\right)$ are strongly invertible. Their quotients under the involutions give rise to EM-knots, $K(\ell, m, n, p)$ (EM-tangles, $\mathcal{A}_{\varepsilon}(\ell, m)$ resp.) which have essential Conway spheres and yet can be unknotted (trivialized, resp.) by a single crossing change. Theorem 6.2 descibes when a knot with an essential Conway sphere or 2-torus can have unknotting number 1 . This is naturally stated in the context of the characteristic decomposition of a knot along 
toric 2-suborbifolds given by Bonahon and Siebenmann in [3]. The characteristic torus decomposition of the double branched cover of a knot $K$ corresponds to the characteristic decomposition of the orbifold $O(K)$, where $O(K)$ refers to $S^{3}$ thought of as an orbifold with singular set $K$ and cone angle $\pi$ (see [3]). This decomposition of $O(K)$ is along Conway spheres and along tori disjoint from $K$, the collection of which is denoted $\mathbf{T}(K)$. When $O(K)$ is cut along $\mathbf{T}(K)$, $\operatorname{Seif}(K)$ denotes the components corresponding to Seifert-fibered components of the canonical torus decomposition in the double branched cover. An unknotting arc, $(a, \partial a)$, for $K$ is an arc such that $a \cap K=\partial a$ that guides a crossing move that unknots $K$. Under the correspondence between crossing changes and Dehn surgeries in the double branched cover, Theorem 5.2 becomes

Theorem 6.2 Let $K$ be a knot with unknotting number 1. Then one of the following three possibilities holds.

(1) (a) Any unknotting arc $(a, \partial a)$ for $K$ can be isotoped in $\left(S^{3}, K\right)$ so that $a \cap \mathbf{T}(K)=\varnothing$.

(b) If $\mathbf{T}(K) \neq \varnothing$ and $K$ has an unknotting $\operatorname{arc}(a, \partial a)$ in $\operatorname{Seif}(K)$ then $(a, \partial a)$ is isotopic to an $(r, s)$-cable of an exceptional fiber of $\operatorname{Seif}(K)$, for some $s \geq 1$.

(2) (a) $K$ is an EM-knot $K(\ell, m, n, p)$.

(b) $O(K)$ has a unique connected incompressible 2-sided toric 2-suborbifold $S$, a Conway sphere, $K$ has an unknotting arc $(a, \partial a)$ with $|a \cap S|=1$ (the standard unknotting arc for $K(\ell, m, n, p))$, and $K$ has no unknotting arc disjoint from $S$.

(3) $K$ is the union of essential tangles $\mathcal{P} \cup \mathcal{P}_{0}$, where $\mathcal{P}_{0}$ is an EM-tangle $\mathcal{A}_{\varepsilon}(\ell, m)$ and $\partial \mathcal{P}_{0}$ is in $\mathbf{T}(K)$. Any unknotting arc for $K$ can be isotoped into $\mathcal{P}_{0}$. The standard unknotting arc for $\mathcal{A}_{\varepsilon}(\ell, m)$ is an unknotting arc for $K$.

Scharlemann and Thompson proved [30; 31] that if a satellite knot has unknotting number one, then an unknotting arc can be isotoped off any companion 2-torus. (This follows from Corollary 3.2 of [30] when the genus of $K, g(K)$, is $\geq 2$. When $g(K)=1$, it follows from the proof of Corollary 3.2 of [31], or from Corollary 1 of Kobayashi [17], which say that a knot $K$ has $u(K)=g(K)=1$ if and only if it is a Whitehead double.) The following corollary of Theorem 6.2 can be thought of as a generalization of this result.

Corollary 6.3 Let $K$ be a knot with unknotting number 1, that is neither an EMknot nor a knot with an EM-tangle summand with essential boundary. Let $F$ be an incompressible 2-sided toric 2-suborbifold of $O(K)$. Then any unknotting arc $(a, \partial a)$ for $K$ can be isotoped in $\left(S^{3}, K\right)$ so that $a \cap F=\varnothing$. 
When a knot or link contains an essential Conway sphere, one can perform mutations along that sphere. Boileau asked [16, Problem 1.69(c)] if the unknotting number of a link is a mutation invariant. We prove that it is at least true for knots with unknotting number one.

Theorem 7.1 Having unknotting number 1 is invariant under mutation.

We would like to thank Alan Reid for suggesting that we consider mutation.

We apply our results to the knots that are algebraic in the sense of Conway [4] (see also Thistlethwaite [33]), and which have an essential Conway sphere. We call such a knot $K$ a large algebraic knot. Note that the double branched cover of $K$ is a graph manifold (ie, the union of Seifert fiber spaces identified along their boundaries). Theorem 6.2 gives particularly strong constraints on unknotting arcs for knots in this class.

Theorem 8.2 Let $K$ be a large algebraic knot with unknotting number 1. Then either

(1) any unknotting arc for $K$ can be isotoped into either

(a) one of the rational tangles $\mathcal{R}(p / q)$ in an elementary tangle of type I; or

(b) the rational tangle $\mathcal{R}(p / q)$ in an elementary tangle of type II.

In case (a), the crossing move transforms $\mathcal{R}(p / q)$ to $\mathcal{R}(k / 1)$ for some $k$, and $p / q=\frac{2 s^{2}}{2 r s \pm 1}+k$, where $s \geq 1$ and $(r, s)=1$.

In case (b), the crossing move transforms $\mathcal{R}(p / q)$ to $\mathcal{R}(1 / 0)$, and $p / q=\frac{2 r s \pm 1}{2 s^{2}}$, where $s \geq 1$ and $(r, s)=1$.

(2) (a) $K$ is an EM-knot $K(\ell, m, n, p)$.

(b) $O(K)$ has a unique connected incompressible 2-sided toric 2-suborbifold $S$, a Conway sphere, $K$ has an unknotting arc $a$ with $|a \cap S|=1$ (the standard unknotting arc for $K(\ell, m, n, p))$, and $K$ has no unknotting arc disjoint from $S$.

(3) $K$ is the union of essential tangles $\mathcal{P} \cup \mathcal{P}_{0}$, where $\mathcal{P}_{0}$ is an EM-tangle $\mathcal{A}_{\varepsilon}(\ell, m)$ and $\partial \mathcal{P}_{0}$ is in $T(K)$. Any unknotting arc for $K$ can be isotoped into $\mathcal{P}_{0}$. The standard unknotting arc for $\mathcal{A}_{\varepsilon}(\ell, m)$ is an unknotting arc for $K$.

In Section 10 we apply Theorem 8.2 to the knots in Conway's tables [4] of knots up to 11 crossings that can be immediately seen to be large algebraic by virtue of their description in terms of Conway's notation. There are 174 such knots, and we show that exactly 24 of them have unknotting number 1 . In particular, combining our results with 
those of Ozsváth and Szabó, the knots with 10 or fewer crossings that have unknotting number 1 are now completely determined (see [27]).

It follows from Theorem 8.2 that the unknotting number 1 question is decidable for large algebraic knots.

Theorem 11.2 There is an algorithm to decide whether or not a given large algebraic knot $K$, described as a union of elementary marked tangles (Figure 8.1) and 4-braids in $S^{2} \times[0,1]$, has unknotting number 1 , and, if so, to identify an unknotting crossing move.

We remark that the algorithm in Theorem 11.2 is straightforward to carry out in practice.

Finally, in Section 12, we consider large algebraic knots which are alternating and show

Theorem 12.5 Let $K$ be an alternating large algebraic knot with unknotting number 1 . Then $K$ can be unknotted by a crossing change in any alternating diagram of $K$.

The authors would like to thank Mario Eudave-Munõz for pointing out a gap in the original proof of Lemma 2.2 in the case that the double branched cover of $K$ is a Seifert fiber space. Also, the first named author wishes to acknowledge partial support for this work by the National Science Foundation (grant DMS-0305846).

\section{Preliminaries}

For us, a tangle will be a pair $(B, A)$ where $B$ is $S^{3}$ with the interiors of a finite number $(\geq 1)$ of disjoint 3 -balls removed, and $A$ is a disjoint union of properly embedded arcs in $B$ such that $A$ meets each component of $\partial B$ in four points. Two tangles $\left(B_{1}, A_{1}\right)$ and $\left(B_{2}, A_{2}\right)$ are homeomorphic if there is a homeomorphism of pairs $h:\left(B_{1}, A_{1}\right) \rightarrow\left(B_{2}, A_{2}\right)$.

A marking of a tangle $(B, A)$ is an identification of each pair $(S, S \cap A)$, where $S$ is a component of $\partial B$, with $\left(S^{2}, Q=\{N E, N W, S W, S E\}\right)$. A marked tangle is a tangle together with a marking. Two marked tangles are equivalent if they are homeomorphic by an orientation-preserving homeomorphism that preserves the markings.

A tangle $\left(B^{3}, A\right)$ in the 3 -ball is essential if $S^{2}-A$ is incompressible in $B^{3}-A$.

Let $\mathcal{T}=(B, A)$ be a knot in $S^{3}$ or a tangle. A Conway sphere in $\mathcal{T}$ is a 2 -sphere $S \subset$ int $B$ such that $S$ meets $A$ transversely in four points. $S$ is essential if $S-A$ is incompressible in $B-A$ and $(S, S \cap A)$ is not pairwise parallel in $(B, A)$ to $\left(S_{0}, S_{0} \cap A\right)$ for any component $S_{0}$ of $\partial B$.

Algebraic 83 Geometric Topology, Volume 6 (2006) 
A rational tangle is a marked tangle that is homeomorphic to the trivial tangle in the 3-ball, $\left(D^{2}, 2\right.$ points $) \times I$. As marked tangles, rational tangles are parametrized by $\mathbb{Q} \cup\{1 / 0\}$. We denote the rational tangle corresponding to $p / q \in \mathbb{Q} \cup\{1 / 0\}$ by $\mathcal{R}(p / q)$. We will adopt the convention of Eudave-Muñoz [7] for continued fractions Thus $\left[a_{1}, a_{2}, \ldots, a_{n}\right]$ will denote the rational number $\frac{p}{q}=a_{n}+\frac{1}{a_{n-1}+\frac{1}{\cdots+\frac{1}{a_{1}}}}$. We will sometimes write $\mathcal{R}(p / q)$ as $\mathcal{R}\left(a_{1}, \ldots, a_{n}\right)$.

Let $\mathcal{T}=\left(B^{3}, A\right)$ be a tangle in the 3-ball. A slope of $\mathcal{T}$ is the isotopy class (rel $\partial$ ) of an embedded arc $\tau$ in $\partial B^{3}$ such that $\partial \tau \subset A \cap \partial B^{3}$. Given a marking on $\mathcal{T}$, the slopes of $\mathcal{T}$ are in 1-1 correspondence with $\mathbb{Q} \cup\{1 / 0\}$ (via the double branched cover, $S^{1} \times S^{1}$, of $\partial B^{3}$ along $A \cap \partial B^{3}$ ). If $\mathcal{T}$ is rational, then $A$ defines a slope on $\partial B^{3}$. The rational number corresponding to this slope is that assigned to $\mathcal{T}$ in the preceding paragraph, $p / q$. If $\frac{p_{1}}{q_{1}}, \frac{p_{2}}{q_{2}}$ are slopes on some tangle $\mathcal{T}$, then the distance between these slopes, denoted $\Delta\left(\frac{p_{1}}{q_{1}}, \frac{p_{2}}{q_{2}}\right)$, is $\left|p_{1} q_{2}-p_{2} q_{1}\right|$, and is the minimal intersection number between the corresponding isotopy classes in the double branched cover of $\partial B^{3}$ along $A \cap \partial B^{3}$.

Definition An alternating diagram of a marked tangle in $B^{3}$ is said to be positive (negative, resp.) if the first crossings encountered from the boundary (with pictured marking) are as shown in Figure 2.1. An alternating diagram of a marked tangle in $S^{2} \times I$ is said to be positive (negative, resp.) if filling it with $\mathcal{R}(1 / 0)$ gives a positive (negative, resp.) diagram of a tangle in $B^{3}$.
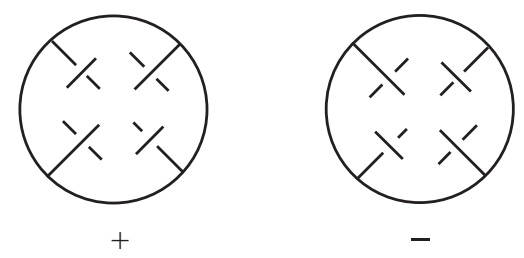

Figure 2.1

By our conventions, then, $\mathcal{R}(p / q)$ has a positive alternating diagram when $p / q>0$.

Let $\mathcal{M}(*, *)$ be the tangle in the thrice-punctured 3-sphere illustrated in Figure 2.2. If $\alpha, \beta \in \mathbb{Q} \cup\{1 / 0\}$, then $\mathcal{M}(\alpha, \beta)$ will denote the tangle in the 3-ball obtained by inserting rational tangles $\mathcal{R}(\alpha), \mathcal{R}(\beta)$ into $A, B$ respectively (with respect to the markings of $\partial A$ and $\partial B$ given by Figure 2.2). Similarly, $\mathcal{M}(\alpha, *)(\operatorname{resp} . \mathcal{M}(*, \beta))$ will denote the tangle in $S^{2} \times I$ obtained by inserting $\mathcal{R}(\alpha)$ (resp. $\mathcal{R}(\beta)$ ) into $A$ (resp. $B)$. 


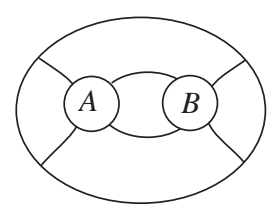

Figure 2.2

If $\alpha, \beta \in \mathbb{Q}-\mathbb{Z}$ then $\mathcal{M}(\alpha, \beta)$ is a Montesinos tangle of length 2 . Note that transferring horizontal twists between $A$ and $B$ shows that $\mathcal{M}(\alpha+m, \beta-m)=\mathcal{M}(\alpha, \beta)$ for all $m \in \mathbb{Z}$.

In general we will denote the double branched cover of a tangle $\mathcal{T}$ by $\widetilde{\mathcal{T}}$. However, we will denote $\widetilde{\mathcal{M}}(*, *)$ by $D^{2}(*, *)$; it is homeomorphic to $P \times S^{1}$, where $P$ is a pair of pants. Similarly, denote the double branched cover of $\mathcal{M}(p / q, *)$ by $D^{2}(p / q, *)$. If $q>1$ this is a Seifert fiber space over the annulus with one exceptional fiber of multiplicity $q$. Finally, the double branched cover of $\mathcal{M}\left(p_{1} / q_{1}, p_{2} / q_{2}\right)$ is $D^{2}\left(p_{1} / q_{1}, p_{2} / q_{2}\right)$; if $q_{1}, q_{2}>1$ this is a Seifert fiber space over the disk with two exceptional fibers of multiplicities $q_{1}$ and $q_{2}$.

Let $\mathcal{S}(*, * ; *, *)$, the square tangle, be the marked tangle shown in Figure 2.3; it is the union of two copies of $\mathcal{M}(*, *)$. If $\alpha, \beta, \gamma, \delta \in \mathbb{Q} \cup\{\infty\}$, then $\mathcal{S}(\alpha, \beta ; \gamma, \delta)$ is the knot or link obtained by inserting the corresponding rational tangle into $A, B, C, D$ respectively.

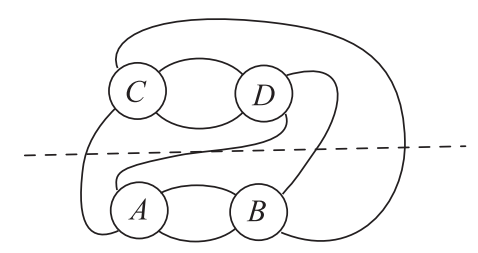

Figure 2.3: $\mathcal{S}(*, * ; *, *)$

Lemma $2.1 \mathcal{S}(\alpha, \beta ; *, *) \cong \mathcal{S}(\beta, \alpha ; *, *)$ by a homeomorphism whose restriction to $\partial C(\partial D)$ is rotation through $180^{\circ}$ about the horizontal axis.

Proof This follows by rotating Figure 2.4 through $180^{\circ}$ about the vertical axis shown, using the fact that a rational tangle is unchanged by rotation through $180^{\circ}$ about the vertical axis.

To state the next lemma, let $D_{8}$ be the order 8 dihedral group of all permutations of $\{\alpha, \beta, \gamma, \delta\}$ that preserve the partition $\{\{\alpha, \beta\},\{\gamma, \delta\}\}$. 


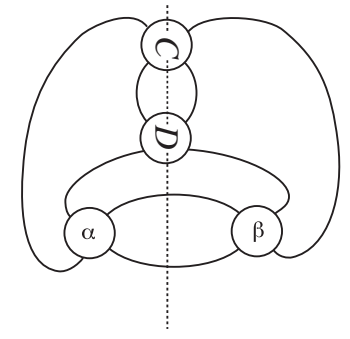

Figure 2.4: $\mathcal{S}(\alpha, \beta ; *, *)$

\section{Lemma 2.2}

(1) $\mathcal{S}(\alpha, \beta ; \gamma, \delta)=\mathcal{S}(\alpha+m, \beta-m ; \gamma+n, \delta-n)$ for all $m, n \in \mathbb{Z}$.

(2) $\mathcal{S}(\alpha, \beta ; \gamma, \delta)=\mathcal{S}(\pi(\alpha), \pi(\beta) ; \pi(\gamma), \pi(\delta))$ for all $\pi \in D_{8}$.

(3) $\mathcal{S}(-\alpha,-\beta ;-\gamma,-\delta)=-\mathcal{S}(\alpha, \beta ; \gamma, \delta)$.

If $\alpha, \beta, \gamma, \delta, \alpha^{\prime}, \beta^{\prime}, \gamma^{\prime}, \delta^{\prime} \in \mathbb{Q}-\mathbb{Z}$ then

(4) $\mathcal{S}(\alpha, \beta ; \gamma, \delta)=\mathcal{S}\left(\alpha^{\prime}, \beta^{\prime} ; \gamma^{\prime}, \delta^{\prime}\right)\left(\right.$ resp. $\left.\pm \mathcal{S}\left(\alpha^{\prime}, \beta^{\prime} ; \gamma^{\prime}, \delta^{\prime}\right)\right)$ if and only if $(\alpha, \beta$; $\gamma, \delta)$ and $\left(\alpha^{\prime}, \beta^{\prime} ; \gamma^{\prime}, \delta^{\prime}\right)$ are related by a composition of the transformations in (1) and (2) (resp. the transformations in (1), (2) and (3)).

Proof (1) follows from the property of $\mathcal{M}(\alpha, \beta)$ noted earlier.

To prove (2), observe that rotating Figure 2.3 through $180^{\circ}$ about an axis perpendicular to the plane of the paper shows that $\mathcal{S}(\alpha, \beta ; \gamma, \delta)=\mathcal{S}(\delta, \gamma ; \beta, \alpha)$. (A rational tangle is unchanged by rotation through $180^{\circ}$ about any of the three co-ordinate axes.) Also, by Lemma $2.1, \mathcal{S}(\alpha, \beta ; \gamma, \delta)=\mathcal{S}(\beta, \alpha ; \gamma, \delta)$. The group generated by these two permutations is the dihedral group $D_{8}$.

(3) follows by changing all the crossings in the diagrams of $\mathcal{R}(\alpha), \mathcal{R}(\beta), \mathcal{R}(\gamma), \mathcal{R}(\delta)$.

To prove (4), let $K=\mathcal{S}(\alpha, \beta ; \gamma, \delta), K^{\prime}=\mathcal{S}\left(\alpha^{\prime}, \beta^{\prime} ; \gamma^{\prime}, \delta^{\prime}\right)$, and let $M, M^{\prime}$ be the double branched covers of $K$ and $K^{\prime}$ respectively; thus $M=D^{2}(\alpha, \beta) \cup D^{2}(\gamma, \delta)$, and similarly for $M^{\prime}$. Parametrize slopes on the torus $T$, the double branched cover of $S=\partial \mathcal{M}(\alpha, \beta)$, by the parametrization of slopes on $S$ coming from the marking of $\mathcal{M}(\alpha, \beta)$ in Figure 2.4. Thus $1 / 0$ is the slope of the Seifert fiber $\phi$ of $D^{2}(\alpha, \beta), 0 / 1$ is the slope of the Seifert fiber $\psi$ of $D^{2}(\gamma, \delta)$, and similarly for $\phi^{\prime}, \psi^{\prime}$.

Suppose $K=K^{\prime}$. Then there is an orientation-preserving homeomorphism $h: M \rightarrow$ $M^{\prime}$. Since $T$ is, up to orientation-preserving homeomorphism, the unique separating, incompressible torus in $M$, and similarly for $T^{\prime}$, we may suppose that $h(T)=$ 
$T^{\prime}$. We may assume further, by interchanging $\{\alpha, \beta\}$ and $\{\gamma, \delta\}$ if necessary, that $h\left(D^{2}(\alpha, \beta)\right)=D^{2}\left(\alpha^{\prime}, \beta^{\prime}\right)$, and, since the Seifert fiberings of $D^{2}(\alpha, \beta)$ etc. are unique, that $h(\phi)=\phi^{\prime}, h(\psi)=\psi^{\prime}$.

Recall that if $N$ is a Seifert fiber space over $D^{2}$ with two exceptional fibers, then to describe $N$ as $D^{2}(\mu, v)(\mu, v \in \mathbb{Q}-\mathbb{Z})$, we remove disjoint Seifert fibered neighborhoods of the exceptional fibers, getting $P \times S^{1}$, where $P$ is a pair of pants, and choose a section $s: P \rightarrow P \times S^{1}$. In identifying $\widetilde{\mathcal{M}}(\alpha, \beta)$ with $D^{2}(\alpha, \beta)$ we use the section that takes the boundary components of $P$ to curves of slope $0 / 1$ with respect to the markings in Figure 2.4 of $S$ and the boundaries of the rational tangles $\mathcal{R}(\alpha)$ and $\mathcal{R}(\beta)$.

Since $h: D^{2}(\alpha, \beta) \rightarrow D^{2}\left(\alpha^{\prime}, \beta^{\prime}\right)$ is an orientation-preserving homeomorphism which preserves the slopes $1 / 0$ and $0 / 1$ on $S$, the descriptions $D^{2}(\alpha, \beta)$ and $D^{2}\left(\alpha^{\prime}, \beta^{\prime}\right)$ differ only in the possible re-ordering of the two exceptional fibers and the choice of section $s$, subject to $s\left(\partial_{0} P\right)$ having slope $0 / 1$, where $\partial_{0} P$ is the boundary component of $P$ that corresponds to the outer (unfilled) boundary component of $\mathcal{M}(*, *)$ in Figure 2.2. This choice corresponds to twisting a given section along an annulus $a \times S^{1} \subset P \times S^{1}$, where $a$ is an arc in $P$ with one endpoint in each component of $\partial P-\partial_{0} P$. This in turn corresponds to replacing $(\alpha, \beta)$ by $(\alpha+m, \beta-m)$ for some $m \in \mathbb{Z}$. Applying the same considerations to $D^{2}(\gamma, \delta)$ and $D^{2}\left(\gamma^{\prime}, \delta^{\prime}\right)$ gives the desired conclusion.

The parenthetical statement in (4) now follows from (3).

By a crossing move on a knot $K$ we mean the operation of passing one strand of $K$ through another. More precisely, we take a 3-ball $B_{0}$ in $S^{3}$ such that $\left(B_{0}, B_{0} \cap K\right)=\mathcal{T}_{0}$ is a trivial tangle, and replace it by the trivial tangle $\mathcal{T}_{0}^{\prime}$ shown in Figure 2.5. This determines an $\operatorname{arc}(a, \partial a) \subset\left(S^{3}, K\right)$ as shown in Figure 2.5. Note that $\mathcal{T}_{0}$ is a relative regular neighborhood of $(a, \partial a)$ in $\left(S^{3}, K\right)$. Conversely, the $\operatorname{arc} a$, together with a framing of $a$, determines the crossing move. If the resulting knot $K^{\prime}$ is the unknot, we say that $a$ is an unknotting arc for $K$.

Note that we distinguish between a crossing move and a crossing change, reserving the latter term for a change of crossing in a knot diagram.

If $K$ is a knot in $S^{3}$, then $u(K)$ is its unknotting number. That is, $u(K)$ is the smallest number of crossing moves required to unknot $K$.

Write $\mathcal{T}=\left(S^{3}, K\right)-\operatorname{int} \mathcal{T}_{0}$, and assume that $K^{\prime}$ is the unknot. Then taking double branched covers gives

$$
\begin{aligned}
& M=B_{2}(K)=B_{2}(\mathcal{T}) \cup B_{2}\left(\mathcal{T}_{0}\right)=X \cup V_{0}, \\
& S^{3}=B_{2}\left(K^{\prime}\right)=B_{2}(\mathcal{T}) \cup B_{2}\left(\mathcal{T}_{0}^{\prime}\right)=X \cup V_{0}^{\prime},
\end{aligned}
$$

Algebraic 83 Geometric Topology, Volume 6 (2006) 

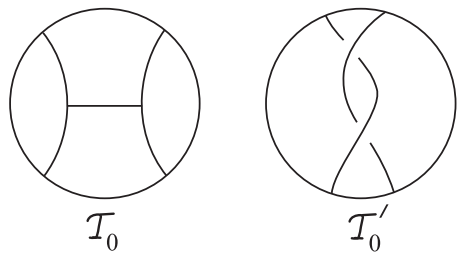

Figure 2.5

where $V_{0}, V_{0}^{\prime}$ are solid tori with meridians $\gamma, \mu$, say, on $\partial X$, such that $\Delta(\gamma, \mu)=2$. Thus the core of $V_{0}^{\prime}$ is a knot $K^{*}$ in $S^{3}$, with exterior $X$ and meridian $\mu$, and $K^{*}(\gamma)=X(\gamma) \cong M$.

This connection between crossing moves and Dehn surgery in the double branched cover is due to Montesinos [24].

We now recall the characteristic toric orbifold decomposition of a knot, due to Bonahon and Siebenmann [3].

Let $K$ be a prime knot in $S^{3}$. Regard $S^{3}$ as an orbifold $O(K)$ with singular set $K$, each point of $K$ having isotropy group rotation of $\mathbb{R}^{3}$ about $\mathbb{R}^{1}$ through angle $\pi$. Since $K$ is prime, the Characteristic Toric Orbifold Splitting Theorem of [3] asserts the existence of a collection $\mathbf{T}(O(K))=\mathbf{T}(K)$ of disjoint incompressible 2-sided toric 2-suborbifolds, unique up to orbifold isotopy, such that (i) each component of $O(K)$ cut along $\mathbf{T}(K)$ is either atoroidal or $S^{1}$-fibered (as an orbifold), and (ii) $\mathbf{T}(K)$ is minimal with respect to this property. (See [3, Splitting Theorem 1].) Each component of $\mathbf{T}(K)$ is either a 2-torus disjoint from $K$ or a Conway sphere.

$\mathbf{T}(K)$ may be described as follows; see Boileau and Zimmermann [2]. Let $M$ be the double branched cover of $\left(S^{3}, K\right)$, with covering involution $h: M \rightarrow M$. Let $\mathbf{T}(M)$ be the JSJ-decomposition of $M$. By Meeks and Scott [20], we may assume that $\mathbf{T}(M)$ is $h$-invariant. For each component $T$ of $\mathbf{T}(M)$ such that $h(T)=T$ and $h$ exchanges the sides of $T$, replace $T$ by two parallel copies that are interchanged by $h$. Denote this new collection of tori by $\mathbf{T}^{+}(M)$. Then $\mathbf{T}(K)$ is the quotient $\mathbf{T}^{+}(M) / h$ in $S^{3}$.

\section{EM-knots and EM-tangles}

In [6] Eudave-Muñoz constructed an infinite family of knots $K=K(\ell, m, n, p)$ such that (1) $K$ has unknotting number 1, (2) $K$ has a (unique) essential Conway sphere $S$, and (3) no unknotting arc for $K$ is disjoint from $S$. Passing to double branched covers these give rise (by [24]; see Section 2) to a family of hyperbolic knots $k(\ell, m, n, p)$ in 
$S^{3}$, called the Eudave-Muñoz knots in [11], each of which has a half-integral toroidal surgery. To distinguish the $K(\ell, m, n, p)$ 's from the $k(\ell, m, n, p)$ 's we shall call the former EM-knots.

Definition The crossing move described in [6] that unknots $K(\ell, m, n, p)$ will be called the standard crossing move of $K(\ell, m, n, p)$.

Recall [6] that the parameters $\ell, m, n, p$ are restricted as follows: one of $n, p$ is always 0 ; $|\ell|>1$; if $p=0$ then $m \neq 0,(\ell, m) \neq(2,1)$ or $(-2,-1)$, and $(m, n) \neq(1,0)$ or $(-1,1)$; if $n=0$ then $m \neq 0$ or 1 , and $(\ell, m, p) \neq(-2,-1,0)$ or $(2,2,1)$.

The EM-knots can be conveniently described in terms of the tangle $\mathcal{S}$ defined in Section 2 (see Figure 2.3 to describe $\mathcal{S}$ as a marked tangle).

Lemma 3.1 The EM-knot $K(\ell, m, n, p)=\mathcal{S}(\alpha, \beta ; \gamma, \delta)$ where $\alpha, \beta, \gamma, \delta$ are as follows:

$$
\begin{aligned}
& p=0: \quad \alpha=-\frac{1}{\ell}, \quad \beta=\frac{m}{\ell m-1}, \quad \gamma=\frac{2 m n+1-m-n}{4 m n-2 m+1}, \quad \delta=-\frac{1}{2} \\
& n=0: \quad \alpha=-\frac{1}{\ell}, \quad \beta=\frac{2 m p-m-p}{\ell(2 m p-m-p)-2 p+1}, \quad \gamma=\frac{m-1}{2 m-1}, \quad \delta=-\frac{1}{2} .
\end{aligned}
$$

Proof This follows immediately from [7, Proposition 5.4] (after allowing for sign errors).

Lemma 3.1, together with the restrictions on the parameters $\ell, m, n, p$, easily implies

Corollary 3.2 Any EM-knot is of the form $\mathcal{S}(\alpha, \beta ; \gamma, \delta)$ with $\alpha, \beta, \gamma, \delta \in \mathbb{Q}-\mathbb{Z}$, $|\alpha|,|\beta|,|\gamma|,|\delta|<1$, and $\alpha \beta<0, \gamma \delta<0$.

We will need to consider a collection of tangles in the 3-ball, the EM-tangles, closely related to the EM-knots. We describe them as two families, corresponding to the cases $p=0$ and $n=0$ of the EM-knots. More precisely, let $\mathcal{A}_{1}(\ell, m)$ be the tangle obtained from the knot $K(\ell, m, n, 0)$ by removing the " $C$ "-tangle, and $\mathcal{A}_{2}(\ell, m)$ be the tangle obtained from $K(\ell, m, 0, p)$ by removing the " $B$ "-tangle. Here $\ell$ and $m$ are subject to the same restrictions as for $K(\ell, m, n, p)$, ie, $|\ell|>1$ in both cases, and for $\mathcal{A}_{1}(\ell, m), m \neq 0,(\ell, m) \neq(2,1)$ or $(-2,-1)$, while for $\mathcal{A}_{2}(\ell, m), m \neq 0$ or 1 .

We therefore have the following: 
Definition 3.3 The EM-tangle $\mathcal{A}_{\varepsilon}(\ell, m)$ is given by $\mathcal{A}_{1}(\ell, m)=\mathcal{S}(\alpha, \beta ; *, \delta)$, $\mathcal{A}_{2}(\ell, m)=\mathcal{S}(\alpha, * ; \gamma, \delta)$, where $\alpha, \beta, \gamma, \delta$ are as follows:

$$
\begin{aligned}
& \varepsilon=1: \alpha=-\frac{1}{\ell}, \quad \beta=\frac{m}{\ell m-1}, \quad \delta=-\frac{1}{2} ;|\ell|>1, m \neq 0, \\
& (\ell, m) \notin\{(2,1),(-2,-1)\} \\
& \varepsilon=2: \alpha=-\frac{1}{\ell}, \quad \gamma=\frac{m-1}{2 m-1}, \quad \delta=-\frac{1}{2} ;|\ell|>1, m \notin\{0,1\} .
\end{aligned}
$$

(The $\mathcal{A}_{\mathcal{E}}(\ell, m)$ are pictured in Figure 4.3 where the twist boxes represent vertical twists.)

$K(\ell, m, n, p)$ contains an essential Conway sphere $S$, decomposing it into two Montesinos tangles: $K(\ell, m, n, p)=\mathcal{M}(\alpha, \beta) \cup_{S} \mathcal{M}(\gamma, \delta)$. This gives rise to a decomposition of the double branched cover of $K(\ell, m, n, p)$ as $N_{1} \cup_{T} N_{2}$, where $N_{i}$ is a Seifert fiber space over the disk with two exceptional fibers, $i=1,2$, and $T=\partial N_{1}=\partial N_{2}=\widetilde{S}$ is the double branched cover of $S$. Similarly, the essential Conway sphere $S$ in $\mathcal{A}_{\varepsilon}(\ell, m)$ gives a decomposition of its double branched cover as $N_{1} \cup_{T} N_{2}$, where $N_{2}$ is as above, and $N_{1}$ is a Seifert fiber space over the annulus with one exceptional fiber.

The remainder of this section is devoted to proving the following theorem which says that the EM-knots and the EM-tangles are determined by their double branched covers.

\section{Theorem 3.4}

(1) Let $K$ be a knot in $S^{3}$ whose double branched cover is homeomorphic to that of $K(\ell, m, n, p)$. Then $K= \pm K(\ell, m, n, p)$.

(2) Let $\mathcal{T}$ be a tangle in $B^{3}$ whose double branched cover is homeomorphic to that of $\mathcal{A}_{\varepsilon}(\ell, m)$. Then $\mathcal{T}$ and $\mathcal{A}_{\varepsilon}(\ell, m)$ are homeomorphic tangles.

In order to prove Theorem 3.4, we first study involutions on the manifolds $D^{2}(*, *)$, $D^{2}(p / q, *)$ and $D^{2}\left(p_{1} / q_{1}, p_{2} / q_{2}\right)$. The definition of equivalence that is appropriate to our purposes is the following. Two homeomorphisms $f, g: X \rightarrow X$ are strongly conjugate if there is a homeomorphism $h: X \rightarrow X$ isotopic to the identity such that $f=h^{-1} g h$. If $X_{0} \subset X$, then $f$ and $g$ are strongly conjugate rel $X_{0}$ if $h$ can be chosen to be isotopic to the identity by an isotopy fixed on $X_{0}$. The set of fixed points of an involution $\tau$ will be denoted by $\operatorname{Fix}(\tau)$.

To define a standard model for a pair of pants $P$, let $D^{2}$ be the unit disk in $\mathbb{R}^{2}$, let $D_{1}$ and $D_{2}$ be disjoint round disks in int $D^{2}$ with their centers on the $x$-axis, and let $P=D^{2}-\operatorname{int}\left(D_{1} \cup D_{2}\right)$. The map $(x, y) \mapsto(x,-y)$ defines an orientation-reversing involution $\rho_{P}$ of $P$, which we will call reflection. 
Lemma 3.5 Let $\tau$ be a non-trivial involution on a pair of pants $P$. If each boundary component of $P$ is invariant under $\tau$ then $\tau$ is strongly conjugate to reflection.

Proof Let $\alpha=P \cap(x$-axis $)$, a disjoint union of three arcs properly embedded in $P$. Since the restriction of $\tau$ to each boundary component of $P$ is either the identity, conjugate to rotation through $\pi$, or conjugate to reflection, we may assume that $\partial \alpha$ is invariant under $\tau$. By analyzing the intersections of $\alpha$ and $\tau(\alpha)$, one can show that after conjugating $\tau, \alpha$ can be taken to be invariant under $\tau$. This implies that $\alpha$ is fixed by $\tau$. The two disks of $P-\alpha$ are either exchanged or invariant. In the first case, $\tau$ is conjugate to reflection, and in the second $\tau$ must be the identity. Finally, since any homeomorphism of $P$ is isotopic to one that commutes with $\rho_{P}$, in the first case $\tau$ is strongly conjugate to $\rho_{P}$.

Recall (Section 2) that $D^{2}(*, *)$ (resp. $D^{2}(p / q, *)$, resp. $\left.D^{2}\left(p_{1} / q_{1}, p_{2} / q_{2}\right)\right)$ is the double branched cover of the tangle $\mathcal{M}(*, *)$ (resp. $\mathcal{M}(p / q, *)$, resp. $\mathcal{M}\left(p_{1} / q_{1}\right.$, $\left.\left.p_{2} / q_{2}\right)\right)$. The standard involution on $D^{2}(*, *), D^{2}(p / q, *)$ or $D^{2}\left(p_{1} / q_{1}, p_{2} / q_{2}\right)$ is the non-trivial covering transformation corresponding to this double branched cover. Note that in particular, identifying $D^{2}(*, *)$ with $P \times S^{1}$, the standard involution on $D^{2}(*, *)$ is the map $(x, \theta) \mapsto\left(\rho_{P}(x),-\theta\right)$.

Lemma 3.6 Let $\tau$ be a non-trivial orientation-preserving involution on $P \times S^{1}$. If each component of the boundary is invariant under $\tau$ then $\tau$ is strongly conjugate to either the standard involution or a free involution that leaves each $S^{1}$-fiber invariant.

Proof By Tollefson [35], there is a Seifert fibration of $P \times S^{1}$ that is invariant under $\tau$. As the Seifert fibration is unique up to isotopy, we may therefore assume, after strongly conjugating $\tau$, that $\tau$ preserves the product $S^{1}$-fibration of $P \times S^{1}$. Thus $\tau$ induces an involution $\tau_{P}$ on $P$. By Lemma 3.5, $\tau_{P}$ is either the identity or strongly conjugate to $\rho_{\boldsymbol{P}}$.

If $\tau_{P}$ is the identity, then $\tau$ takes each $S^{1}$-fiber to itself by an orientation-preserving involution, hence by either the identity or a map conjugate to rotation through $\pi$. By continuity, the action is the same on each fiber. Therefore $\tau$ is either the identity or free.

We may suppose, then, that $\tau_{P}$ is strongly conjugate to $\rho_{P}$, and hence, by strongly conjugating $\tau$, that $\tau_{P}=\rho_{P}$.

Let $C$ be a boundary component of $P$. Note that $\tau_{P}$ fixes two points in $C$. The restriction of $\tau$ to each of the two corresponding $S^{1}$-fibers is therefore conjugate to reflection $\theta \mapsto-\theta$. It follows that the restriction of $\tau$ to $C \times S^{1}$ is strongly conjugate 
to $-I$, given by $(\varphi, \theta) \mapsto(-\varphi,-\theta)$ (see Hartley [13]). So we may assume that $\tau=-I$ on each boundary component of $P \times S^{1}$. Since any two $S^{1}$-fibrations of $P \times S^{1}$ that agree on the boundary are isotopic rel $\partial$, we can still assume that $\tau$ preserves the product fibration and that $\tau_{P}=\rho_{P}$.

Let $\alpha_{1}, \alpha_{2}$ be the two arc components of $\operatorname{Fix}\left(\rho_{P}\right)$ shown in Figure 3.1, and let $C_{1}, C_{2}$ be the two boundary components of $P$ indicated in the same figure.

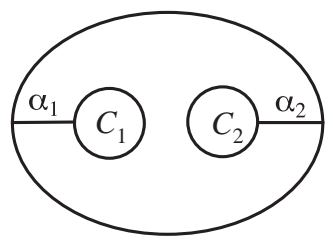

Figure 3.1

Let $A_{i}$ be the vertical annulus $\alpha_{i} \times S^{1}$, and let $T_{i}$ be the boundary torus $C_{i} \times S^{1}$, $i=1,2$. Note that $A_{i}$ is invariant under $\tau, i=1,2$. Then $\tau \mid\left(A_{i}=\alpha_{i} \times S^{1}\right)$ is conjugate to the involution $(x, \theta) \mapsto(x,-\theta)$, by a homeomorphism $g_{i}: A_{i} \rightarrow A_{i}$ that is isotopic rel $\partial$ to a power of a Dehn twist along the core of $A_{i}$. Hence, conjugating $\tau$ by the corresponding power of a vertical Dehn twist $h_{i}$ along a torus in a collar neighborhood of $T_{i}$, we may assume that $\tau \mid A_{i}$ is $(x, \theta) \mapsto(x,-\theta)$. Since $h_{i}$ is isotopic to the identity, the strong conjugacy class of $\tau$ is unchanged.

By a further isotopy rel $\partial$, we may assume that $A_{i}=A_{i} \times\{0\}$ has a neighborhood $A_{i} \times[-1,1]$ on which $\tau$ acts by $(x, \theta, t) \mapsto(x,-\theta,-t)$. Removing $\left(A_{1} \cup A_{2}\right) \times(-1,1)$ from $P \times S^{1}, \tau$ induces an involution $\tau_{0}$ on $D^{2} \times S^{1}$ which is equal to $-I$ on the boundary. Hence $\tau_{0}$ is strongly conjugate rel $\partial$ to the involution $((x, y), \theta) \mapsto$ $((x,-y),-\theta)$ [13]. Reattaching $\left(A_{1} \cup A_{2}\right) \times[-1,1]$ we get that $\tau$ is strongly conjugate to the standard involution.

Lemma 3.7 Let $\tau$ be a non-trivial orientation-preserving involution on $D^{2}\left(p_{1} / q_{1}, p_{2} / q_{2}\right)$, where $q_{1} \neq q_{2}$.

(1) If $\operatorname{Fix}(\tau)$ has non-empty intersection with the boundary then $\tau$ is strongly conjugate to the standard involution.

(2) If $\tau$ acts freely on the boundary then the Seifert fibration of $D^{2}\left(p_{1} / q_{1}, p_{2} / q_{2}\right)$ may be isotoped so that $\tau$ leaves each Seifert fiber on the boundary invariant.

Proof By [35], $D^{2}\left(p_{1} / q_{1}, p_{2} / q_{2}\right)$ has a Seifert fibration for which $\tau$ is fiber-preserving. Since the Seifert fibration is unique up to isotopy, and since $q_{1} \neq q_{2}$, the 
exceptional fibers must be invariant, and hence they have disjoint invariant fibered neighborhoods $V_{1}$ and $V_{2}$, say. Thus $\tau$ restricts to an involution $\tau_{0}$ on the complement of these neighborhoods, $D^{2}(*, *)=P \times S^{1}$. Note that $\tau_{0}$ leaves each boundary component of $D^{2}(*, *)$ invariant.

(1) Here $\operatorname{Fix}\left(\tau_{0}\right) \neq \varnothing$, so by Lemma $3.6 \tau_{0}$ is strongly conjugate to the standard involution. Extending over $V_{1}$ and $V_{2}$, and using [13], we get that $\tau$ is strongly conjugate to the standard involution.

(2) Since $\tau_{0}$ acts freely on at least one of the boundary components of $D^{2}(*, *)$, by Lemma 3.6 the (product) Seifert fibration of $D^{2}(*, *)$ may be isotoped so that $\tau$ leaves each fiber invariant. Now $\tau \mid \partial V_{i}$ can be extended to an involution $\tau_{i}$ of $V_{i}$ that leaves each Seifert fiber invariant. Since $\tau_{i}$ and $\tau \mid V_{i}$ agree on $\partial V_{i}$, they are strongly conjugate rel $\partial V_{i}$ [13]. The corresponding isotopy of $V_{i}\left(\operatorname{rel} \partial V_{i}\right)$ takes the Seifert fibration of $V_{i}$ to one such that each fiber is invariant under $\tau$.

Lemma 3.8 Let $\tau$ be a non-trivial orientation-preserving involution on $D^{2}(p / q, *)$ such that $\operatorname{Fix}(\tau)$ has non-empty intersection with the boundary. Then $\tau$ is strongly conjugate to the standard involution.

Proof This is the same as the proof of Part (1) of Lemma 3.7.

Proof of Theorem 3.4 Case (1) Write $K_{0}=K(\ell, m, n, p)$. Then $\left(S^{3}, K_{0}\right)=$ $\left(B_{1}, A_{1}\right) \cup_{S}\left(B_{2}, A_{2}\right)$, where $S$ is an essential Conway sphere and $\left(B_{i}, A_{i}\right)$ is a Montesinos tangle of length $2, i=1,2$. The double branched cover of $\left(S^{3}, K_{0}\right)$ is $N=N_{1} \cup \widetilde{S} N_{2}$, where $N_{i}$, the double branched cover of $\left(B_{i}, A_{i}\right)$, is a Seifert fiber space over the disk with two exceptional fibers, $i=1,2$, and $\widetilde{S}=\partial N_{1}=\partial N_{2}$ is the double branched cover of $\left(S, S \cap K_{0}\right)$. The Seifert fibers of $N_{1}$ and $N_{2}$ intersect once on $\widetilde{S}$. The covering involution $\sigma: N \rightarrow N$ restricts to the standard involution $\sigma_{i}$ on $N_{i}, i=1,2$.

Now suppose $K$ is a knot in $S^{3}$ whose double branched cover is homeomorphic to $N$. Let $\tau: N \rightarrow N$ be the corresponding covering involution. Since $\widetilde{S}$ is the unique incompressible torus in $N$, up to isotopy, by [20, Theorem 8.6] we may assume that $\widetilde{S}$ is invariant under $\tau$.

Claim 1 Each $N_{i}$ is invariant under $\tau$.

Proof If $\tau$ interchanges $N_{1}$ and $N_{2}$ then Fix $(\tau)$ is contained in $\widetilde{S}$. With respect to some parametrization of $\widetilde{S}$ as $S^{1} \times S^{1}$, Fix $(\tau)$ is a $(2,1)$-curve and $\tau$ leaves each $(0,1)$-curve $\gamma$ invariant, taking it to itself by reflection in the pair of points $\operatorname{Fix}(\tau) \cap \gamma$ 
(thus the quotient $\widetilde{S} / \tau$ is a Möbius band). Then $S^{3}=N_{1} /(\tau \mid \widetilde{S})$ is homeomorphic to $N_{1}$ with a solid torus $V$ attached so that $\gamma$ bounds a meridian disk of $V$. Hence $N_{1}$ is a knot exterior with meridian $\gamma$. Applying the same argument to $N_{2}$, we see that in $N=N_{1} \cup N_{2}$ the meridians of $N_{1}$ and $N_{2}$ are identified. But this is not true: when each side of $N$ is the exterior of a knot in $S^{3}$, the argument in Lemma 1.3 of [6] (or Lemmas 3.1 and 9.5) shows that the meridian of one side is identified with the Seifert fiber of the other.

Let $\tau_{i}$ be the restriction of $\tau$ to $N_{i}, i=1,2$.

Claim $2 \tau_{i}$ is strongly conjugate to the standard involution $\sigma_{i}$ on $N_{i}, i=1,2$.

Proof If Fix $(\tau)$ meets $\widetilde{S}$ then the result follows from Lemma 3.7(1) and Lemma 3.9 below.

If Fix $(\tau)$ is disjoint from $\widetilde{S}$, then by Lemma 3.7(2) and Lemma 3.9 the Seifert fibrations of $N_{1}$ and $N_{2}$ can be isotoped so that, on $\widetilde{S}$, each $S^{1}$-fiber of each fibration is invariant under $\tau$. But since the fibers of the two fibrations intersect once on $\widetilde{S}$, this is clearly impossible.

Write $S_{i}=\partial B_{i}, i=1,2$, and let $f:\left(S_{1}, S_{1} \cap A_{1}\right) \rightarrow\left(S_{2}, S_{2} \cap A_{2}\right)$ be the gluing homeomorphism that defines $\left(S^{3}, K_{0}\right)=\left(B_{1}, A_{1}\right) \cup_{f}\left(B_{2}, A_{2}\right)$. To compare $K_{0}$ and $K$, we need the notion of a mutation involution, which is defined at the beginning of Section 7.

Claim $3\left(S^{3}, K\right)$ is homeomorphic to $\left(B_{1}, A_{1}\right) \cup_{\mu f}\left(B_{2}, A_{2}\right)$ for some mutation involution $\mu$ of $\left(S_{2}, S_{2} \cap A_{2}\right)$.

Proof Let $\tilde{f}: \partial N_{1} \rightarrow \partial N_{2}$ be a lift of $f$, giving $N=N_{1} \cup \tilde{f} N_{2}$. Note that $\tilde{f} \sigma_{1}=\sigma_{2} \tilde{f}$ and $\tilde{f} \tau_{1}=\tau_{2} \tilde{f}$. Also, $N / \tau=\left(N_{1} / \tau_{1}\right) \cup g\left(N_{2} / \tau_{2}\right)$ for some $g: \partial\left(N_{1} / \tau_{1}\right) \rightarrow \partial\left(N_{2} / \tau_{2}\right)$ such that $\tilde{f}$ is a lift of $g$.

By Claim 2, there is a homeomorphism $\tilde{h}_{i}: N_{i} \rightarrow N_{i}$, isotopic to the identity, such that $\tau_{i}=\widetilde{h}_{i}^{-1} \sigma_{i} \widetilde{h}_{i}, i=1,2$. Then $\widetilde{h}_{i}$ induces a homeomorphism $h_{i}: N_{i} / \tau_{i} \rightarrow N_{i} / \sigma_{i}=$ $\left(B_{i}, A_{i}\right), i=1,2$. Let $\partial h_{i}$ be the restriction of $h_{i}$ to $\partial\left(N_{i} / \tau_{i}\right)$. Then $h_{1} \cup h_{2}$ induces a homeomorphism $h: N / \tau=\left(N_{1} / \tau_{1}\right) \cup_{g}\left(N_{2} / \tau_{2}\right) \rightarrow\left(B_{1}, A_{1}\right) \cup_{e}\left(B_{2}, A_{2}\right)$, where $e=\left(\partial h_{2}\right)^{-1} g\left(\partial h_{1}\right)$. Then $e$ lifts to $\widetilde{e}=\left(\partial \tilde{h}_{2}\right)^{-1} \tilde{f}\left(\partial \tilde{h}_{1}\right)$, which is isotopic to $\tilde{f}$.

Let $\mu$ be the mutation involution of $\left(S_{2}, S_{2} \cap A_{2}\right)$ such that the composition $\mu f$ agrees with $e$ on some point of $S_{1} \cap A_{1}$. Since $\widetilde{e}$ is isotopic to $\tilde{f}, e$ and $f$ induce the same function from the set of (unoriented) isotopy classes of essential simple closed 
curves in $S_{1}-\left(S_{1} \cap A_{1}\right)$ to the set of those in $S_{2}-\left(S_{2} \cap A_{2}\right)$. Hence $e$ and $\mu f$ do also. Since $e$ and $\mu f$ agree on a point of $S_{1} \cap A_{1}$, they must be isotopic as maps of pairs. Therefore $\left(S^{3}, K\right) \cong\left(B_{1}, A_{1}\right) \cup_{e}\left(B_{2}, A_{2}\right) \cong\left(B_{1}, A_{1}\right) \cup_{\mu f}\left(B_{2}, A_{2}\right)$.

By Claim 3, $K$ is a mutation of $K_{0}$ along $S$. By [6] such mutations yield $K_{0}$ again. This completes the proof of the theorem in Case (1).

Case (2) We have $\mathcal{A}_{\varepsilon}(\ell, m)=\left(B_{1}, A_{1}\right) \cup_{S}\left(B_{2}, A_{2}\right)$, where $B_{1}$ is $S^{2} \times I,\left(B_{1}, A_{1}\right)=$ $\mathcal{M}(p / q, *)$, and $\left(B_{2}, A_{2}\right)$ is a Montesinos tangle of length 2 as in Case (1); see Definition 3.3. The double branched cover of $\mathcal{A}_{\varepsilon}(\ell, m)$ is then $N=N_{1} \cup \widetilde{S} N_{2}$, where $N_{1}$ is now a Seifert fiber space over the annulus with one exceptional fiber. The covering involution $\sigma$ restricts to the standard involution $\sigma_{i}$ on $N_{i}, i=1,2$.

Suppose $\mathcal{T}$ is a tangle in $B^{3}$ whose double branched cover is homeomorphic to $N$, and let $\tau$ be the corresponding covering involution. Since $\widetilde{S}$ is the unique essential torus in $N$, we may assume $\tau(\widetilde{S})=\widetilde{S}$. Let $\tau_{i}$ be the restriction of $\tau$ to $N_{i}, i=1,2$. By Lemma 3.8, $\tau_{1}$ is strongly conjugate to the standard involution on $N_{1}$. In particular $\operatorname{Fix}(\tau) \cap \widetilde{S} \neq \varnothing$, and so, by Lemmas 3.7(1) and 3.9, $\tau_{2}$ is strongly conjugate to the standard involution on $N_{2}$. Now Claim 3 holds, exactly as in Case (1). Since $\mathcal{A}_{\varepsilon}(\ell, m)$ is unchanged by mutation along $S$, (by Lemma 2.1 and the fact that rotating a rational tangle through $\pi$ about a co-ordinate axis does not change it), $\mathcal{T}$ is homeomorphic to $\mathcal{A}_{\varepsilon}(\ell, m)$.

Lemma 3.9 Let $N=N_{1} \cup N_{2}$ be the double branched cover of $S^{3}$ over $K(\ell, m, n, p)$ or $\mathcal{A}_{\varepsilon}(\ell, m)$, as in the discussion before the statement of Theorem 3.4. Let $q_{1}, q_{2}$ be the orders of the two exceptional fibers of $N_{i}$ for some $i$. Then $q_{1} \neq q_{2}$.

Proof When $N$ is the cover of an EM-tangle, the lemma applies to $N_{2}$. By Definition 3.3 the exceptional fibers are of orders $(|\ell|,|1-\ell m|)$ with $|\ell|>1, m \neq 0$ or of orders $(2,|2 m-1|)$ where $m \neq 0,1$. In either case, the lemma easily follows.

So assume $N$ is the double branched cover of $K(\ell, m, n, p)$, and recall Lemma 3.1.

If $p=0$, the orders of the exceptional fibers are $(|\ell|,|1-\ell m|)$ for $N_{1}$ and $(2, \mid 4 m n-$ $2 m+1 \mid)$ for $N_{2}$. In this case the lemma is clear.

So we assume $n=0$. In this case the exceptional fibers have orders $(2,|2 m-1|)$ in $N_{1}$ and $(|\ell|,|2 \ell m p-\ell m-\ell p-2 p+1|)$ in $N_{2}$. We need to consider the solutions to

$$
\begin{array}{rlrl} 
\pm l & =2 \ell m p-\ell m-\ell p-2 p+1 \\
& \Longleftrightarrow & 2 p-1 & =\ell(2 m p-m-p \pm 1) \\
& \Longleftrightarrow \quad 2(2 p-1) & =\ell(4 m p-2 m-2 p \pm 2) \\
& \Longleftrightarrow \quad 2(2 p-1) & =\ell((2 m-1)(2 p-1)+a)
\end{array}
$$

Algebraic $\&$ Geometric Topology, Volume 6 (2006) 
where $a$ is -3 or 1 . When $n=0$, we assume that $m \neq 0,1$. Thus $|2 m-1| \geq 3$. Then

$$
|(2 m-1)(2 p-1)+a| \geq|(2 m-1)(2 p-1)|-3 \geq 2|2 p-1|+|2 p-1|-3 .
$$

Since $|\ell| \geq 2$, (1) implies that $|2 p-1| \geq 3$. Thus (2) becomes

$$
|2(2 p-1)| \geq|\ell||2(2 p-1)|
$$

a contradiction.

\section{Knots in solid tori}

In this section we describe the hyperbolic knots in a solid torus that have a non-integral toroidal Dehn surgery. We gave a description of such knots in [11, Corollary A.2]; here we sharpen this to a complete characterization.

The exteriors of the knots are the double branched covers of certain tangles in $S^{2} \times I$, which we now describe. Let $\mathcal{C}(A, B, C, D)$ be the tangle shown in Figure 4.1. (To

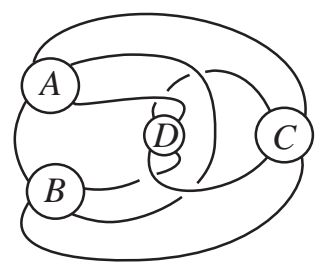

Figure 4.1: $\mathcal{C}(A, B, C, D)$

be consistent with the notation of [7], we here regard $A, B, C, D$ as denoting rational tangles; a puncture that is not filled in will as usual be indicated by a $*$.) Note that in the terminology of [11], $\mathcal{C}(A, B, C, D)=\mathcal{B}(A, B, C)+R(D)=\mathcal{P}\left(A, B, C, \frac{1}{2}, D\right)$. Define tangles $\mathcal{T}_{1}(\ell, m)$ and $\mathcal{T}_{2}(\ell, m)$ in $S^{2} \times I$ as follows.

$\mathcal{T}_{1}(\ell, m)=\mathcal{C}(A, B, *, *)$, where $A=\mathcal{R}(\ell), B=\mathcal{R}(m,-\ell)$, and $\ell, m$ are integers such that $|\ell|>1, m \neq 0$, and $(\ell, m) \neq(2,1)$ or $(-2,-1)$.

$\mathcal{T}_{2}(\ell, m)=\mathcal{C}(A, *, C, *)$, where $A=\mathcal{R}(\ell), C=\mathcal{R}(m-1,2,0)$, and $\ell, m$ are integers such that $|\ell|>1, m \neq 0,1$.

See Figure 4.2 (the boxes correspond to vertical twists).

Note that $\mathcal{T}_{1}(\ell, m)$ is obtained by removing the " $C$-tangle" from $B(\ell, m, n, 0)$ of [6], and similarly $\mathcal{T}_{2}(\ell, m)$ is obtained by removing the " $B$-tangle" from $B(\ell, m, 0, p)$. 


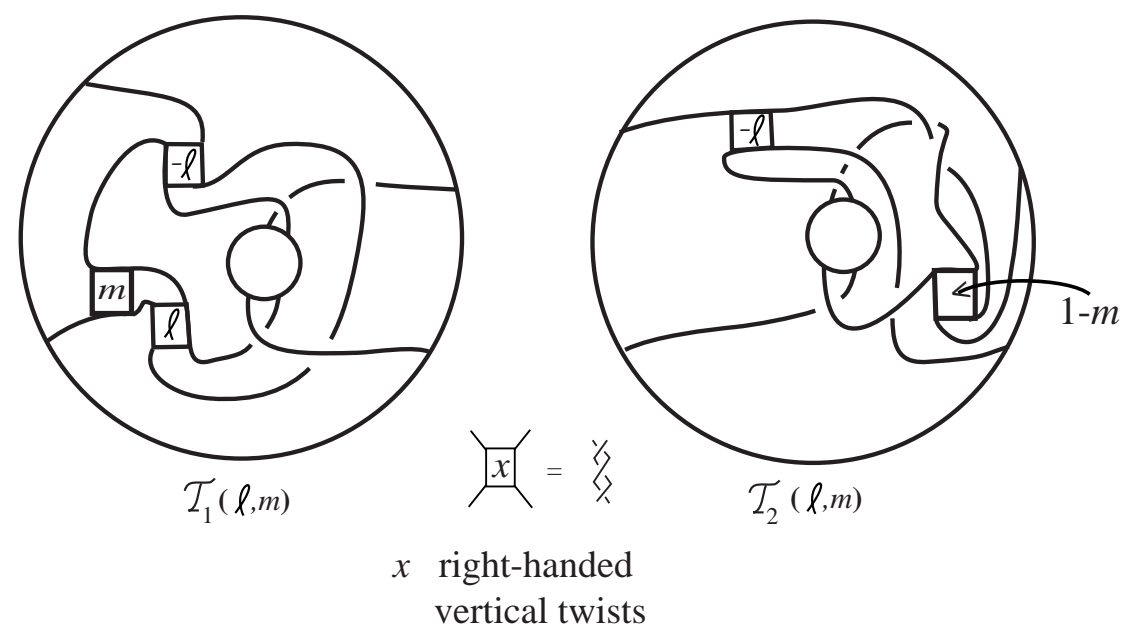

Figure 4.2

Filling the " $D$ "-puncture of $\mathcal{T}_{1}(\ell, m)$ or $\mathcal{T}_{2}(\ell, m)$ with the $\frac{1}{0}$-tangle, ie, $\mathcal{C}(A, B$, $\left.*, \frac{1}{0}\right)$ or $\mathcal{C}\left(A, *, C, \frac{1}{0}\right)$ with $A, B, C$ as indicated above, gives a rational tangle. So, the corresponding $\frac{1}{0}$-Dehn filling of the double branched cover of $\mathcal{T}_{\mathcal{E}}(\ell, m), \varepsilon \in\{1,2\}$, is a solid torus. Let $J_{\varepsilon}(\ell, m)$ denote the core of this Dehn filling, seen as a knot in this solid torus.

Note that the EM-tangle $\mathcal{A}_{\varepsilon}(\ell, m), \varepsilon=1,2$, defined in Section 3, is the tangle in $B^{3}$ obtained by filling the " $D$ "-puncture of $\mathcal{T}_{\varepsilon}(\ell, m)$ with the $\frac{1}{2}$-tangle. That is

$$
\begin{aligned}
& \mathcal{A}_{1}(\ell, m)=\mathcal{C}\left(A, B, *, \frac{1}{2}\right) \\
& \mathcal{A}_{2}(\ell, m)=\mathcal{C}\left(A, *, C, \frac{1}{2}\right)
\end{aligned}
$$

(with $A, B$ as for $\mathcal{T}_{1}(\ell, m), \mathcal{T}_{2}(\ell, m)$ ). See Figure 4.3, which gives a marking to $\mathcal{A}_{\varepsilon}(\ell, m)$.

Definition Denote by $\mathcal{T}_{\varepsilon}(\ell, m)\left(\frac{p}{q}\right)$ the tangle in the 3-ball gotten by filling the $D$-puncture of $\mathcal{T}_{\ell}(\ell, m)$ with the rational tangle $\mathcal{R}(p / q)$. The change in filling $\mathcal{T}_{\varepsilon}(\ell, m)\left(\frac{1}{2}\right)$ to $\mathcal{T}(\ell, m)\left(\frac{1}{0}\right)$ corresponds to a crossing move taking the toroidal tangle $\mathcal{A}_{\varepsilon}(\ell, m)$ to a rational tangle. We will refer to this as the standard crossing move on $\mathcal{A}_{\varepsilon}(\ell, m)$. The arc guiding this crossing change is the standard unknotting arc for $\mathcal{A}_{\varepsilon}(\ell, m)$.

$\mathcal{A}_{\varepsilon}(\ell, m) \quad(\varepsilon=1$ or 2$)$ contains an essential Conway sphere $S$, which induces a decomposition of the double branched covering $\widetilde{\mathcal{A}}_{\varepsilon}(\ell, m)=N_{1} \cup_{T} M_{2}$, where $N_{1}$ 
is a Seifert fiber space over $A^{2}$ with one exceptional fiber, and $M_{2}$ is a Seifert fiber space over $D^{2}$ with two exceptional fibers. See Figure 4.3. Thus if $J=J_{\varepsilon}(\ell, m)$ for some $\varepsilon, \ell, m$, then $J$ is a knot in $S^{1} \times D^{2}$, with meridian $\mu$, say, and $J(\gamma)=$ $\widetilde{\mathcal{A}}_{\varepsilon}(\ell, m)=N_{1} \cup_{T} M_{2}$ contains an essential separating torus $T$, for some $\gamma$ such that $\Delta(\gamma, \mu)=2$.

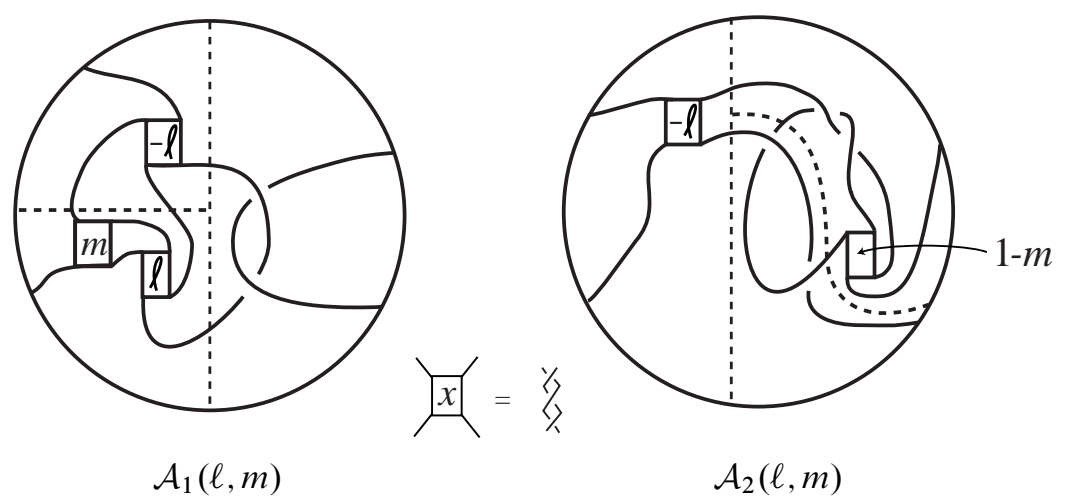

Figure 4.3

Theorem 4.1 $J_{\varepsilon}(\ell, m)$ is a hyperbolic knot in the solid torus.

We give the proof of Theorem 4.1 at the end of this section.

The following theorem says the $J_{\varepsilon}(\ell, m)$ are exactly the hyperbolic knots in solid tori which admit non-integral toroidal surgeries.

Theorem 4.2 Let $J$ be a knot in a solid torus whose exterior is irreducible and atoroidal. Let $\mu$ be the meridian of $J$ and suppose that $J(\gamma)$ contains an essential torus for some $\gamma$ with $\Delta(\gamma, \mu) \geq 2$. Then $\Delta(\gamma, \mu)=2$ and $J=J_{\varepsilon}(\ell, m)$ for some $\varepsilon, \ell, m$.

Remark $J=J_{\varepsilon}(\ell, m)$ means there is a homeomorphism of the solid torus (possibly orientation-reversing) taking $J$ to $J_{\varepsilon}(\ell, m)$.

The following is the corresponding statement about tangles. Theorem 3.4(2) says that $\mathcal{A}_{\varepsilon}(\ell, m)$ is determined by its double branched cover. At this point, it is not known if the same is true for $\mathcal{T}_{\varepsilon}(\ell, m)$. This complicates the statement of Theorem 4.3. 
Theorem 4.3 Let $\mathcal{T}(*, *)$ be a tangle in $S^{2} \times I$ which is irreducible and atoroidal as a $\mathbb{Z}_{2}$-orbifold. If $\mathcal{T}(*, \alpha)$ is rational, and $\mathcal{T}(*, \beta)$ is orbifold-toroidal, where $\Delta(\alpha, \beta) \geq 2$, then the double branched cover of $\mathcal{T}(*, *)$ is homeomorphic to the double branched cover of $\mathcal{T}_{\varepsilon}(\ell, m)$ for some $\varepsilon, \ell, m$. Under this homeomorphism, the slopes $\alpha, \beta$ on $\mathcal{T}$ correspond to slopes $1 / 0,1 / 2$ respectively on $D$ of $\mathcal{C}$.

Furthermore, there are tangle homeomorphisms $h_{1}: \mathcal{T}(*, \beta) \rightarrow \mathcal{A}_{\varepsilon}(\ell, m)=$ $\mathcal{T}_{\varepsilon}(\ell, m)\left(\frac{1}{2}\right)$ and $h_{2}: \mathcal{T}(*, \alpha) \rightarrow \mathcal{T}_{\varepsilon}(\ell, m)\left(\frac{1}{0}\right)$ such that $\left(h_{2} \mid \partial\right)\left(h_{1} \mid \partial\right)^{-1}$ is the identity (where $\partial \mathcal{T}(*, \beta)=\partial \mathcal{T}(*, \alpha) \subset \partial \mathcal{T}(*, *)$, and $\mathcal{T}_{\varepsilon}(\ell, m)\left(\frac{1}{0}\right), \mathcal{T}_{\varepsilon}(\ell, m)\left(\frac{1}{2}\right)$ are marked from Figure 4.2).

Addendum $\mathcal{T}(*, \alpha)$ is rational and thus determines a slope, $\frac{p_{1}}{q_{1}}$, on its boundary. $\mathcal{T}(*, \beta)$ is the tangle $\mathcal{T}_{\varepsilon}(\ell, m)$ whose double branched cover contains a unique essential annulus. The boundary of this annulus determines a slope on the boundary of the cover, which determines a tangle slope, $\frac{p_{2}}{q_{2}}$, on the boundary of $\mathcal{T}(*, \beta)$. Then $\Delta\left(p_{1} / q_{1}, p_{2} / q_{2}\right)=\left|p_{1} q_{2}-p_{2} q_{1}\right|>1$.

Proof of Theorem 4.3 Let $\mathcal{T}(*, *)$ be as in the theorem. Let $X$ be its double branched cover. Let $X(\alpha), X(\beta)$ be the Dehn fillings of $X$ corresponding to the double branched covers of $\mathcal{T}(*, \alpha), \mathcal{T}(*, \beta)$ (resp.). Then by assumption, $X$ is irreducible and atoroidal, $X(\alpha)$ is a solid torus and $X(\beta)$ is toroidal. Since $\Delta(\alpha, \beta)>1$, Corollary A.2 of [11] proves that $X$ is the double branched cover of $\mathcal{C}(A, B, C, *)$ where one of $A, B, C$ is the empty tangle and the others are rational (using the fact proven there that $\left.\frac{1}{2} \in\left\{\alpha^{\prime}, \beta^{\prime}, \gamma^{\prime}\right\}\right)$. Furthermore, under this identification, $\alpha=\frac{1}{0}, \beta=\frac{1}{2}$. That is, $\mathcal{C}\left(A, B, C, \frac{1}{0}\right)$ is a rational tangle, and $\mathcal{C}\left(A, B, C, \frac{1}{2}\right)$ is orbifold-toroidal. By symmetry we may assume that either $B$ or $C$ is the empty tangle above. Lemma 4.4 and Lemma 4.5 below show that $A, B, C$ are as in the definition of $\mathcal{T}_{\varepsilon}(\ell, m)$. Now the double branched cover of $\mathcal{T}(*, \beta)$ is the same as the double branched cover of $\mathcal{C}\left(A, B, C, \frac{1}{2}\right)=\mathcal{A}_{\varepsilon}(\ell, m)$. By Theorem 3.4, $\mathcal{T}(*, \beta)$ and $\mathcal{A}_{\varepsilon}(\ell, m)$ are homeomorphic tangles. Such a homeomorphism $h_{1}$ determines a framing on $\partial X(\beta)\left(\mathcal{A}_{\varepsilon}(\ell, m)\right.$ is a marked tangle), hence on $\partial X(\alpha)$. The meridian disk of $X(\alpha)$ determines a rational number in this framing which corresponds to the rational tangle $\mathcal{T}_{\varepsilon}(\ell, m)\left(\frac{1}{0}\right)$. This implies that $\mathcal{T}(*, \alpha)$ is the same as the rational tangle $\mathcal{T}_{\mathcal{E}}(\ell, m)\left(\frac{1}{0}\right)$ under the marking determined by $h_{1}$. This is the second paragraph of Theorem 4.3.

Proof of Addendum to Theorem 4.3 In the context of the proof of Theorem 4.3, $X(\alpha), X(\beta)$ are the double branched covers of $\mathcal{C}\left(A, B, C, \frac{1}{0}\right), \mathcal{C}\left(A, B, C, \frac{1}{2}\right)$ with $A, B, C$ as in the definition of $\mathcal{T}_{\varepsilon}(\ell, m)$. The slope of the essential annulus of $X(\beta)$ corresponds to the tangle slope $\frac{1}{0}$ in Figure 4.2 (see Figure 4.3). The slope corresponding 
to the rational tangle $\mathcal{T}(*, \alpha)$ corresponds to the slope of the meridian disk in $X(\alpha)$. This in turn corresponds in Figure 4.2 to the $\frac{p}{q}$ of the rational tangle $\mathcal{C}\left(A, B, C, \frac{1}{0}\right)$. We need to show then that $|q|>1$. For $\mathcal{T}_{1}(\ell, m)$, we see this by simply noting that $\mathcal{C}\left(A, B, C, \frac{1}{0}\right)$ capped off by strands of slope $\frac{1}{0}$ is the unlink only when $m=0$. For $\mathcal{T}_{2}(\ell, m), \mathcal{C}\left(A, *, C, \frac{1}{0}\right)$ corresponds to the rational number $\frac{2 m-1}{\ell(2 m-1)-2}$. That is, $q=\ell(2 m-1)-2$. The conditions that $|\ell|>1, m \neq 0,1$ imply that $|q|>1$.

Proof of Theorem 4.2 This is the proof of Theorem 4.3 using the definition of $J_{\varepsilon}(\ell, m)$, and without Theorem 3.4.

Lemma 4.4 If $\mathcal{C}\left(A, *, C, \frac{1}{0}\right)$ is a rational tangle and $\mathcal{C}\left(A, *, C, \frac{1}{2}\right)$ is orbifold-toroidal, then $A=\mathcal{R}(s), C=\mathcal{R}(t, 2,0)$ for $s, t \in \mathbb{Z}$ with $|s|,|2 t+1|>1$.

Proof We follow the argument of Lemma 5.1 of [7]. Rewrite $\mathcal{C}\left(A, *, C, \frac{1}{0}\right)$ as in Figure 4.4. From this we deduce that $C^{\prime}=\mathcal{R}\left(\frac{1}{t^{\prime}}\right), t^{\prime} \in \mathbb{Z}$. Thus $\mathcal{C}\left(A, *, C, \frac{1}{0}\right)$ is as in Figure 4.5. (Note that our convention in this paper is that twist boxes represent vertical twists).

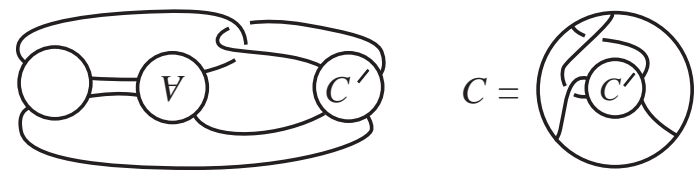

Figure 4.4

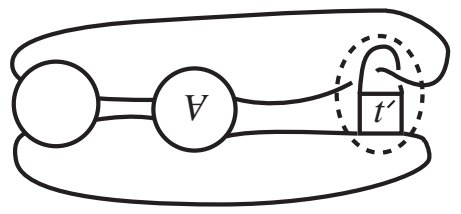

Figure 4.5

The tangle encapsulated in the Conway sphere in Figure 4.5 is $\mathcal{R}\left(2, t^{\prime}, 0\right)=\mathcal{R}\left(\frac{2}{2 t^{\prime}+1}\right)$. Thus either $t^{\prime}=0,-1$ or $A=\mathcal{R}(s)$ for some $s \in \mathbb{Z}$. In either case, $C=\mathcal{R}\left(t^{\prime}, 1,1,0\right)=$ $\mathcal{R}(t, 2,0)$ where $t=-\left(t^{\prime}+1\right)$.

Figure 4.6 shows $\mathcal{C}\left(A, *, C, \frac{1}{2}\right)$, where $C=\mathcal{R}(t, 2,0)$ corresponds to the rational number $\frac{t}{2 t+1}$. Thus $\mathcal{C}\left(A, *, C, \frac{1}{2}\right)$ orbifold-toroidal implies that $\Delta\left(\frac{1}{0}, \frac{t}{2 t+1}\right)>1$. Thus $|2 t+1|>1$. Consequently, $t^{\prime}=-(t+1) \neq-1,0$ and $A=\mathcal{R}(s)$. Again, that $\mathcal{C}\left(A, *, C, \frac{1}{2}\right)$ is orbifold-toroidal guarantees that $|s|>1$. 


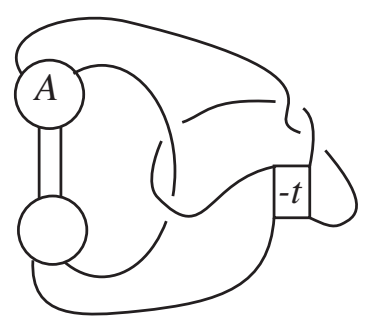

Figure 4.6

Lemma 4.5 If $\mathcal{C}\left(A, B, *, \frac{1}{0}\right)$ is a rational tangle then, up to symmetry exchanging $A$ and $B, A=\mathcal{R}(s)$ and $B=\mathcal{R}(t,-s)$ for $s, t \in \mathbb{Z}$. If $\mathcal{C}\left(A, B, *, \frac{1}{2}\right)$ is orbifold-toroidal then $|s|>1, t \neq 0$ and $(s, t) \neq(2,1),(-2,-1)$.

Proof Isotoping $\mathcal{C}\left(A, B, *, \frac{1}{0}\right)$ to Figure 4.7 we see that one of $A$ or $B$ must be an integral tangle. By symmetry we assume it is $A, A=\mathcal{R}(s)$. Thus we are as in Figure 4.8, from which we see that $B^{\prime}=\mathcal{R}(1 / t)$. Thus $B=\mathcal{R}(t,-s)$. Now assume $\mathcal{C}\left(A, B, *, \frac{1}{2}\right)$ is orbifold-toroidal. See Figure 4.9. Then $|s|>1$, and $B=\mathcal{R}(t,-s)$ corresponds to tangle slope $\frac{1-s t}{t}$. Thus $\Delta\left(\frac{0}{1}, \frac{1-s t}{t}\right)>1$. That is, $|1-s t|>1$. Thus $t \neq 0$ and $(s, t) \neq(2,1),(-2,-1)$.

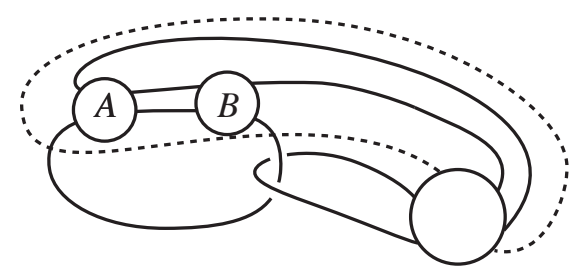

Figure 4.7
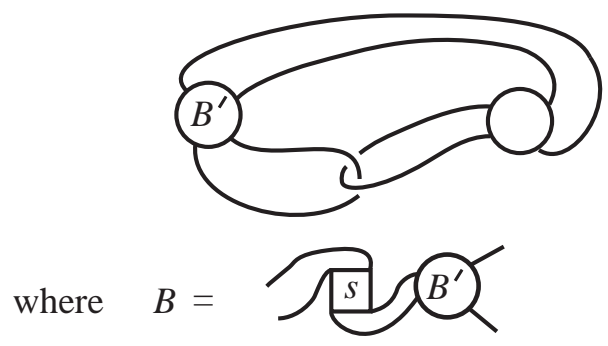

Figure 4.8 


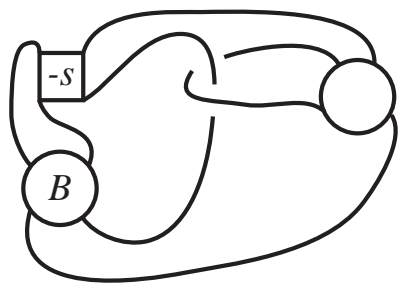

Figure 4.9

Proof of Theorem 4.1 We prove this for $J_{1}(\ell, m)$. The proof for $J_{2}(\ell, m)$ is similar. Recall that by attaching the appropriate " $C$ "-tangle to $\mathcal{T}_{1}(\ell, m)$ we get the tangle $B(\ell, m, n, 0)$ of [6]. There are infinitely many such fillings corresponding to different values of $n$. Looking at double branched covers, this says that the corresponding Dehn fillings of $X$, the exterior of $J_{1}(\ell, m)$, are the exteriors of the hyperbolic knots $k(\ell, m, n, 0)$. Denote the two components of $\partial X$ as $\partial_{1} X, \partial_{2} X$, where $\partial_{1} X$ is the component along which these fillings are made (corresponding to the boundary of the ambient solid torus of $J_{1}(\ell, m)$ ). Because infinitely many fillings of $X$ are hyperbolic, either $X$ is hyperbolic or there is a cable space along $\partial_{1} X$ (Theorem 2.4.4 of [5]). We assume the latter for contradiction. Then the slope of each of these Dehn fillings is distance 1 from a unique slope $\gamma$, and furthermore, Dehn filling $X$ along $\gamma$ has a lens space summand. The $\mathbb{Z}_{2}$-orbifold quotient of the $\gamma$-Dehn filling of $X$ is pictured in Figure 4.10 (the $C$-tangle for this picture corresponds to replacing the $n$ twist box of $B(\ell, m, n, 0)$ with $\mathcal{R}(1 / 0))$. But inserting $\mathcal{R}(1 / 0)$ into the " $D$ "-tangle of Figure 4.10 gives the unlink of two components. That is, there is a filling of $\partial_{2} X$ such that, along with the filling of $\partial_{1} X$ along $\gamma$, gives $S^{2} \times S^{1}$. But this contradicts the fact that first filling $X$ along $\gamma$ yields a lens space summand.

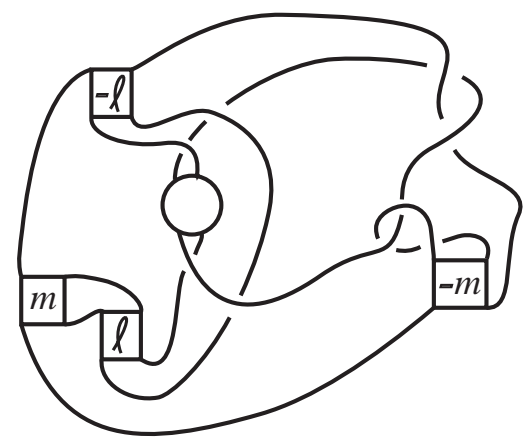

Figure 4.10 


\section{Non-integral surgery and the JSJ-decomposition}

In this section we consider a non-integral Dehn filling $X(\gamma)$ on the exterior $X$ of a knot in $S^{3}$, and analyze the relation between the JSJ-decompositions of $X$ and $X(\gamma)$.

If $M$ is an irreducible 3-manifold we shall denote by $\operatorname{Seif}(M)$ the disjoint union of the Seifert fibered pieces of the JSJ-decomposition of $M$. In the case of a knot exterior $X$, the possible components of $\operatorname{Seif}(X)$ have been described by Jaco and Shalen.

Lemma 5.1 [14, Lemma VI.3.4] Let $X$ be the exterior of a knot in $S^{3}$, and let $W$ be a component of $\operatorname{Seif}(X)$. Then $W$ is either a torus knot space, a cable space, or a composing space.

The relation between $\mathbf{T}(X)$ and $\mathbf{T}(X(\gamma))$ is described in the following theorem and its addendum.

Theorem 5.2 Let $X$ be the exterior of a knot $k$ in $S^{3}$, and suppose $\Delta(\gamma, \mu) \geq 2$ where $\mu$ is the meridian of $k$. Let $W$ be the component of $X$ cut along $\mathbf{T}(X)$ that contains $\partial X$. Then exactly one of the following four possibilities holds.

(1) $\mathbf{T}(X(\gamma))=\mathbf{T}(X)$;

(2) $\mathbf{T}(X)=\varnothing, X$ is hyperbolic, $k$ is an Eudave-Muñoz knot $k(\ell, m, n, p)$, $\Delta(\gamma, \mu)=2, X(\gamma)=M_{1} \cup_{T} M_{2}$, where $M_{i}$ is a Seifert fiber space over $D^{2}$ with two exceptional fibers, $i=1,2$, and $\mathbf{T}(X(\gamma))=T$;

(3) $k$ is contained in a tubular neighborhood $N\left(k_{0}\right)$ of a non-trivial knot $k_{0}$ as a $J_{\varepsilon}(\ell, m)$-satellite of $k_{0}, \partial W=T_{0} \cup \partial X$, where $T_{0}=\partial N\left(k_{0}\right), \Delta(\gamma, \mu)=2$, $W(\gamma)=N_{1} \cup_{T} M_{2}$, where $N_{1}$ is a Seifert fiber space over $A^{2}$ with one exceptional fiber and $M_{2}$ is a Seifert fiber space over $D^{2}$ with two exceptional fibers, and $\mathbf{T}(X(\gamma))=\mathbf{T}(X) \cup T$;

(4) $k$ is a $(p, q)$-cable of a non-trivial knot $k_{0}$ with exterior $X_{0}, q \geq 2, \partial W=$ $T_{0} \cup \partial X$ as in (3), $\gamma=\frac{n p q \pm 1}{n}, n \geq 2$, and $\mathbf{T}(X(\gamma))=\mathbf{T}(X)-T_{0}$.

We spell out more details about cases (1) and (4) of Theorem 5.2 in the following addendum, where $k_{\gamma}$ denotes the core of the Dehn filling solid torus in $X(\gamma)$.

Addendum 5.3 In case (1) of Theorem 5.2 we have

(a) if $W=X$ then $X(\gamma)$ is atoroidal;

(b) if $W \neq X$ then $W(\gamma)$ is hyperbolic if and only if $W$ is hyperbolic; 
(c) if $W \neq X$ then $W(\gamma)$ is Seifert fibered if and only if $W$ is Seifert fibered. If $W$ is a cable space then $W(\gamma)$ is a Seifert fiber space over $D^{2}$ with two exceptional fibers, and if $W$ is a composing space with $(n+1)$ boundary components then $W(\gamma)$ is a Seifert fiber space over an $n$-punctured sphere with a single exceptional fiber of multiplicity $\Delta(\gamma, \mu)$. In both cases $k_{\gamma}$ is isotopic to an exceptional fiber of $W(\gamma)$.

In case (4) of Theorem 5.2 we have

(a) $W$ is a cable space and $W(\gamma)$ is a solid torus, with meridian $\gamma_{0}$, say, on $T_{0}$;

(b) conclusion (1) holds for $X_{0}\left(\gamma_{0}\right)$;

(c) $k_{\gamma}$ is isotopic to an $(r, s)$-cable of the core of $W(\gamma)$, for some $s>1$.

\section{Proof of Theorem 5.2}

Case I $|\partial W|=1$

Here $\mathbf{T}(X)=\varnothing$ and $W=X$, so $X$ is either hyperbolic or Seifert fibered. In the first case, either (1) or (2) holds by [11]. In the second case, $k$ is a torus knot, $X(\gamma)$ is a Seifert fiber space over $S^{2}$ with at most three exceptional fibers, and $\mathbf{T}(X(\gamma))=\mathbf{T}(X)=\varnothing$.

Case II $|\partial W|=2$

There are two subcases.

\section{(A) $W$ hyperbolic}

$W(\mu)$ is a solid torus; therefore $W(\gamma)$ is irreducible and $\partial$-irreducible, by [29] and [5] respectively. If $W(\gamma)$ is hyperbolic then $\mathbf{T}(X(\gamma))=\mathbf{T}(X)$, and (1) holds. By [23, Proposition 9], $W(\gamma)$ is not Seifert fibered. Hence we may assume that $W(\gamma)$ is toroidal. Then by Theorem $4.2 \mathrm{k}$ is a $J_{\varepsilon}(\ell, m)$-satellite in $W(\mu) \cong S^{1} \times D^{2}$. Then $W(\gamma)=N_{1} \cup_{T} M_{2}$ as in conclusion (3). Let $\varphi$ be the fiber of $N_{1}$ on $T_{0}=\partial W(\gamma)$.

Consider the component $Z \neq W$ of the JSJ-decomposition of $X$ with $T_{0} \subset \partial Z$. Assume that $Z$ is a Seifert fiber space, with fiber $\psi$ on $T_{0}$. We will show that $\psi \neq \varphi$; hence $\mathbf{T}(X(\gamma))=\mathbf{T}(X) \cup T$ and (3) holds.

Let $\mu_{0}$ be the meridian of $W(\mu) \cong S^{1} \times D^{2}$. Since $\Delta\left(\mu_{0}, \varphi\right) \geq 2$ by the Addendum to Theorem 4.3, it suffices to prove that $\Delta\left(\mu_{0}, \psi\right) \leq 1$.

By Lemma 5.1, $Z$ is either (i) a torus knot exterior, or (ii) a cable space, or (iii) a composing space. Let $X_{0}=\overline{X-W}$; so $\partial X_{0}=T_{0}$. Since $X_{0}\left(\mu_{0}\right) \cong S^{3}$, we must 
have $\Delta\left(\mu_{0}, \psi\right)=1$ in case (i), $\Delta\left(\mu_{0}, \psi\right)=1$ in case (ii) (since $Z \cup W(\mu)$ is a solid torus), and, $\mu_{0}=\psi$ in case (iii) (since $\partial(Z \cup W(\mu))$ must be compressible).

\section{(B) $W$ Seifert fibered.}

Then $W$ is a cable space. Thus $k$ is a $(p, q)$-cable, $q \geq 2$, of a non-trivial knot $k_{0}$. Let $\varphi$ be the slope on $\partial X$ of the Seifert fiber of $W$; thus $\varphi=p q / 1$ with respect to the usual meridian-longitude basis.

If $\Delta(\gamma, \varphi) \geq 2$ then $W(\gamma)$ is a Seifert fiber space over $D^{2}$ with two exceptional fibers, which is atoroidal. Therefore $\mathbf{T}(X(\gamma))=\mathbf{T}(X)$.

Since $\Delta(\gamma, \mu) \geq 2(\mu=1 / 0=$ meridian of $k), \gamma \neq \varphi$. So assume $\Delta(\gamma, \varphi)=1$; ie, $\gamma=\frac{n p q \pm 1}{n}, n \geq 2$. Then $W(\gamma)$ is a solid torus, with meridian $\gamma_{0}$, say, on $T_{0}$. Let $\mu_{0}$ be the slope on $T_{0}$ of the meridian of the solid torus $W(\mu)$. Then $\Delta\left(\gamma_{0}, \mu_{0}\right)=q^{2} \Delta(\gamma, \mu) \geq 8$. Let $X_{0}=\overline{X-W}$, the exterior of $k_{0}$. By induction on the number of components of $\mathbf{T}(X)$, we may assume that the theorem holds for $X_{0}$. (The start of the induction is Case (1) above.) Since $\Delta\left(\gamma_{0}, \mu_{0}\right)>2$, conclusions (2) and (3) of the theorem do not hold for $X_{0}$. If (1) holds for $X_{0}$, then we get $\mathbf{T}(X(\gamma))=$ $\mathbf{T}\left(X_{0}\left(\gamma_{0}\right)\right)=\mathbf{T}\left(X_{0}\right)=\mathbf{T}(X)-T_{0}$, which is conclusion (4) for $X$. Finally, assume that (4) holds for $X_{0}$. So $k_{0}$ is a $\left(p_{1}, q_{1}\right)$-cable, $q_{1} \geq 2$, of a non-trivial knot $k_{1}$, and $\gamma_{0}=\frac{n_{1} p_{1} q_{1} \pm 1}{n_{1}}, n_{1} \geq 2$, with respect to the usual basis for $k_{0}$. But we also have $\gamma_{0}=\frac{n p q \pm 1}{n q^{2}}, n \geq 2$, and this is a contradiction (see [8, page 704]).

Case III $|\partial W| \geq 3$

Let the components of $\partial W-\partial X$ be $T_{1}, \ldots, T_{n}, n \geq 2$. Then the components of $\overline{X-W}$ are $Y_{1}, \ldots, Y_{n}$, say, where $\partial Y_{i}=T_{i}$ and $T_{i}$ is incompressible in $Y_{i}, 1 \leq i \leq n$. Since $T_{i}$ compresses in $S^{3}=X(\mu)$, it compresses in $W(\mu)$. Hence $W(\mu)$ is reducible. (In fact it is easy to show that $W(\mu)$ is a connected sum of $n$ solid tori.)

Again we distinguish two subcases.

\section{(A) $W$ hyperbolic}

Since $\Delta(\gamma, \mu) \geq 2, W(\gamma)$ is irreducible by [10]. Also, since $|\partial W| \geq 3, W(\gamma)$ is atoroidal and anannular by [37]. Therefore $W(\gamma)$ is hyperbolic, and $\mathbf{T}(X(\gamma))=\mathbf{T}(X)$. This is conclusion (1).

\section{(B) $W$ Seifert fibered}

Since $|\partial W|=n+1, n \geq 2, W$ is a composing space by Lemma 5.1. Also, the meridian $\mu$ is the Seifert fiber of $W$, since $W(\mu)$ is reducible. Hence $W(\gamma)$ is a Seifert fiber space over an $n$-punctured sphere with one exceptional fiber, of multiplicity $\Delta(\gamma, \mu)$. Since the Seifert fibers of $W$ and $W(\gamma)$ are the same on each $T_{i}$, we have $\mathbf{T}(X(\gamma))=\mathbf{T}(X)$, and (1) holds. 
Proof of Addendum 5.3 This follows by examining the proof of Theorem 5.2.

\section{Main theorem}

Recall from Section 2 the definition of the characteristic 2-sided toric 2-suborbifold $\mathbf{T}(K)$ of a prime knot $K$ in $S^{3}$. Let $\operatorname{Seif}(K)=\operatorname{Seif}(O(K))$ be the disjoint union of the $S^{1}$-fibered components of $O(K)$ cut along $\mathbf{T}(K)$.

Let $(a, \partial a)$ be an unknotting arc for $K$. As described in Section 2, a relative regular neighborhood of $(a, \partial a)$ in $\left(S^{3}, K\right)$ determines a marked tangle $\mathcal{T}_{0}$ which is replaced with a tangle $\mathcal{T}_{0}^{\prime}$ under the crossing move. Let $M$ be the double branched cover of $S^{3}$ along $K, V_{0}$ be the solid torus preimage of $\mathcal{T}_{0}$ under the branched covering, and $X=M-V_{0}$. Then $M=X(\gamma)$ and $S^{3}=X(\mu)$ where $\Delta(\mu, \gamma)=2$. Let $k=k_{\mu}$ be the knot in $S^{3}$ of which $X$ is the exterior, and let $k_{\gamma}$ be the core of $V_{0}$ in $M$.

Definition The unknotting arc $(a, \partial a)$ is said to be an $(r, s)$-cable of an exceptional fiber of $\operatorname{Seif}(K)$ iff $k_{\gamma}$ is an $(r, s)$-cable of an exceptional fiber in $\operatorname{Seif}(M)$.

Remark If $(a, \partial a)$ is an $(r, s)$-cable of an exceptional fiber of $\operatorname{Seif}(K)$, then the corresponding $\mathcal{T}_{0}$ lies in a rational tangle $\mathcal{R}(p / q)$ in $\operatorname{Seif}(K)$ which is the quotient of a neighborhood of this exceptional fiber. The tangle $\mathcal{R}(p / q)-\mathcal{T}_{0}$ in $S^{2} \times I$, has a double branched cover which is a cable space. By Lemma 3.8, $\mathcal{R}(p / q)-\mathcal{T}_{0}$ is homeomorphic to $\mathcal{M}\left(\frac{v}{w}, *\right)$ for some $v, w \in \mathbb{Z}$. The results of applying the crossing move associated to such an $(a, \partial a)$ are further discussed in Lemmas 8.1, 12.3, 12.4, and Theorem 8.2.

Lemma 6.1 Let $(a, \partial a)$ be an unknotting arc for $K$. One of the following holds:

(1) $(a, \partial a)$ can be isotoped in $\left(S^{3}, K\right)$ to be disjoint from $\mathbf{T}(K)$. Furthermore, if $\mathbf{T}(K) \neq \varnothing$ and $(a, \partial a)$ can be isotoped into $\operatorname{Seif}(K)$, then $a$ is isotopic to an $(r, s)$-cable of an exceptional fiber in $\operatorname{Seif}(K)$, for some $s \geq 1$.

(2) (a) $K$ is an EM-knot $K(\ell, m, n, p)$.

(b) $O(K)$ has a unique connected, incompressible, 2-sided, toric 2-suborbifold $S$, a Conway sphere, $K$ has an unknotting arc $(b, \partial b)$ with $|b \cap S|=1$ (the standard unknotting arc for $K(\ell, m, n, p))$, and no unknotting arc is disjoint from $S$.

(3) $K$ is the union of essential tangles $\mathcal{P} \cup \mathcal{P}_{0}$, where $\mathcal{P}_{0}$ is the EM-tangle $\mathcal{A}_{\varepsilon}(\ell, m)$, $\partial \mathcal{P}_{0} \subset \mathbf{T}(K)$, and $(a, \partial a)$ can be isotoped into $\mathcal{P}_{0}$. If $\mathcal{T}(*, *)$ is the exterior in $\mathcal{P}_{0}$ of the crossing ball corresponding to $(a, \partial a)$, then $\mathcal{T}(*, *)$ is as described in Theorem 4.3. 
Proof of Lemma 6.1 Let $M, k=k_{\mu}, k_{\gamma}$ be as described above. We are now in the context of Theorem 5.2. Possibilities (1), (2), and (3) in the conclusion of Theorem 5.2 will lead to conclusions (1), (2), and (3), respectively of Lemma 6.1, and possibility (4) will lead to conclusion (1).

Let $h: M \rightarrow M$ be the covering involution, with quotient orbifold $\widehat{M}=O(K)$. Write $V_{0}=V_{\gamma}$, with quotient $\widehat{V}_{\gamma}$ the 3-suborbifold $\mathcal{T}_{0}$ of $O(K)$.

(1) Here $\mathbf{T}(M)=\mathbf{T}(X)$. The covering involution $h: M \rightarrow M$ restricts to $h: X \rightarrow X$, and we can isotop $\mathbf{T}(X)$ in $X$ to be $h$-invariant [20]. Let $T$ be a component of $\mathbf{T}(X)$. Then $T$ separates $X$ into two components, one of which contains $\partial X$. It follows that if $h(T)=T$ then $h$ preserves the sides of $T$, and hence $\mathbf{T}^{+}(M)=\mathbf{T}^{+}(X)=\mathbf{T}(X)=$ $\mathbf{T}(M)$.

By (1)(a) of Addendum 5.3, if $\mathbf{T}(X)=\varnothing$ then $M=X(\gamma)$ is atoroidal, and hence $\mathbf{T}(K)=\varnothing$. Thus conclusion (1) holds trivially.

If $\mathbf{T}(X) \neq \varnothing$, then $X=X_{0} \cup W$, say, $X_{0} \neq \varnothing$. Hence $M=X_{0} \cup W(\gamma)$, and, taking quotients, $O(K)=\widehat{X}_{0} \cup \widehat{W}(\gamma)$, where $\widehat{V}_{\gamma}=\mathcal{T}_{0} \subset \widehat{W}(\gamma)$. By (1)(b) of Addendum 5.3, if $W$ is hyperbolic then $W(\gamma)$ is hyperbolic, and so $\widehat{W}(\gamma)$ is atoroidal and is a component of $O(K)$ cut along $\mathbf{T}(K)$. Hence $\mathbf{T}(K)$ can be orbifold-isotoped off $\widehat{W}(\gamma)$, in particular, off $\mathcal{T}_{0}$. If $W$ is Seifert fibered, then by (1)(c) of Addendum 5.3 the Seifert fibering of $W$ extends to a Seifert fibering of $W(\gamma)$. Thus $\widehat{W}(\gamma)$ is $S^{1}-$ fibered, and $\widehat{V}_{\gamma}=\mathcal{T}_{0}$ is a neighborhood of an exceptional (orbifold) fiber. Also, since $\mathbf{T}(M)=\mathbf{T}(X), W(\gamma)$ is a component of $\operatorname{Seif}(M)$, and hence $\widehat{W}(\gamma)$ is a component of $\operatorname{Seif}(K)$.

(2) Here $k^{*}$ is an Eudave-Muñoz knot $k(\ell, m, n, p)$. By Theorem 3.4(1), $K=$ $K(\ell, m, n, p), M=M_{1} \cup_{T} M_{2}, \mathbf{T}(M)=T$, and we may isotop $T$ so that $h\left(M_{i}\right)=$ $M_{i}, i=1,2$. Hence $\mathbf{T}(K)=S=\widehat{T}$. The facts that $K$ has an unknotting $\operatorname{arc} b$ with $|b \cap S|=1$, and no unknotting arc disjoint from $S$, are proved in [6].

(3) Here $k^{*}$ is a $J_{\varepsilon}(\ell, m)$-satellite of $k_{0}$. Thus $X=X_{0} \cup_{T_{0}} W$, where $X_{0}$ is the exterior of $k_{0}, T_{0}=\partial X_{0}$, and $W$ is the exterior of $J_{\varepsilon}(\ell, m)$ in $S^{1} \times D^{2}$. Also, $M=X_{0} \cup_{T_{0}} W(\gamma), W(\gamma) \cong N_{1} \cup_{T} M_{2}$ as in Theorem 5.2, and $\mathbf{T}(M)=\mathbf{T}(X) \cup T$. Since $W$ is hyperbolic, $T_{0} \subset \mathbf{T}(X)$.

Now $h: M \rightarrow M$ leaves $X$ invariant. Hence we can isotop $\mathbf{T}(X)$ in $X$ to be $h-$ invariant [20]. In particular, we must clearly have $h\left(T_{0}\right)=T_{0}$. Hence $h$ leaves $W(\gamma)$ invariant. Fix $(h)$ cannot be disjoint from $T_{0}$ or completely lie in $T_{0}$, otherwise $h$ would give rise to an involution on $S^{3}$ whose fixed set was $k_{0}$ or a satellite of $k_{0}$-contradicting the $\mathbb{Z}_{2}-$ Smith Conjecture [36]. In particular the quotient of $W$ 
under $h$, the exterior of the crossing ball corresponding to $(a, \partial a)$, is a tangle $\mathcal{T}(*, *)$ in $S^{2} \times I$ satisfying the hypotheses of Theorem 4.3; hence $\mathcal{T}(*, *)$ is as described there. This implies $\mathcal{P}_{0} \cong \widehat{W}(\gamma)=\mathcal{T}(*, \beta) \cong \mathcal{A}_{\varepsilon}(\ell, m)$ for some $\varepsilon, \ell, m$. Since $\mathbf{T}(M)=\mathbf{T}(X) \cup T, N_{1}$ and $M_{2}$ of $W(\gamma)$ are components of Seif $(M)$. Furthermore, $\mathbf{T}^{+}(M)=\mathbf{T}(M)$ since $h$ preserves the sides of $T$ and since $\mathbf{T}^{+}(X)=\mathbf{T}(X)$ by the argument for (1). Thus $\partial \mathcal{P}_{0} \in \mathbf{T}(K)$ and $\widehat{N}_{1}, \widehat{M}_{2}$ are components of $\operatorname{Seif}(K)$.

(4) Here $W$ is a cable space and $W(\gamma)$ is a solid torus. Let $X_{0}=\overline{X-W}$, and $\partial X_{0}=T_{0}$. Then $M=X_{0} \cup_{T_{0}} W(\gamma)$, and $\mathbf{T}(M)=\mathbf{T}(X)-T_{0}=\mathbf{T}\left(X_{0}\right)$. Since $h$ leaves $X$ invariant, we can isotop $\mathbf{T}(X)$ in $X$ to be $h$-invariant. Then $h\left(T_{0}\right)=T_{0}$, $\mathbf{T}\left(X_{0}\right)$ is $h$-invariant, and $\mathbf{T}^{+}(M)=\mathbf{T}^{+}\left(X_{0}\right)=\mathbf{T}\left(X_{0}\right)=\mathbf{T}(M)$. Therefore $\mathbf{T}(K)$ is the quotient $\widehat{\mathbf{T}}\left(X_{0}\right)$.

Let $\mu_{0}$ be the meridian of $K_{0}$ on $T_{0}$, and let $\gamma_{0}$ be the meridian of the solid torus $W(\gamma)$. Then $\Delta\left(\gamma_{0}, \mu_{0}\right)>2$, and $M=X(\gamma)=X_{0}\left(\gamma_{0}\right)$. Let $W_{0}$ be the component of $X_{0}$ cut along $\mathbf{T}\left(X_{0}\right)$ that contains $\partial X_{0}$. By case (4)(b) of Addendum 5.3, (1) holds for $X_{0}\left(\gamma_{0}\right)$; thus (1)(a), (1)(b) and (1)(c) of Addendum 5.3 hold for $X_{0}, W_{0}$. Conclusions $1(\mathrm{a})$ and 1 (b) now follow from the argument in case (1) above applied to $X_{0}, W_{0}, \gamma_{0}$.

Theorem 6.2 Let $K$ be a knot with unknotting number 1. Then one of the following three possibilities holds.

(1) (a) Any unknotting arc $(a, \partial a)$ for $K$ can be isotoped in $\left(S^{3}, K\right)$ so that $a \cap \mathbf{T}(K)=\varnothing$.

(b) If $\mathbf{T}(K) \neq \varnothing$ and $K$ has an unknotting arc $a$ in $\operatorname{Seif}(K)$ then $a$ is isotopic to an $(r, s)$-cable of an exceptional fiber of $\operatorname{Seif}(K)$, for some $s \geq 1$.

(2) (a) $K$ is an EM-knot $K(\ell, m, n, p)$.

(b) $O(K)$ has a unique connected incompressible 2-sided toric 2-suborbifold $S$, a Conway sphere, $K$ has an unknotting arc $a$ with $|a \cap S|=1$ (the standard unknotting arc for $K(\ell, m, n, p))$, and $K$ has no unknotting arc disjoint from $S$.

(3) $K$ is the union of essential tangles $\mathcal{P} \cup \mathcal{P}_{0}$, where $\mathcal{P}_{0}$ is an EM-tangle $\mathcal{A}_{\varepsilon}(\ell, m)$ and $\partial \mathcal{P}_{0}$ is in $\mathbf{T}(K)$. Any unknotting arc for $K$ can be isotoped into $\mathcal{P}_{0}$. The standard unknotting arc for $\mathcal{A}_{\varepsilon}(\ell, m)$ is an unknotting arc for $K$.

Remarks (A) If $K=K(\ell, m, n, p)$, then (2) must hold. If $K=\mathcal{P} \cup \mathcal{P}_{0}$ where $\mathcal{P}_{0}$ is an EM-tangle then (1) or (3) may hold. If (1) holds then, still, by Lemma 9.6 (and Definition 3.3), any unknotting arc of $K$ can be isotoped into $\mathcal{P}_{0}$ (hence into an exceptional fiber of $\mathcal{P}_{0}$ ). 
(B) In conclusion (3), to say that $K$ is unknotted by the standard unknotting arc for $\mathcal{A}_{\varepsilon}(\ell, m)$ (as described in Section 4) we mean that there is a tangle homeomorphism from $\mathcal{P}_{0}$ to $\mathcal{A}_{\varepsilon}(\ell, m)$ which makes this identification. Any two such will differ by an isotopy of the tangle ball fixed on the boundary, which will isotop the two unknotting arcs. Indeed, any homeomorphism of tangles, $h: \mathcal{A}_{\varepsilon}(\ell, m) \rightarrow \mathcal{A}_{\varepsilon}(\ell, m)$, preserves the markings, hence is isotopic to the identity.

Question Is any unknotting arc for $K(\ell, m, n, p)$ or for $\mathcal{A}_{\varepsilon}(\ell, m)$ isotopic to its standard unknotting arc?

One approach to the above question would be to prove an analog of Theorem 3.4 for the exteriors of $k(\ell, m, n, p), J_{\varepsilon}(\ell, m)$ (resp.). That is, show that there is a unique tangle quotient arising from involutions on any such knot exterior.

Proof of Theorem 6.2 Theorem 6.2(2) is the same as Lemma 6.1(2). So we assume $K$ is not an EM-knot. Furthermore we may assume that $K$ can be written as the union of essential tangles $\mathcal{P} \cup \mathcal{P}_{0}$ with $\mathcal{P}_{0}$ homeomorphic to $\mathcal{A}_{\varepsilon}(\ell, m)$ and $\partial \mathcal{P}_{0} \subset \mathbf{T}(K)$ (otherwise (1) holds for $K$ by Lemma 6.1).

If $(a, \partial a)$ is an unknotting arc for $K$ which cannot be isotoped into $\mathcal{P}_{0}$, then Lemma 6.1(1) applies. But this says the unknot can be written as $\mathcal{T} \cup \mathcal{A}_{\varepsilon}(\ell, m)$ for some tangle $\mathcal{T}$ and some $\varepsilon, \ell, m$. This contradicts Lemma 9.6 and Definition 3.3. Thus any unknotting arc for $K$ can be isotoped into $\mathcal{P}_{0}$.

If conclusion (1) of Theorem 6.2 does not hold then there is an unknotting arc $(a, \partial a)$ satisfying Lemma 6.1(3). Let $\mathcal{T}(*, *)$ be the exterior of the crossing ball corresponding to $(a, \partial a)$ and $h_{1}: \mathcal{T}(*, \beta) \rightarrow \mathcal{T}_{\varepsilon}(\ell, m)\left(\frac{1}{2}\right), h_{2}: \mathcal{T}(*, \alpha) \rightarrow \mathcal{T}_{\varepsilon}(\ell, m)\left(\frac{1}{0}\right)$ be the tangle homeomorphisms provided by Theorem 4.3. The standard unknotting arc for $\mathcal{A}_{\varepsilon}(\ell, m)$ corresponds to the crossing move $\mathcal{T}_{\varepsilon}(\ell, m)\left(\frac{1}{2}\right) \rightarrow \mathcal{T}_{\varepsilon}(\ell, m)\left(\frac{1}{0}\right)$. Thus $h_{1}$ identifies the standard unknotting arc of $\mathcal{P}_{0} \cong \mathcal{A}_{\varepsilon}(\ell, m)$ for which we are looking. That is, performing the crossing move on $K$ dictated by the standard unknotting arc gives the knot gotten by gluing $\mathcal{T}_{\varepsilon}(\ell, m)\left(\frac{1}{0}\right)$ to $\mathcal{P}$ via $h_{1}^{-1} \mid \partial$. Since $\left(h_{2} \mid \partial\right)\left(h_{1} \mid \partial\right)^{-1}$ is the identity, this is the same as gluing $\mathcal{T}_{\varepsilon}(\ell, m)\left(\frac{1}{0}\right)$ to $\mathcal{P}$ via $h_{2}^{-1} \mid \partial$, which is the unknot by assumption.

The following is a generalization of the result of [30; 31] (see also [17]) that an unknotting arc for a satellite knot can always be taken to be disjoint from the companion 2-torus.

Corollary 6.3 Let $K$ be a knot with unknotting number 1, that is neither an EMknot nor a knot containing an EM-tangle with essential boundary. Let $F$ be an 
incompressible 2-sided toric 2-suborbifold of $O(K)$. Then any unknotting arc $(a, \partial a)$ for $K$ can be isotoped in $\left(S^{3}, K\right)$ so that $a \cap F=\varnothing$.

This is an immediate consequence of Theorem 6.2 and the following lemma.

Lemma 6.4 Let $F$ be an incompressible 2-sided toric 2-suborbifold of $O(K)$. Then $F$ is orbifold isotopic to a vertical suborbifold of $\operatorname{Seif}(K)$.

Proof The fact that $F$ is isotopic into $\operatorname{Seif}(K)$ follows from the discussion in [3] beginning at the paragraph immediately preceding Lemma 7 on page 456, and ending at the statement "and therefore that $F \cap F^{\prime}=\varnothing$ " near the bottom of page 457.

So assume $F \subset \operatorname{Seif}(K)$. Any component of $F$ that is boundary parallel in $\operatorname{Seif}(K)$ can be isotoped to be vertical, so by [3, Verticalization Theorem 4] it is enough to show that $F$ cannot be isotoped to be horizontal. Let $p: M \rightarrow O(K)$ be the double branched covering projection. If $F$ were a horizontal 2-suborbifold of $O(K)$, then $p^{-1}(F)$ would be a horizontal surface in $M$. But $H_{2}(M ; \mathbb{Q})=0$, so $M$ contains no horizontal surface.

\section{Mutation}

Let $\mathcal{T}=(B, A)$ be a knot in $S^{3}$ or a tangle. Let $\mathcal{T}_{0}=\left(B_{0}, A_{0}\right)$ be a subtangle of $\mathcal{T}$ such that $B_{0}$ is a 3-ball. Let $S_{0}=\partial B_{0}$. Let $h: B_{0} \rightarrow B^{3}$ be a homeomorphism such that $h\left(S_{0} \cap A\right)=Q \subset S^{2}$. Let $\Gamma_{0} \cong \mathbb{Z}_{2} \times \mathbb{Z}_{2}$ be the group of automorphisms of $\left(S^{2}, Q\right)$ consisting of rotations through $\pi$ about any one of the three co-ordinate axes in $\mathbb{R}^{3}$ together with the identity. Let $\mathcal{T}_{1}=\mathcal{T}-\operatorname{int} \mathcal{T}_{0}$ and regard $\mathcal{T}$ as $\mathcal{T}_{0} \cup \mathcal{T}_{1}$, where $\mathcal{T}_{0}$ is glued to $\mathcal{T}_{1}$ by the identity map on $S_{0}$. Now for $g \in \Gamma_{0}-\{1\}$, let $\mu=h^{-1} g h: S_{0} \rightarrow S_{0}$ and define $\mathcal{T}^{\prime}=\mathcal{T}_{0} \cup_{\mu} \mathcal{T}_{1}$. We say $\mathcal{T}^{\prime}$ is a mutant of $\mathcal{T}$. The operation of replacing $\mathcal{T}$ by $\mathcal{T}^{\prime}$ is mutation of $\mathcal{T}$ along $S_{0}$, by the mutation involution $\mu$.

Boileau has asked [16, Problem 1.69(c)] if the unknotting number of a link is a mutation invariant. We prove that this is at least true for knots with unknotting number 1.

Theorem 7.1 Having unknotting number 1 is invariant under mutation.

Proof Let $K$ be a knot with unknotting number 1 , and let $K^{\prime}$ be a mutant of $K$. Then there is a Conway sphere $S$ which decomposes $K$ into two tangles $\mathcal{T}_{1}$ and $\mathcal{T}_{2}$, such that $K^{\prime}=\mathcal{T}_{1} \cup \rho\left(\mathcal{T}_{2}\right)$, where $\rho$ is rotation of the ball $B_{2}$ containing $\mathcal{T}_{2}$ through $\pi$ about one of the co-ordinate axes. Note that we also have $K^{\prime}=\rho\left(\mathcal{T}_{1}\right) \cup \mathcal{T}_{2}$. If either 
$\mathcal{T}_{1}$ or $\mathcal{T}_{2}$ is trivial then $K=K^{\prime}$. Also, since $u(K)=1, K$ is prime [28]. Hence we may assume that the tangles $\mathcal{T}_{i}$ are prime, and therefore that $S$ is essential.

First suppose that $K$ is not an EM-knot nor the union of two essential tangles, one of which is an EM-tangle. Then, taking $F=S$ in Corollary 6.3 we get that there is an unknotting arc $a$ for $K$ disjoint from $S$, and therefore, without loss of generality, contained in $B_{2}$. As marked tangles, the crossing move determined by $a$ transforms $\mathcal{T}_{2}$ to a rational tangle $\mathcal{R}$. Then the crossing move on $K^{\prime}$ determined by $\rho(a)$ transforms $\rho\left(\mathcal{T}_{2}\right)$ to the rational tangle $\rho(\mathcal{R})=\mathcal{R}$, and hence transforms $K^{\prime}$ to $\mathcal{T}_{1} \cup \mathcal{R}=$ unknot. If $K$ is an EM-knot, then $K^{\prime}=K[6]$.

Finally, suppose $K$ is the union of essential tangles $\mathcal{P} \cup \mathcal{P}_{0}$, where $\mathcal{P}_{0}$ is an EM-tangle. Then $\mathcal{P}_{0}$ is of the form $\mathcal{S}(\alpha, \beta ; \gamma, *)$. If $S$ is not isotopic in $O(K)$ to $S_{0}=\partial \mathcal{M}(\alpha, \beta)$, then the argument above shows that $K^{\prime}$ has unknotting number 1 . So assume that $S=S_{0}$. Since rotating $\mathcal{M}(\alpha, \beta)$ about the horizontal axis leaves it invariant, we may assume that $\rho$ is rotation about the axis perpendicular to the plane of the paper. Thus $K^{\prime}=\mathcal{P} \cup \mathcal{P}_{0}^{\prime}$, where $\mathcal{P}_{0}^{\prime}=\mathcal{S}(\beta, \alpha ; \gamma, *)$.

By part (3) of Theorem 6.2, $K$ has an unknotting arc $a$ that lies in the 3-ball $B_{0}$ containing the tangle $\mathcal{P}_{0}$. By Lemma 2.1, there is a homeomorphism $h: \mathcal{S}(\alpha, \beta ; \gamma, *) \rightarrow$ $\mathcal{S}(\beta, \alpha ; \gamma, *)$ such that $h \mid \partial D$ is rotation through $\pi$ about the horizontal axis. Note that $h$ is isotopic to the corresponding rotation of the ball $\overline{S^{3}-D}$. Hence there is a rotation $g$ of the ball $B_{0}$ which takes $\mathcal{P}_{0}$ to $\mathcal{P}_{0}^{\prime}$. The crossing change of $K$ determined by $a$ converts $\mathcal{P}_{0}$ to a rational tangle $\mathcal{R}$, where $\mathcal{P} \cup \mathcal{R}$ is the unknot. Therefore the crossing change of $K^{\prime}$ determined by $g(a)$ converts $K^{\prime}=\mathcal{P} \cup \mathcal{P}_{0}^{\prime}=\mathcal{P} \cup g\left(\mathcal{P}_{0}\right)$ to $\mathcal{P} \cup g(\mathcal{R})=\mathcal{P} \cup \mathcal{R}=$ unknot.

\section{Algebraic knots}

For the definition of an algebraic knot or link (in the sense of Conway) see Section 2 of [33]. We briefly summarize this in a form suitable for our present purposes.

An elementary (algebraic) tangle is a tangle of the form $\mathcal{M}(\alpha, \beta), \mathcal{M}(\gamma, *)$, or $\mathcal{M}(*, *)$, where $\alpha, \beta, \gamma \in \mathbb{Q}-\mathbb{Z}$. We shall refer to these as elementary tangles of type I, II, or III respectively. See Figure 8.1.

A Montesinos tangle of length $3, \mathcal{M}(\alpha, \beta, \gamma), \alpha, \beta, \gamma \in \mathbb{Q}-\mathbb{Z}$, is defined in the obvious way; see Figure 8.2.

Recall [4] that if $\mathcal{T}$ is a marked tangle in $B^{3}$ then $1^{*} \mathcal{T}$ is the knot or link obtained by capping off $\mathcal{T}$ with the rational tangle $\mathcal{R}(0)$ (ie, $1^{*} \mathcal{T}$ is the numerator closure of $\mathcal{T}$ ).

Then an algebraic knot is a knot of one of the following forms: 


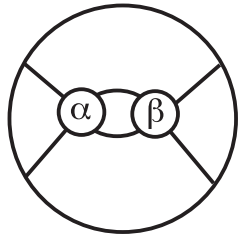

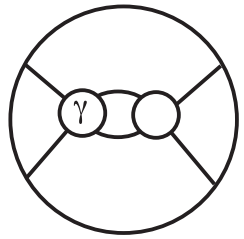

II

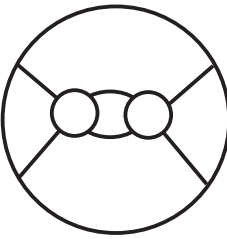

III

Figure 8.1

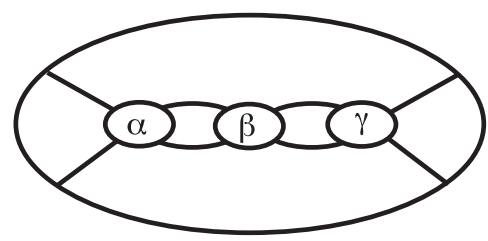

Figure 8.2

(a) $1^{*} \mathcal{R}(\alpha)$;

(b) $1^{*} \mathcal{M}(\alpha, \beta, \gamma)$;

(c) a union along boundary components of elementary tangles.

The knots of type (a) are the 2-bridge or rational knots. (Any knot $1^{*} \mathcal{M}(\alpha, \beta)$ can also be expressed as $1^{*} \mathcal{R}(\gamma)$.) Those of type (b) are the Montesinos knots of length 3. We will call a knot of type (c) a large algebraic knot. Thus an algebraic knot is large if and only if it has an essential Conway sphere. If $K$ is an algebraic knot, of type (a), (b), or (c), then the double branched cover of $K$ is a lens space, a Seifert fiber space over $S^{2}$ with three exceptional fibers, or a toroidal graph-manifold, respectively.

An elementary tangle comes equipped with a marking, given by Figure 8.1. In constructing a large algebraic knot $K$, the gluing homeomorphisms between the boundary components of the elementary tangles will not in general preserve the markings. To describe $K$ as a marking-preserving union of marked tangles we need to interpolate marked tangles of 4-string braids in $S^{2} \times I$ between the boundary components.

This can also be described in terms of diagrams. Figure 8.1 III is a diagram in a pair of pants of an elementary tangle of type III. A diagram of an elementary tangle of type I or II, in a disk or annulus respectively, may be obtained by inserting diagrams of the appropriate rational tangles into the diagrams in Figure 8.1, I or II. Also, a 4-string braid in $S^{2} \times I$ has a diagram in an annulus. Then a knot is large algebraic if and only if it has a diagram in $S^{3}$ that is a union along boundary components of such elementary tangle diagrams and 4-string braid diagrams. 
Lemma 8.1 $\mathcal{R}(p / q)$ can be transformed to $\mathcal{R}(1 / 0)$ by a crossing move if and only if there exist coprime integers $r, s$ such that $p / q=\frac{2 r s \pm 1}{2 s^{2}}$.

Proof If $p / q=\frac{2 r s \pm 1}{2 s^{2}}, q \neq 0$, then $p / q$ has a continued fraction expansion of the form $\left[a_{1}, a_{2}, \ldots, a_{k}, \pm 2,-a_{k}, \ldots,,-a_{2},-a_{1}, a\right]$, (see [15] or [18]), and hence can be transformed to $\mathcal{R}(1 / 0)$ by a crossing move.

Conversely, suppose $\mathcal{R}(p / q)$ can be transformed to $\mathcal{R}(1 / 0)$ by a crossing move. Let the double branched covers of $\mathcal{R}(1 / 0)$ and $\mathcal{R}(p / q)$ be $V$ and $V^{\prime}$ respectively. Then there is a knot $K$ in $V$ such that (with respect to some framing of $K$ ), $m / 2-$ Dehn surgery on $K$ gives $V^{\prime}$. Note that, with respect to the basis of $H_{1}(\partial V)$ corresponding to the standard marking, the meridian of $V^{\prime}$ has slope $p / q$.

If $K$ is unknotted in $V$, then $p / q=1 / 0$, while if $K$ is a core of $V$, then $p / q=m / 2$; in both cases $p / q$ is of the stated form.

Otherwise, it follows from [5, Theorem 2.4.4] that $K$ is an $(r, s)$-cable of the core of $V$. With respect to the usual framing on $K$, the Seifert fiber of the cable space $V$ - int $N(K)$ has slope $r s$ on $\partial N(K)$. Hence, $K(m / 2)$ will be a solid torus $V^{\prime}$ if and only if $\Delta(m / 2, r s / 1)=1$, ie $m=2 r s \pm 1$. The meridian of $V^{\prime}$ then has slope $m / 2 s^{2}=\frac{2 r s \pm 1}{2 s^{2}}$

Theorem 8.2 Let $K$ be a large algebraic knot with unknotting number 1. Then either

(1) any unknotting arc for $K$ can be isotoped into either

(a) one of the rational tangles $\mathcal{R}(p / q)$ in an elementary tangle of type I; or

(b) the rational tangle $\mathcal{R}(p / q)$ in an elementary tangle of type II.

In case (a), the crossing move transforms $\mathcal{R}(p / q)$ to $\mathcal{R}(k / 1)$ for some integer $k$, and $p / q=\frac{2 s^{2}}{2 r s \pm 1}+k$, where $s \geq 1$ and $(r, s)=1$.

In case (b), the crossing move transforms $\mathcal{R}(p / q)$ to $\mathcal{R}(1 / 0)$, and $p / q=\frac{2 r s \pm 1}{2 s^{2}}$, where $s \geq 1$ and $(r, s)=1$.

(2) (a) $K$ is an $E M-k n o t ~ K(\ell, m, n, p)$.

(b) $O(K)$ has a unique connected incompressible 2-sided toric 2-suborbifold $S$, a Conway sphere, $K$ has an unknotting arc $a$ with $|a \cap S|=1$ (the standard unknotting arc for $K(\ell, m, n, p))$, and $K$ has no unknotting arc disjoint from $S$.

(3) $K$ is the union of essential tangles $\mathcal{P} \cup \mathcal{P}_{0}$, where $\mathcal{P}_{0}$ is an EM-tangle $\mathcal{A}_{\varepsilon}(\ell, m)$ and $\partial \mathcal{P}_{0}$ is in $\mathbf{T}(K)$. Any unknotting arc for $K$ can be isotoped into $\mathcal{P}_{0}$. The standard unknotting arc for $\mathcal{A}_{\varepsilon}(\ell, m)$ is an unknotting arc for $K$. 
Remark The remarks (A), (B) following Theorem 6.2 also apply here.

Proof Note that the characteristic orbifold decomposition of $O(K)$ is gotten by amalgamating subcollections of the constituent elementary tangles. Applying Theorem 6.2, we are left to check that Theorem 6.2(1) implies Theorem 8.2(1). Theorem 6.2(1) implies that the unknotting move replaces $\mathcal{R}(p / q)$ in some elementary tangle of type I or II with another tangle $\mathcal{T}$. $\mathcal{T}$ must be an integer tangle, $\mathcal{R}(k / 1)$, if the elementary tangle is of type I, and $\mathcal{R}(1 / 0)$ if it is of type II. Lemma 8.1 gives the desired result (using $\mathcal{R}\left(1 /\left(\frac{p}{q}-k\right)\right)$ for $\mathcal{R}(p / q)$ in type I).

\section{Some algebraic tangle calculations}

In this section we do some calculations concerning crossing moves on certain algebraic tangles. These will be used in Sections 10 and 11.

Lemma 9.1 Suppose $q>1$, and that $\mathcal{M}(p / q, \chi)$ is a rational tangle, where $\chi \in$ $\mathbb{Q} \cup\{\infty\}$. Then

(1) $\chi=k \in \mathbb{Z}$;

(2) $\mathcal{M}(p / q, k)=\mathcal{R}\left(\frac{k q+p}{q}\right)$

(3) if $\mathcal{M}(p / q, k)=\mathcal{R}(1 / x), x \in \mathbb{Z}$, then there exists $\varepsilon= \pm 1$ such that $x=\varepsilon q$ and $k q+p=\varepsilon$.

Proof (1) Since $\mathcal{M}(p / q, \chi)$ is a disk sum of $\mathcal{R}(p / q)$ and $\mathcal{R}(\chi)$, and $q>1$, we must have $\chi=k \in \mathbb{Z}$.

(2) Incorporating the $k$ horizontal twists into $\mathcal{R}(p / q)$, we see that $\mathcal{M}(p / q, \chi)=$ $\mathcal{R}\left(\frac{k q+p}{q}\right)$.

(3) This follows immediately from (2).

Lemma 9.2 Suppose $q_{1}, q_{2}>1$, and that $\mathcal{M}\left(p_{1} / q_{1}, p_{2} / q_{2}\right)$ can be transformed to a rational tangle $\mathcal{R}$ by a crossing move. Then

(1) the crossing arc is isotopic to an $(r, s)$-cable, $s \geq 1$, of one of the two exceptional fibers of $\mathcal{M}\left(p_{1} / q_{1}, p_{2} / q_{2}\right)$;

(2) the crossing move transforms the corresponding rational tangle, $\mathcal{R}\left(\frac{p_{1}}{q_{1}}\right)$, say, of $\mathcal{M}$, to an integral tangle $\mathcal{R}(k)$;

(3) $\mathcal{R}=\mathcal{R}\left(\frac{k q_{2}+p_{2}}{q_{2}}\right)$; 
(4) $p_{1} / q_{1}=k+\frac{2 s^{2}}{2 r s \pm 1}$.

Proof (1) The argument is very similar to the proofs of Lemma 6.1 and Theorem 5.2. Let $M$ be the double branched cover of $B^{3}$ along $\mathcal{M}\left(\frac{p_{1}}{q_{1}}, \frac{p_{2}}{q_{2}}\right) . M$ is a Seifert fiber space over the disk with two exceptional fibers. The crossing move corresponds to replacing a marked tangle $\mathcal{T}_{0}$ in $\mathcal{M}\left(\frac{p_{1}}{q_{1}}, \frac{p_{2}}{q_{2}}\right)$ with a marked tangle $\mathcal{T}_{0}^{\prime}$, resulting in a rational tangle. Let $V_{0}$ be the solid torus preimage of $\mathcal{T}_{0}$ in $M$ and $X=M-\operatorname{int}\left(V_{0}\right)$ be its exterior. Let $\gamma$ be the meridian of $V_{0}$. Then $M=X(\gamma)$ and $X(\mu)$ is a solid torus for some $\mu$ with $\Delta(\gamma, \mu)=2$. By [23, Proposition 9], $X$ must be either Seifert fibered or toroidal. In the first case, $X$ is the exterior of an exceptional fiber in $M$ and we are done. So $X$ is toroidal and we let $W$ be the component of $X$ cut along $\mathbf{T}(X)$ (canonical torus decomposition) that contains $\partial X$. Then $\partial W-\partial X$ is compressible in $W(\gamma), W(\mu)$. Furthermore $W(\gamma), W(\mu)$ are irreducible. Then $W(\gamma), W(\mu)$ are solid tori. By [5, Theorem 2.4.4], $W$ is a cable space. In particular, say that $W$ is the exterior of the $(p, q)$ curve in the solid torus $W(\mu), q \geq 2$. If $\gamma_{0}, \mu_{0}$ are the slopes of the meridian disks on $\partial W(\gamma), \partial W(\mu)$ respectively, then $\Delta\left(\gamma_{0}, \mu\right)=q^{2}(\Delta \gamma, \mu) \geq 8$. Thus, if $X_{0}=X-W$, we may argue as above to conclude that $X_{0}$ is the exterior of a $(p, q)$-cable on some knot $k_{0}$ in $X(\mu)$. But then a coordinate calculation, as in the proof of Theorem 5.2 (Case IIB), says that $\gamma_{0}=\frac{n_{1} p_{1} q_{1} \pm 1}{n_{1}}, n_{1} \geq 2$ and $\gamma_{0}=\frac{n p q \pm 1}{n q^{2}}$ for $n \geq 2$. This contradiction finishes the proof of Lemma 9.2(1).

Lemma 9.2(2) and (3) now follow from Lemma 9.1, (1) and (2). Finally, (4) follows from Lemma 8.1 applied to $\mathcal{R}\left(1 /\left(\frac{p_{1}}{q_{1}}-k\right)\right)$.

Corollary 9.3 Suppose $q_{1}, q_{2}>1$ and that $\mathcal{M}\left(p_{1} / q_{1}, p_{2} / q_{2}\right)$ can be transformed to a vertical twist tangle $\mathcal{R}(1 / x)$ by a crossing move. Then there exist $\varepsilon= \pm 1$ and $k \in \mathbb{Z}$ such that, after possibly interchanging $p_{1} / q_{1}$ and $p_{2} / q_{2}$,

(1) $x=\varepsilon q_{2}$;

(2) $k q_{2}+p_{2}=\varepsilon$

(3) $p_{1} / q_{1}=k+\frac{2 s^{2}}{2 r s \pm 1}$, for some $s \geq 1,(r, s)=1$.

Proof This follows from Lemmas 9.1 and 9.2.

Lemma 9.4 $1^{*} \mathcal{M}(\alpha, \beta)$ is the unknot if and only if $\Delta(\alpha,-\beta)=1$.

Proof $1^{*} \mathcal{M}(\alpha, \beta)$ is the unknot if and only if its double branched cover $M$ is $S^{3}$. But $M$ is the union of the two solid tori $\tilde{\mathcal{R}}(\alpha)$ and $\widetilde{\mathcal{R}}(\beta)$, whose meridians have slopes $\alpha$ and $-\beta$ respectively on the torus $T=\partial \widetilde{\mathcal{R}}(\alpha)$ with respect to the basis of $H_{1}(T)$ corresponding to the lifts of the slopes $1 / 0$ and $0 / 1$ on $\partial \mathcal{R}(\alpha)$. Hence $M$ is $S^{3}$ if and only if $\Delta(\alpha,-\beta)=1$. 
Lemma 9.5 Let $K$ be the knot shown in Figure 9.1, where $q_{1}, q_{2}>1$ and $\mathcal{T}$ is some marked tangle. Then

(1) $K$ is the unknot if and only if $\mathcal{T}=\mathcal{R}(x)$, where $x \in \mathbb{Z}$ satisfies

$$
x q_{1} q_{2}+p_{1} q_{2}+p_{2} q_{1}= \pm 1
$$

(2) if $\left|p_{1} / q_{1}\right|,\left|p_{2} / q_{2}\right|<1$, and $x$ is as in (1), then $|x| \leq 1$.

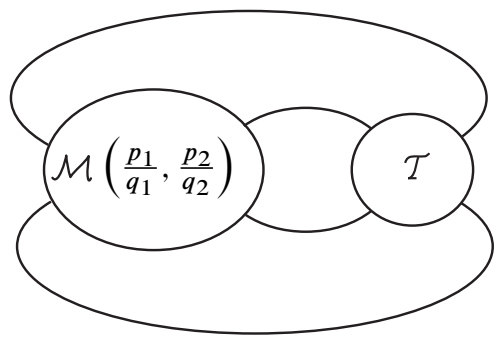

Figure 9.1

Proof Suppose $K$ is the unknot. Passing to double branched covers, we see that $\tilde{\mathcal{T}}$ must be a solid torus, implying that $\mathcal{T}$ is a rational tangle. Moreover, the meridian of $\widetilde{\mathcal{T}}$ must be distance 1 from the Seifert fiber of $\tilde{\mathcal{M}}\left(p_{1} / q_{1}, p_{2} / q_{2}\right)$. Since the latter projects to the slope $1 / 0$ on $\partial \mathcal{M}\left(p_{1} / q_{1}, p_{2} / q_{2}\right)$, this implies that $\mathcal{T}=\mathcal{R}(x)$ for some $x \in \mathbb{Z}$. Incorporating this twist tangle $\mathcal{R}(x)$ with $\mathcal{R}\left(p_{1} / q_{1}\right)$, we see that the unknot is the union of the rational tangles $\mathcal{R}\left(x+\frac{p_{1}}{q_{1}}\right)=\mathcal{R}\left(\frac{x q_{1}+p_{1}}{q_{1}}\right)$ and $\mathcal{R}\left(p_{2} / q_{2}\right)$. Then $\Delta\left(\frac{x q_{1}+p_{1}}{q_{1}}, \frac{-p_{2}}{q_{2}}\right)=1$ by Lemma 9.4, giving the equation in (1).

Conversely, if $\mathcal{T}=\mathcal{R}(x)$ where $x$ satisfies the given equation then $\Delta\left(\frac{x q_{1}+p_{1}}{q_{1}},-\frac{p_{2}}{q_{2}}\right)=$ 1 and $K$ is the unknot by Lemma 9.4.

To prove (2), suppose $\left|p_{1} / q_{1}\right|,\left|p_{2} / q_{2}\right|<1$. Then from (1) we have

$$
x+\frac{p_{1}}{q_{1}}+\frac{p_{2}}{q_{2}}= \pm \frac{1}{q_{1} q_{2}},
$$

giving $|x| \leq \frac{\left(q_{1}-1\right)}{q_{1}}+\frac{\left(q_{2}-1\right)}{q_{2}}+\frac{1}{q_{1} q_{2}}<2$.

Lemma 9.6 Suppose $\alpha, \beta, \gamma \in \mathbb{Q}-\mathbb{Z}$, and $|\alpha|,|\beta|<1$. Then $\mathcal{S}(\alpha, \beta ; \gamma, \mathcal{T})$ is not the unknot, for any tangle $\mathcal{T}$. 
Proof Suppose $\mathcal{S}(\alpha, \beta ; \gamma, \mathcal{T})$ is the unknot. Then passing to double branched coverings as usual we see that $\mathcal{T}$ must be a rational tangle $\mathcal{R}(\chi)$. Furthermore, by Lemma 9.5, $\mathcal{M}(\gamma, \chi)$ must be a vertical twist tangle $\mathcal{R}(-1 / x)$, where $|x| \leq 1$ since $|\alpha|,|\beta|<1$. But by Lemma 9.1(3), $|x| \geq 2$.

Corollary 9.7 Let $K=\mathcal{S}(\alpha, \beta ; \gamma, \delta)$, where $\alpha, \beta, \gamma, \delta \in \mathbb{Q}-\mathbb{Z}$, and $|\alpha|,|\beta|<1$. Then $K$ cannot be unknotted by a crossing move in $\mathcal{M}(\gamma, \delta)$.

Proof By Lemma 9.2(2), the crossing move has the effect of replacing one of the rational tangles $\mathcal{R}(\gamma)$ or $\mathcal{R}(\delta)$ in $\mathcal{M}(\gamma, \delta)$ with some other (rational) tangle. But this contradicts Lemmas 9.6 and 2.2(2).

Recall (Corollary 3.2) that the EM-knots are all of the form $\mathcal{S}(\alpha, \beta ; \gamma, \delta)$ with $\alpha, \beta, \gamma, \delta$ $\in \mathbb{Q}-\mathbb{Z}$ and $|\alpha|,|\beta|,|\gamma|,|\delta|<1$.

Theorem 9.8 Let $K=\mathcal{S}(\alpha, \beta ; \gamma, \delta)$ where $\alpha, \beta, \gamma, \delta \in \mathbb{Q}-\mathbb{Z}$ and $|\alpha|,|\beta|,|\gamma|,|\delta|<1$. Then $K$ has unknotting number 1 if and only if $K$ is an EM-knot $K(\ell, m, n, p)$.

Proof $K$ has a unique essential Conway sphere $S=\partial \mathcal{M}(\alpha, \beta)=\partial \mathcal{M}(\gamma, \delta)$. Therefore, by Corollary 6.3, if $K$ has unknotting number 1 then either $K$ is an EM-knot or $K$ can be unknotted by a crossing move disjoint from $S$. But the latter is impossible by Corollary 9.7 .

\section{Examples}

In this section we apply our results to certain families of knots defined in terms of the notation of Conway [4]; since that notation naturally encodes the characteristic toric orbifold decomposition of a knot it is eminently suited to our techniques. In particular, we consider all the knots up to 11 crossings in Conway's tables [4] with the property that their description in the tables makes it clear that they contain an essential Conway sphere. It turns our that these are all large algebraic knots. Specifically, they are the knots that are listed in [4] as either .a.b, .a.b.c, .a.b.c.d, .a. $(b, c), .(a, b) . c$, $(a, b)(c, d),(a, b) 1(c, d)$, or $a, b, c, d$. We determine exactly which of them have unknotting number 1 . Note that the knots $a, b, c, d$ are Montesinos knots of length 4 , so they have unknotting number greater than 1 by [25].

Throughout this section, $a, b, c$ and $d$ will denote rational numbers.

We start with the knots .a.b.c.d. Recall [4, page 335] that in Conway's tables the form a.b.c.d is used only when $a, b, c$ and $d$ are $>0$. It turns out that all the EM-knots 
$K(\ell, m, n, p)$ are of this form (up to mirror image), and that a knot .a.b.c.d with $a, b, c, d>0$ has unknotting number 1 if and only if it is an EM-knot. Recall also that in [4] a.b.c. 1 is abbreviated to .a.b.c, and a.b.1 to .a.b.

First we describe some symmetries of Conway's .x.y.z.w notation. Recall that if $x, y, z$ and $w$ are arbitrary marked tangles, then $. x . y . z . w$ is the knot shown in Figure 10.1 (where the leftmost and rightmost horizontal arcs are understood to meet at the point at infinity in the projection $S^{2}$ ).

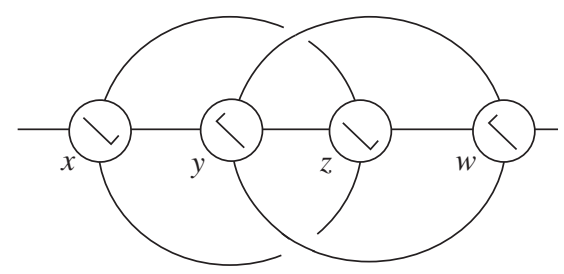

Figure 10.1

Let $D_{8}$ be the dihedral group of order 8 , the group of symmetries of the square. By cyclically numbering the vertices of the square $1,2,3,4$, we regard $D_{8}$ as a subgroup of $S_{4}$. Then $D_{8}$ acts on the set of expressions of the form .x.y.z.w by permuting the substituent tangles; thus, by a slight abuse of notation, we write $\pi\left(. x_{1} \cdot x_{2} \cdot x_{3} \cdot x_{4}\right)=$ $\cdot x_{\pi(1)} \cdot x_{\pi(2)} \cdot x_{\pi(3)} \cdot x_{\pi(4)}$, for $\pi \in D_{8}$.

Recall also [4, pages 330-331] that $\mathbb{Z}_{2} \times \mathbb{Z}_{2}$ acts on the set of marked tangles as follows. If $x$ is a marked tangle, then $x_{h}, x_{v}$ and $x_{r}$ are the marked tangles obtained by rotating $x$ through $180^{\circ}$ about the horizontal axis, the vertical axis, and the axis perpendicular to the plane of the paper, respectively. Let Mut $\cong \mathbb{Z}_{2} \times \mathbb{Z}_{2}$ be the group $\{h, v, r$, id $\}$. (Then mutation is the equivalence relation on knots generated by replacing a tangle $x$ in (some diagram of) $K$ by $x_{f}$ for some $f \in$ Mut.) Again by a slight abuse of notation, we write $(. x \cdot y \cdot z \cdot w)_{f}=. x_{f} \cdot y_{f} \cdot z_{f} \cdot w_{f}$, for $f \in$ Mut.

Let $\mu: D_{8} \rightarrow$ Mut be the epimorphism defined by $\mu\left(\left(\begin{array}{lll}1 & 2 & 3\end{array}\right)\right)=h$, and $\mu((14)(23))=$ $v$. Finally, let $\varepsilon:$ Mut $\rightarrow \mathbb{Z}_{2}=\{ \pm 1\}$ be the homomorphism defined by $\varepsilon(h)=\varepsilon(v)=-1$, and recall that - denotes mirror-image.

Theorem 10.1 If $\pi \in D_{8}$ then:

$$
\pi(. x . y . z . w)=\varepsilon(\mu(\pi))(. x \cdot y \cdot z \cdot w)_{\mu(\pi)}
$$

Corollary 10.2 Up to mutation and mirror-image, .x.y.z.w is invariant under the action of $D_{8}$. 
Since $t_{f}=t$ for rational tangles $t$, for all $f \in$ Mut, we have

Corollary 10.3 If $\pi \in D_{8}$ then:

$$
\pi(. a . b . c . d)=\varepsilon(\mu(\pi)) . a . b . c . d
$$

Proof of Theorem 10.1 Consider .x.y.z.w, as shown in Figure 10.1. By sliding the tangle $x$ around the point at infinity we get Figure 10.2. Changing all crossings, we see that $. x \cdot y \cdot z \cdot w=-\cdot y_{h} \cdot z_{h} \cdot w_{h} \cdot x_{h}$. This shows that the theorem holds for $\pi=\left(\begin{array}{lll}1 & 2 & 3\end{array}\right)$.

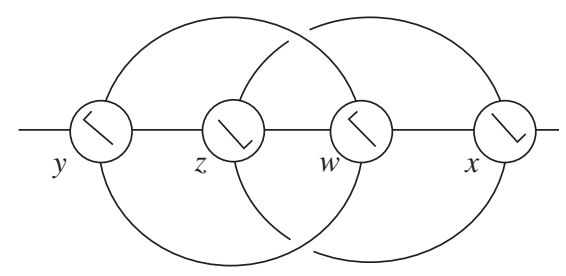

Figure 10.2

Rotating Figure 10.1 through $180^{\circ}$ about the central axis perpendicular to the plane of the paper, we get Figure 10.3. Changing all crossings shows that $x . y . z . w=$ .$- w_{v} \cdot z_{v} \cdot y_{v} \cdot x_{v}$, in other words, the theorem holds for $\pi=(14)(23)$.

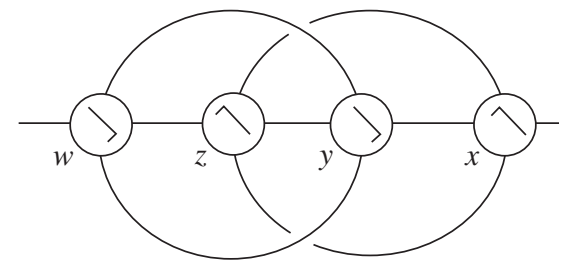

Figure 10.3

Since (1 234 ) and (14)(23) generate $D_{8}$, the result follows.

We consider a further symmetry. Recall [4, page 331] that $t 0$ denotes the reflection of the tangle $t$ in a plane perpendicular to the paper through the NW/SE-diagonal. Note that $(t 0)_{r}$ is then the reflection of $t$ in a plane through the NE/SW-diagonal.

Theorem $10.4 \quad . x . y . z . w=-. x 0 .(y 0)_{r} \cdot z 0 .(w 0)_{r}$

In particular, for rational tangles we have

Corollary 10.5 a $a 0 . b 0 . c 0 . d 0=-$.a.b.c.d 
Proof $t 00=t$ and $a_{r}=a$ when $a$ is rational.

\section{Corollary 10.6}

(1) $. a . b . c 0=-(. a 0 . b 0 . c)$

(2) $. a . b=-(. a 0 . b 0)$

(3) $. a 0 . b=-(. a . b 0)$

Proof These follow immediately from Corollary 10.5, along with the facts that $10=1$ and $t 00=t$.

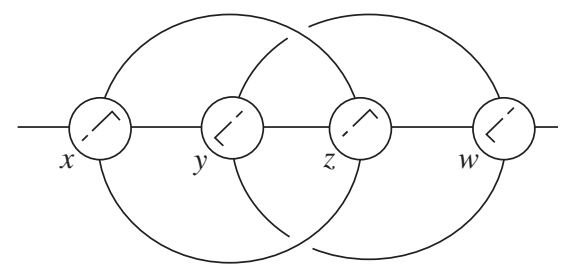

Figure 10.4

Proof of Theorem 10.4 Rotating Figure 10.1 through $180^{\circ}$ about the horizontal axis gives Figure 10.4. Changing all crossings, we now get .x0.(y0) $r . z 0 .(w 0)_{r}$.

The following lemma describes a.b.c.d in terms of the square tangle $\mathcal{S}$.

Lemma 10.7 a.b.c.d $=\mathcal{S}\left(\frac{-1}{c+1}, \frac{a}{a+1} ; \frac{1}{b+1}, \frac{-d}{d+1}\right)$

Proof This follows from the second deformation shown in [4, Figure 10], possibly together with Lemma 2.2.

Recall that if $K$ is an EM-knot $K(\ell, m, n, p)$, then, by taking the mirror image of $K$ if necessary, we may assume that $\ell>1$.

Lemma 10.8 Assume $\ell>1$. Then $K(\ell, m, n, p)=. a . b . c$, where $a, b, c$ are $>0$ and are given by:

$$
\begin{aligned}
& p=0: \quad a=\frac{m}{(\ell-1) m-1}, \quad b=\frac{2 m n-m+n}{2 m n-m-n+1}, \quad c=\ell-1 \\
& n=0: \quad a=\frac{2 m p-m-p}{(\ell-1)(2 m p-m-p)-2 p+1}, \quad b=\frac{m}{m-1}, \quad c=\ell-1
\end{aligned}
$$


Proof This follows from Lemmas 3.1 and 10.7.

Theorem 10.9 Let $K=$.a.b.c.d, with $a, b, c, d>0$. Then $K$ has unknotting number 1 if and only if it is an EM-knot $K(\ell, m, n, p)$.

Proof By Lemma 10.7, a.b.c.d $=\mathcal{S}(\alpha, \beta ; \gamma, \delta)$ where $\alpha, \beta, \gamma, \delta \in \mathbb{Q}-\mathbb{Z}$ and $|\alpha|,|\beta|,|\gamma|,|\delta|<1$. The result is now an immediate consequence of Theorem 9.8.

We now determine the EM-knots up to 11 crossings.

Theorem 10.10 The EM-knots $K(\ell, m, n, p)$ with at most 11 crossings are listed in Table EM 11, up to mirror image.

\begin{tabular}{c|l|r} 
Rolfsen & Conway & $K(\ell, m, n, p)$ \\
\hline $8_{17}$ & .2 .2 & $K(3,2,0,1)$ \\
$9_{33}$ & .21 .2 & $-K(2,3,0,1)$ \\
$10_{82}$ & .4 .2 & $K(2,2,0,2)$ \\
$10_{84}$ & .22 .2 & $K(2,2,0,-1)$ \\
$10_{88}$ & .21 .21 & $K(2,3,0,0)$ \\
$10_{91}$ & .3 .2 .20 & $-K(4,1,1,0)$ \\
$10_{95}$ & .210 .2 .2 & $K(3,2,0,0)$ \\
& .311 .2 & $-K(2,2,-1,0)$ \\
& .23 .2 & $K(2,2,0,3)$ \\
& .212 .2 & $K(2,2,0,-2)$ \\
& .2111 .2 & $-K(2,2,2,0)$ \\
& .31 .21 & $-K(2,-3,1,0)$ \\
& .22 .2 .20 & $-K(3,-2,1,0)$ \\
& .210 .21 .2 & $K(3,2,1,0)$ \\
\hline
\end{tabular}

$K(\ell, m, n, p)$ with at most 11 crossings

Table EM 11

Remark The third column of Table EM 11 represents the knot in $K(\ell, m, n, p)$ form. These representations are not unique.

Proof of Theorem 10.10 By possibly taking mirror images, Proposition 1.4 of [6] allows us to assume that $\ell>1$. By Lemma $10.8, K(\ell, m, n, p)=. a . b . c$ with $a, b, c$ positive rational numbers. Inserting alternating diagrams of the corresponding rational tangles $\mathcal{R}(a), \mathcal{R}(b), \mathcal{R}(c)$ into Conway's $6^{* *}$ polyhedron gives an alternating diagram of a.b.c. This alternating diagram will be a minimal crossing diagram from which we 
can compute the crossing number of .a.b.c. To get alternating diagrams of the rational tangles we can use the positive continued fraction expansions of $a, b$, and $c$. That is, if $r$ is a positive rational number and $r=\left[a_{1}, a_{2}, \ldots, a_{n}\right]$ (see Section 2) with $a_{i}>0$ if $i \neq n$ and $a_{n} \geq 0$, then there is an alternating diagram of the rational tangle $\mathcal{R}(r)$ that has exactly $\sum_{i=1}^{n} a_{i}$ crossings. If $r \neq 1$, we will take $a_{1}>1$.

In Tables E1-E3 below, we list nonnegative continued fraction expansions of $a, b, c$.

\begin{tabular}{c|c|c|c}
$m, p$ & $a$ & $b$ & $c$ \\
\hline$m>0, p>0$ & {$[p-1,2, m-2,1, \ell-2,0]$} & {$[m-1,1]$} & $\ell-1$ \\
$m>0, p<0$ & {$[|p|, 1,1, m-2,1, \ell-2,0]$} & {$[m-1,1]$} & $\ell-1$ \\
$m<0, p>0$ & {$[p-1,1,1,|m|, \ell-1,0]$} & {$[|m|, 1,0]$} & $\ell-1$ \\
$m<0, p<0$ & {$[|p|, 2,|m|, \ell-1,0]$} & {$[|m|, 1,0]$} & $\ell-1$ \\
\multicolumn{2}{c|}{$n=0$} & {$[\ell>1, \quad m \neq 0,1, \quad(\ell, m, p) \neq(2,2,1)]$} &
\end{tabular}

Table E1

\begin{tabular}{c|c|c|c}
$m, n$ & $a$ & $b$ & $c$ \\
\hline$m>0, n>0$ & {$[m-1,1, \ell-2,0]$} & {$[n-1,1,1, m-1,1]$} & $\ell-1$ \\
$m>0, n<0$ & {$[m-1,1, \ell-2,0]$} & {$[|n|, 2, m-1,1]$} & $\ell-1$ \\
$m<0, n>0$ & {$[|m|, \ell-1,0]$} & {$[n-1,2,|m|-1,1,0]$} & $\ell-1$ \\
$m<0, n<0$ & {$[|m|, \ell-1,0]$} & {$[|n|, 1,1,|m|-1,1,0]$} & $\ell-1$ \\
\multicolumn{2}{|l|}{$\quad[\ell>1}$, & $m \neq 0, \quad(\ell, m) \neq(2,1) ;(m, n) \neq(1,0),(-1,1)]$
\end{tabular}

Table E2

\begin{tabular}{c|c|c|c}
$m$ & $a$ & $b$ & $c$ \\
\hline$m>0$ & {$[m-1,1, \ell-2,0]$} & {$[m-1,1]$} & $\ell-1$ \\
$m<0$ & {$[|m|, \ell-1,0]$} & {$[|m|, 1,0]$} & $\ell-1$
\end{tabular}

$n=0=p \quad(\ell>1 ; m \neq 0,1)$

Table E3

If 0 appears in any but the last entry of one of these expansions (for certain $\ell, m, n, p$ ), we may use one of the following rules to eliminate it:

(1) $[0, a, b, c, \cdots] \longrightarrow[b, c, \cdots]$

(2) $[\cdots a, b, 0, c, d, \cdots] \longrightarrow[\cdots a, b+c, d, \cdots]$

Note that the sum of the entries is changed by (1) only and that amounts to deleting the second entry from the sum. In this way we enumerate those $K(\ell, m, n, p)$ with crossing number at most 11 .

Algebraic $6 \mathcal{G}$ Geometric $\mathcal{T}$ opology, Volume 6 (2006) 
As an example, consider the case when $n=0, m>0, p>0$. Assuming $p>1$, Table E1 gives the crossing number of $K(\ell, m, 0, p)$ as $2 m+2 \ell+p$ (the crossings from $\mathcal{R}(a)$, $\mathcal{R}(b), \mathcal{R}(c)$ plus 3 from the Conway polyhedron $\left.6^{* *}\right)$. Thus if $K(\ell, m, n, p)$ has at most 11 crossings, $(\ell, m, p) \in\{(2,2,2),(2,2,3)\}$ (noting that $m \neq 1$ when $n=0$ ). If $p=1$ and $m>2$, then the crossing number of $K(\ell, m, 0,1)$ is $2 m+2 \ell-1$. Thus $(\ell, m) \in\{(2,3),(2,4),(3,3)\}$. Finally, if $p=1$ and $m=2$, then $\ell>2$ and the crossing number is $2 \ell+2$. That is, $\ell \in\{3,4\}$.

Once having enumerated the $K(\ell, m, n, p)$ with at most 11 crossings and written them in the form .a.b.c, we can now locate them in the tables. To do this we use the symmetries given by Corollaries 10.3 and 10.6 (note that if $t=\left[a_{1}, \ldots, a_{n}\right]$ is rational then $\left.t 0=\left[a_{1}, a_{2}, \ldots, a_{n}, 0\right]\right)$.

This completes the proof of Theorem 10.10 .

Of the knots listed in Conway's tables in the form .a.b.c.d (or .a.b, or .a.b.c) that are not EM-knots, there are: 1 with 8 crossings, 3 with 9 crossings, 13 with 10 crossings, and 45 with 11 crossings. By Theorem 10.9 these all have unknotting number greater than 1 .

Next we consider the knots of the form $(a, b)(c, d)$ in Conway's notation. For these we first have the following result.

Theorem 10.11 Let $K=(a, b)(c, d)$, where $|a|,|b|,|c|$ and $|d|$ are $>1$ and either $a b>0$ or $c d>0$. Then $K$ does not have unknotting number 1 .

Proof It is easy to see (possibly using Lemma 2.2) that $(a, b)(c, d)=\mathcal{S}\left(-\frac{1}{a},-\frac{1}{b}\right.$; $\left.\frac{1}{c}, \frac{1}{d}\right)$. It follows from Theorem 9.8 that $K$ has unknotting number 1 if and only if $K$ is an EM-knot. Now by Corollary 3.2, the EM-knots are all of the form $\mathcal{S}(\alpha, \beta ; \gamma, \delta)$ with $|\alpha|,|\beta|,|\gamma|,|\delta|<1$ and $\alpha \beta<0, \gamma \delta<0$. Moreover, it is easy to verify, using Lemma 2.2, that if $\mathcal{S}(\alpha, \beta ; \gamma, \delta)= \pm \mathcal{S}\left(\alpha^{\prime}, \beta^{\prime} ; \gamma^{\prime}, \delta^{\prime}\right)$, where $|\alpha|,\left|\alpha^{\prime}\right|$, etc. are all $<1$, and $\alpha \beta<0$ and $\gamma \delta<0$, then $\alpha^{\prime} \beta^{\prime}<0$ and $\gamma^{\prime} \delta^{\prime}<0$. It follows that a knot $K$ of the form described in the theorem is never an EM-knot.

In Conway's tables, there are 48 knots listed in the form $(a, b)(c, d): 310-$ crossing alternating knots, 7 10-crossing non-alternating knots, 10 11-crossing alternating knots, and 28 11-crossing non-alternating knots.

Theorem 10.12 Up to 11 crossings, of the 48 knots listed in Conway's tables as $(a, b)(c, d)$, the only ones with unknotting number 1 are the four non-alternating 11crossing knots $(3,2+)(21,2-),(21,2+)(21,2-),(3,2+)-(21,2)$, and $(21,2+)-$ $(21,2)$. 
Proof All the knots in question satisfy the hypotheses of Theorem 10.11 except those with $(a, b)=(3,2+)$ or $(21,2+)$. Of these, the four listed in the theorem are easily seen to have unknotting number 1 . The others have $(c, d)=(3,2),(21,2),(3,2-)$ or $-(3,2)$. From now on, let $K$ be a knot of the form $(a, b)(c, d)$ where $(a, b)$ (resp. $(c, d)$ ) is one of the two (resp. four) possibilities listed. Recall (see proof of Theorem 10.11) that $K=\mathcal{S}\left(-\frac{1}{a},-\frac{1}{b} ; \frac{1}{c}, \frac{1}{d}\right)$.

First note that $K$ is not an EM-knot. For $\left\{\frac{1}{a}, \frac{1}{b}\right\}=\left\{\frac{1}{3}, \frac{3}{2}\right\}$ or $\left\{\frac{2}{3}, \frac{3}{2}\right\}$, and it follows easily from Lemma 2.2 that $K$ is not of the form $\mathcal{S}(\alpha, \beta ; \gamma, \delta)$ with $|\alpha|,|\beta|,|\gamma|,|\delta|<1$, and hence not an EM-knot by Corollary 3.2.

By Theorem 6.2, if $K$ has unknotting number 1 then it can be unknotted by a crossing move in $\mathcal{M}\left(-\frac{1}{a},-\frac{1}{b}\right)$ or $\mathcal{M}\left(\frac{1}{c}, \frac{1}{d}\right)$. Since $|c|,|d|>1$, the former is impossible, by Corollary 9.7. So the unknotting move is contained in $\mathcal{M}\left(\frac{1}{c}, \frac{1}{d}\right)$, transforming $\mathcal{M}\left(\frac{1}{c}, \frac{1}{d}\right)$ to a tangle $\mathcal{T}$ that unknots $\mathcal{M}\left(-\frac{1}{a},-\frac{1}{b}\right)=\mathcal{M}\left(-\frac{1}{3},-\frac{3}{2}\right)$ or $\mathcal{M}\left(-\frac{2}{3},-\frac{3}{2}\right)$. By Lemma 9.5, we see that in both cases $\mathcal{T}=\mathcal{R}(-1 / 2)$.

Consider the case $(c, d)=(3,2)$. Then $\mathcal{M}(\gamma, \delta)=\mathcal{M}\left(\frac{1}{3}, \frac{1}{2}\right)$. By Corollary 9.3, this can be transformed to $\mathcal{R}(-1 / 2)$ by a crossing move if and only if there is an integer $k$ such that $k \cdot 2+1=-1$, and $1 / 3=k+\frac{2 s^{2}}{2 r s \pm 1}$ for some $s \geq 1,(r, s)=1$. The first equation gives $k=-1$, and the second now gives $\pm 2 s^{2}=1-3 k=4$, which is impossible.

The other three cases $(c, d)=(21,2),(3,2-)$ and $-(3,2)$ are similar. We omit the details.

We now consider the knots of the form $. a .(b, c)$ or $.(b, c) . a$. First we have the following, which is an immediate consequence of Theorem 10.1 .

Lemma $10.13 \quad . a .(b, c)$ and -. $(b, c) . a$ are mutants.

Lemma 10.13 and Theorem 7.1 imply that $. a .(b, c)$ has unknotting number 1 if and only if . $(b, c) . a$ does, so we restrict attention to knots of the first type.

Theorem 10.14 Suppose that $a>0$ and $|b|,|c|>1$. Then $. a .(b, c)$ has unknotting number 1 if and only if $\Delta\left(\frac{a}{a+1}, \frac{1}{2}\right)=\Delta\left(-\frac{1}{b}, \frac{c+1}{c}\right)=1$.

Proof The knot $. x . y$ has the form shown in Figure 10.5. Therefore $K=. a .(b, c)$ is of the form $\mathcal{M}(\alpha, \beta) \cup \mathcal{M}(\gamma, \delta) \cup \mathcal{M}(-1 / 2, *)$, where $\alpha=a /(a+1), \beta=-1 / 2$, $\gamma=-1 / b$, and $\delta=-(c+1) / c$; see Figure 10.6. Note that the conditions on $a, b$ and 


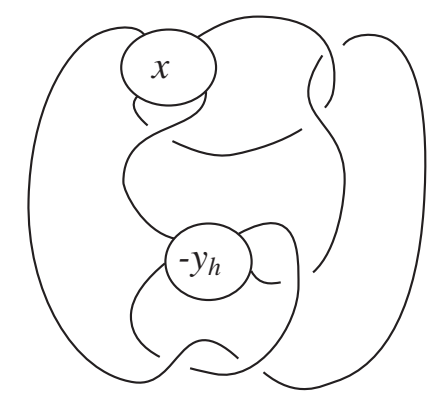

Figure 10.5

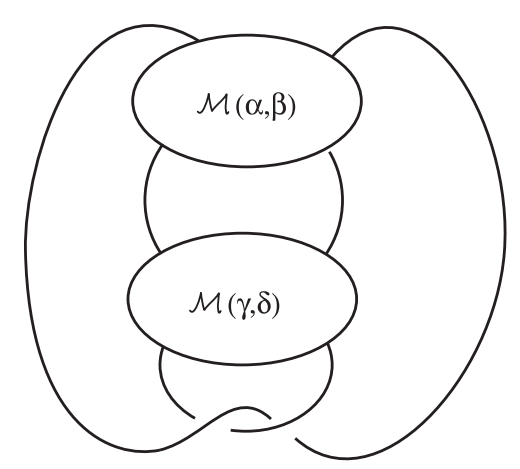

Figure 10.6

$c$ guarantee that $\alpha, \beta, \gamma, \delta \in \mathbb{Q}-\mathbb{Z}$. This is a decomposition of $K$ into elementary tangles.

Since $K$ does not have a unique essential Conway sphere, $K$ is not an EM-knot. Hence, if $u(K)=1$, then either conclusion (1) or (3) of Theorem 8.2 holds.

First, suppose (1)(a) of Theorem 8.2 holds for $\mathcal{M}(\alpha, \beta)$. By Lemma 9.2(3), $\mathcal{M}(\alpha, \beta)$ is transformed to a rational tangle $\mathcal{R}\left(p^{\prime} / q\right)$ with $q \geq 2$. If this unknots $K$, then $\mathcal{R}\left(p^{\prime} / q\right) \cup \mathcal{M}(-1 / 2, *)$ must be an integral tangle; see Figure 10.7. Hence $p^{\prime}= \pm 1$, and $\mathcal{R}\left(p^{\prime} / q\right) \cup \mathcal{M}(-1 / 2, *)=\mathcal{R}\left(\frac{2}{1 \pm 2 q}\right)$. Therefore $q=0$ or \pm 1 , a contradiction. Exactly the same argument shows that (1)(a) of Theorem 8.2 does not hold for $\mathcal{M}(\gamma, \delta)$.

Next suppose that we have conclusion (3) of Theorem 8.2 and that (1) of that theorem does not hold. By the remark after Theorem 8.2, for $\mathcal{M}=\mathcal{M}(\alpha, \beta)$ or $\mathcal{M}(\gamma, \delta)$, and $\mathcal{M}^{\prime}=\mathcal{M}(\gamma, \delta)$ or $\mathcal{M}(\alpha, \beta)$, respectively, we have $\mathcal{M} \cup \mathcal{M}(-1 / 2, *)=\mathcal{A}$, an EM-tangle, and the rational tangle $\mathcal{R}$ resulting from the standard unknotting move in $\mathcal{A}$ must unknot $\mathcal{M}^{\prime}$. Hence $\mathcal{R}$ must be an integral tangle, $\mathcal{R}(r / 1)$. Here $r= \pm \Delta(\alpha, \beta)$ where $\alpha$ is the tangle slope corresponding to $\mathcal{R}$ and $\beta$ is the tangle slope of the Conway 


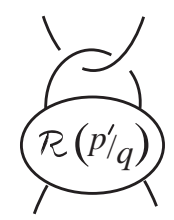

Figure 10.7

disk of $\mathcal{A}$. From Figure 4.3, we determine that $r= \pm 4 m$ when $\mathcal{A}=\mathcal{A}_{1}(\ell, m)$ (the rational tangle is $\left.\mathcal{R}\left(\frac{1-2 m}{4 m}\right)\right)$ and that $r= \pm(\ell(1-2 m)+2)$ (the rational tangle is $\left.\mathcal{R}\left(\frac{2 m-1}{\ell(1-2 m)+2}\right)\right)$ when $\mathcal{A}=\mathcal{A}_{1}(\ell, m)$.

Since $|\alpha|,|\beta|<1$, it follows from Lemma 9.5(2) that $\mathcal{M}=\mathcal{M}(\alpha, \beta), \mathcal{M}^{\prime}=\mathcal{M}(\gamma, \delta)$. Let $-\frac{1}{b}=\frac{r_{1}}{t_{1}},-\frac{1}{c}=\frac{r_{2}}{t_{2}}$ where $r_{i}, t_{i} \in \mathbb{Z}$ and $t_{i}>1$. Applying Lemma 9.5(1) with $x=r$ we get that $r-\frac{1}{b}-\frac{1}{c}-1=\frac{ \pm 1}{t_{1} t_{2}}$. As $|b|,|c|, t_{1}, t_{2}>1,|r|<4$. From the preceding paragraph, this implies that when $\mathcal{A}=\mathcal{A}_{1}(\ell, m),|4 m|<4$. But $m \neq 0$, a contradiction. When $\mathcal{A}=\mathcal{A}_{2}(\ell, m),|\ell(1-2 m)+2|<4$. Thus $|\ell||1-2 m|<6$. Since $|\ell| \geq 2$ and $m \notin\{0,1\}$, this is again a contradiction. Thus conclusion (3) of Theorem 8.2 cannot hold.

We conclude that (1)(b) of Theorem 8.2 must hold. The crossing move transforms $\mathcal{M}\left(-\frac{1}{2}, *\right)$ to $\mathcal{M}\left(\frac{1}{0}, *\right)$, which transforms $K$ to the connected sum of $1^{*} \mathcal{M}(\alpha, \beta)$ and $1^{*} \mathcal{M}(\gamma, \delta)$. The result now follows from Lemma 9.4.

In Conway's tables, there are 22 knots listed in the form $. a .(b, c)$ or $.(b, c) . a: 811-$ crossing alternating knots and $1411-$ crossing non-alternating knots. (They come in mutant pairs $. a .(b, c)$ and $.(b, c) . a$.) They all have $a=2$ or $20=1 / 2$, the alternating knots have $(b, c)=(3,2)$ or $(21,2)$, while the non-alternating knots have $(b, c)=$ $(3,2-),(21,2-),-(3,2)$ or $-(21,2)$.

Theorem 10.15 Of the knots listed in Conway's tables of the form .a.(b,c) or . $(b, c) . a$, those with unknotting number 1 are precisely the 611 -crossing non-alternating knots with $(b, c)=-(3,2)$ or $-(21,2)$.

Proof This follows easily from Theorem 10.14.

Finally, we consider the three knots in Conway's tables of the form $(a, b) 1(c, d)$. First we have the following lemma.

Lemma 10.16 If $a, b, c, d>1$ then $(a, b) 1(c, d)$ is not an EM-knot. 
Proof The knot $(a, b) 1(c, d)$ has the form shown in Figure 10.8. Consider the arc that joins the SE-corners of the two Montesinos tangles. By swinging this arc over the right-hand tangle, one sees that $(a, b) 1(c, d)=\mathcal{S}(1 / a, 1 / b+1 ; 1 / c, 1 / d+1)$. By Lemma 2.2, it is easy to see that this is never of the form $\mathcal{S}(\alpha, \beta ; \gamma, \delta)$ with $|\alpha|,|\beta|,|\gamma|,|\delta|<1$. But the EM-knots are all of this form, by Lemma 3.1.

Theorem 10.17 The 11-crossing alternating knots $(3,2) 1(3,2),(3,2) 1(21,2)$ and $(21,2) 1(21,2)$ do not have unknotting number 1.

Proof Let $K=(a, b) 1(c, d)$. Note that for the knots under consideration, $\frac{1}{a}, \frac{1}{b}, \frac{1}{c}, \frac{1}{d} \in$ $\mathbb{Q}-\mathbb{Z}$. Clearly $K$ does not contain an EM-tangle. Also, it is not an EM-knot by Lemma 10.16.

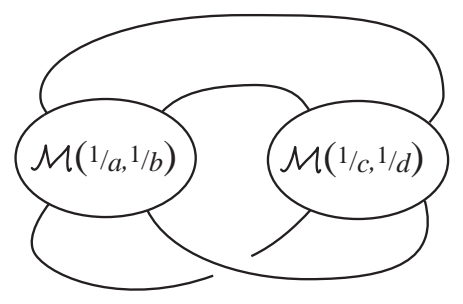

Figure 10.8

Hence, by Theorem 8.2, if $u(K)=1$ then the unknotting move takes place in a rational substituent of $\mathcal{M}\left(\frac{1}{a}, \frac{1}{b}\right)$ or $\mathcal{M}\left(\frac{1}{c}, \frac{1}{d}\right)$, transforming it to a rational tangle $\mathcal{R}(r)$. By symmetry we may assume that the unknotting move takes place in $\mathcal{M}\left(\frac{1}{a}, \frac{1}{b}\right)$. By Lemma 9.5(1), the tangle $\mathcal{R}(r)$ must be an integral tangle $\mathcal{R}(m)$. It follows that $r=[m,-1,0]=\frac{m}{1-m}$. By Lemma 9.5(2), since $\left|\frac{1}{c}\right|,\left|\frac{1}{d}\right|<1$, we must have $|m| \leq 1$. On the other hand, by Lemma 9.2(3), there exists an integer $k$ such that $\frac{k q+p}{q}=r=\frac{m}{1-m}$, where $p / q=1 / a$ or $1 / b$. Since $q \geq 2$ and $(p, q)=1, m=0$ or 1 is impossible. Hence $m=-1$ and $q=2$. Therefore $p / q=1 / 2=1 / a$ (since $(a, b)=(3,2)$ or $(21,2)$ ), and $2 k+1=m=-1$, giving $k=-1$. Also, by Lemma 9.2(4), the other rational substituent of $\mathcal{M}\left(\frac{1}{a}, \frac{1}{b}\right)$, namely $1 / b$, must satisfy $\frac{1}{b}=-1+\frac{2 s^{2}}{2 r s \pm 1}, s \geq 1$, $(r, s)=1$. But in both cases, $b=3$ and $b=21=3 / 2$, this is easily seen to be impossible.

Remark For knots of up to 10 crossings, until the work described here and the work of Ozsváth and Szabó [27], there were 41 knots for which it was not known whether or not they had unknotting number 1 . We ruled out 14 of them (see above): the two 
9-crossing knots $9_{29}=.2 .20 .2$ and $9_{32}=.21 .20$, and the twelve 10 -crossing knots $10_{79}=(3,2)(3,2), 10_{81}=(21,2)(21,2), 10_{83}=.31 .2,10_{86}=.31 .20,10_{87}=.22 .20$, $10_{90}=.3 .2 .2,10_{93}=.3 .20 .2,10_{94}=.30 .2 .2,10_{96}=.2 .21 .2,10_{148}=(3,2)(3,2-)$, $10_{151}=(21,2)(21,2-)$, and $10_{153}=(3,2)-(21,2)$. Meanwhile, Ozsváth and Szabó [27], using their remarkable Heegaard Floer homology theory, ruled out all 41 knots except $10_{153}$. So the knots with 10 or fewer crossings and unknotting number 1 are completely determined.

\section{Deciding if a large algebraic knot has unknotting num- ber 1}

It is unknown if the unknotting number of a knot is a computable invariant. Even the following special case is open:

Question 11.1 Is there an algorithm to decide whether or not a given knot has unknotting number 1 ?

Note that by Haken [12] there is an algorithm to decide if a knot has unknotting number 0 .

Theorem 8.2 allows us to answer Question 11.1 affirmatively for large algebraic knots.

Theorem 11.2 There is an algorithm to decide whether or not a given large algebraic knot $K$, described as a union of elementary marked tangles (Figure 8.1) and 4-braids in $S^{2} \times[0,1]$, has unknotting number 1 , and, if so, to identify an unknotting crossing move.

Proof Note that none of the rational tangles in a constituent elementary tangle of $K$ is integral or $\mathcal{R}(1 / 0)$ (that is, the distance between the slope of such a rational tangle and the orbifold $S^{1}$-fiber of the elementary tangle is at least two).

Definition Let $\mathcal{T}$ be a marked tangle in the 3-ball. The number $p / q \in \mathbb{Q} \cup\{\infty\}$ is called an unknotting slope for $\mathcal{T}$ if $\mathcal{T} \cup \mathcal{R}(-p / q)$ (ie $1^{*}(\mathcal{T}+\mathcal{R}(-p / q))$ ) is the unknot.

Remark As long as $\mathcal{T}$ is not rational, an unknotting slope for $\mathcal{T}$, if there is one, is unique. This follows, for example, from the fact that knots are determined by their complements and the $\mathbb{Z}_{2}-$ Smith Conjecture (applied to the double branched covers). 
Lemma 11.3 There is an algorithm to decide whether or not a given algebraic tangle (ie a union of elementary tangles and 4-braids) has an unknotting slope, and, if so, to find it.

Proof Again we assume the tangle is given as a union of elementary marked tangles and 4-braids in $S^{2} \times[0,1]$. Begin with the innermost, constituent, elementary tangles, necessarily of type I, and compute their unknotting slopes (if they exist) via Lemma 9.5(1). Then work outward along elementary tangles of type II determining unknotting slopes at each step. This is equivalent to solving equations of the following form: given $\frac{r_{1}}{s_{1}}, \frac{r_{2}}{s_{2}}$ find a rational $\frac{x}{y}$ such that $\frac{x}{y}+\frac{r_{1}}{s_{1}}=\frac{r_{2}}{s_{2}}$. In working outward, if one comes to a constituent tangle of type III, the corresponding unknotting slope (if it exists) must be the slope of the orbifold $S^{1}$-fiber.

Working outward in this way, either one finds at some point that there is no unknotting slope, in which case there is none for the algebraic tangle, $\mathcal{T}$, or one determines the unknotting slope for $\mathcal{T}$.

By Theorem 8.2, $K$ has unknotting number 1 if and only if one of the following options holds: (A) $K$ is an EM-knot; (B) $K$ contains an EM-tangle for which the standard crossing change unknots $K$; or (C) $K$ unknots by replacing a rational tangle in a constituent elementary tangle of type I or II with another rational tangle as described in Theorem 8.2(1).

We show that these options can be checked algorithmically.

(A) To see if $K$ is an EM-knot, first check that there are two elementary tangles of type I whose union is $K$. If so, compute the orders of the exceptional orbifold $S^{1}$-fibers of each elementary tangle and list the finite number of EM-knots having exceptional fibers of the same order. Check if $K$ is equivalent to one of these (eg, check that the orbifold $S^{1}$-fibers of the two elementary tangles intersect twice at the unique Conway sphere [ie, that the distance between the slopes of these fibers is 1 on the Conway sphere]. If so then $K$ can be written in the form $\mathcal{S}(\alpha, \beta ; \gamma, \delta)$ and Lemma 2.2 may be applied).

(B) To check the unknotting of $K$ by a standard crossing change in an EM-tangle, we list all pairs $\left\{X_{1}, X_{2}\right\}$ of a type I and type II elementary tangle of $K$ that share a common Conway sphere. The union $X_{1} \cup X_{2}$ is a candidate for an EM-tangle. Let $-\frac{p}{q}$ be the unknotting slope of the complementary tangle $\left(S^{3}, K\right)-\left(X_{1} \cup X_{2}\right)$. List the finitely many EM-tangles that have exceptional orbifold $S^{1}$-fibers of the same order as $X_{1} \cup X_{2}$. Then check if there is a homeomorphism from the candidate $X_{1} \cup X_{2}$ to one of these EM-tangles taking the slope $\frac{p}{q}$ to the slope of the rational tangle that results 
from the standard crossing change. (For example, check that the orbifold $S^{1}$-fibers of $X_{1}$ and $X_{2}$ intersect twice along the common Conway sphere. If so, $X_{1} \cup X_{2}$ may be rewritten in the form $\mathcal{S}\left(*, \frac{p_{1}}{q_{1}} ; \frac{p_{2}}{q_{2}}, \frac{p_{3}}{q_{3}}\right)$. Then check that

$$
\frac{p_{1}}{q_{1}}+n_{1}=\frac{v_{1}}{w_{1}}, \quad \frac{p_{2}}{q_{2}}+n_{2}=\frac{v_{2}}{w_{2}}, \quad \frac{p_{3}}{q_{3}}-n_{2}=\frac{v_{3}}{w_{3}}
$$

for some $n_{1}, n_{2} \in \mathbb{Z}$, where $\mathcal{A}_{\varepsilon}(\ell, m)=\mathcal{S}\left(*, \frac{v_{1}}{w_{1}} ; \frac{v_{2}}{w_{2}}, \frac{v_{3}}{w_{3}}\right)$. If so, there is a unique homeomorphism up to isotopy taking $X_{1} \cup X_{2}$ to $\mathcal{A}_{\varepsilon}(\ell, m)$. One then checks that $\frac{p}{q}$ is identified with the slope of the rational tangle gotten by the standard crossing move on $\mathcal{A}_{\varepsilon}(\ell, m)$.)

(C) To check the condition of Theorem 8.2(1), we check each constituent elementary tangle of type I or II as follows. For any elementary tangle of type II, we replace the rational tangle $\mathcal{R}(p / q)$ with $\mathcal{R}(1 / 0)$. If this yields the unknot (which can be checked algorithmically), then we check that $\frac{p}{q}=\frac{2 r s \pm 1}{2 s^{2}}$ for some $(r, s)=1$. If so one identifies the unknotting crossing move of $K$ as described, for example, in Lemma 8.1 (or Lemma 12.3).

Consider a constituent elementary tangle, $X$, of type I, and let $-\frac{v}{w}$ be the unknotting slope for the complementary tangle $\left(S^{3}-K\right)-X$ (if there is none, $K$ cannot be unknotted in this way). Decide if replacing some rational tangle, $\mathcal{R}(p / q)$, of $X$ by an integer tangle, $\mathcal{R}(k)$, changes $X$ to the rational tangle $\mathcal{R}(v / w)$. If so, determine if $\frac{p}{q}=\frac{2 s^{2}}{2 r s \pm 1}+k$ for some $(r, s)=1$. If so, then a variation of Lemma 8.1 will determine an unknotting crossing move for $K$ (see also Lemma 12.4).

\section{Unknotting in a minimal diagram}

In [18], Kohn made the following conjecture, which he showed was true for 2-bridge knots and links.

Conjecture (Kohn) Let $K$ be a knot or link with $u(K)=1$. There is a crossing in a minimal diagram of $K$ which, when changed, unknots $K$.

Note that the analog for knots with $u(K)>1$ is false [1], [26].

We shall show that the conjecture is true for alternating large algebraic knots.

From [32], we take the following

Definition A tangle diagram $D$ in a disk $\Delta$ is prime iff

(i) the underlying projection of $D$ is a connected subset of the disk $\Delta$ 
(ii) if $C$ is a circle in $\Delta$ meeting $D$ transversely in two points, then these points belong to the same edge of $D$ (ie, diagrammatic connected sums are not allowed).

Proposition 12.1 (Flyping conjecture for alternating tangles in a 3-ball) If $D, D^{\prime}$ are prime, alternating diagrams of the same marked tangle in a 3-ball, then $D$ and $D^{\prime}$ differ by a sequence of flypes.

Proof As outlined in [32, page 333] or [34, page 998], this follows from the rigid vertex version of the Flyping Conjecture proved in [22].

Definition A tangle $(B, T)$ in a 3-ball $B$ is prime iff

(i) $(B, T)$ is not rational;

(ii) if $S$ is a 2-sphere in $B-T$, then the 3-ball bounded by $S$ does not meet $T$;

(iii) if a 2-sphere $S$ in $B$ meets $T$ transversely in two points, then the 3-ball in $B$ bounded by $S$ meets $T$ in an unknotted arc.

Corollary 12.2 If $D, D^{\prime}$ are alternating diagrams of the same marked tangle in a 3-ball which is either prime or rational but not $\mathcal{R}(1 / 0), \mathcal{R}(0 / 1)$, then any (marked) tangle obtained by changing a crossing in $D$ can be gotten by changing a crossing in $D^{\prime}$.

Proof of Corollary 12.2 The hypotheses guarantee that if $D$ is not a prime diagram, then it has a nugatory crossing. (A circle violating (ii) of primeness of the diagram must encircle an alternating diagram of the unknot. This must have a nugatory crossing by the minimality of crossing number for reduced, alternating diagrams of links.) Thus by reducing all nugatory crossings in $D$, we leave a prime, alternating diagram. Similarly for $D^{\prime}$. Thus $D, D^{\prime}$ are related by a sequence of flypes and nugatory crossing reductions or creations. One checks that changing a crossing commutes with these operations.

Recall (Lemma 8.1) that $\mathcal{R}(p / q)$ can be transformed to $\mathcal{R}(1 / 0)$ by a crossing move if and only if there are coprime integers $r, s$ such that $p / q=\frac{2 r s \pm 1}{2 s^{2}}$.

Lemma 12.3 If $p / q=\frac{2 r s \pm 1}{2 s^{2}}$, where $(r, s)=1$ and $s \neq 0$, then $\mathcal{R}(p / q)$ can be transformed to $\mathcal{R}(1 / 0)$ by a crossing change in any alternating diagram for $\mathcal{R}(p / q)$. 


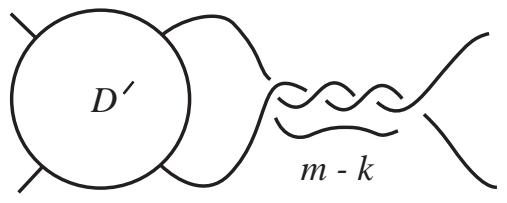

Figure 12.1

Proof By [18] the condition on $p / q$ is equivalent to the condition that $\pm p / q=$

$$
\begin{aligned}
& {\left[c_{1}, \ldots, c_{\ell-1}, c_{\ell}, 1,1, c_{\ell}-1, c_{\ell-1}, \ldots, c_{1}, c_{0}\right] \text { or }} \\
& {\left[c_{1}, \ldots, c_{\ell-1}, c_{\ell}-1,1,1, c_{\ell}, c_{\ell-1}, \ldots, c_{1}, c_{0}\right]}
\end{aligned}
$$

where $c_{i} \geq 1,0 \leq i \leq \ell-1, c_{\ell} \geq 2$. The corresponding diagram of $\mathcal{R}(p / q)$ is alternating, and the crossing change is visible in that diagram. By Corollary 12.2, the transformation $\mathcal{R}(p / q) \mapsto \mathcal{R}(1 / 0)$ can be effected by a crossing change in any alternating diagram.

Lemma 12.4 If $p / q \in \mathbb{Q}-\mathbb{Z}$, and $\mathcal{R}(p / q)$ can be transformed to $\mathcal{R}(k)$ by a crossing move, then it can be transformed to $\mathcal{R}(k)$ by a crossing change in any alternating diagram of $\mathcal{R}(p / q)$, unless $p / q= \pm[\ell, 2, m]$, where $\ell>0, m \geq 0$, and $k= \pm(\ell+$ $m+2)$.

Proof By Corollary 12.2, we only need exhibit a crossing change in some alternating diagram of $\mathcal{R}(p / q)$. If $k=0$ then the result holds by rotating and applying Lemms 8.1 and 12.3. So assume $k \neq 0$. We may also suppose, without loss of generality, that $p / q>0$. Write $p / q=p^{\prime} / q+m, 0<p^{\prime} / q<1, m \geq 0$. Then $\mathcal{R}(p / q-k)=$ $\mathcal{R}\left(p^{\prime} / q+(m-k)\right)$ can be transformed to $\mathcal{R}(0)$ by a crossing move.

Let $D^{\prime}$ be a positive alternating diagram of $\mathcal{R}\left(p^{\prime} / q\right)$ (see Figure 12.1). There is such a diagram since $\frac{p^{\prime}}{q}>0$.

\section{Case (1) $m-k \geq 0$}

In this case $\mathcal{R}(p / q-k)$ has the alternating diagram shown in Figure 12.1

By the case $k=0$ above, $\mathcal{R}(p / q-k)$ can be transformed to $\mathcal{R}(0)$ by changing a crossing $c$ in this diagram. Clearly $c$ must be a crossing of the diagram $D^{\prime}$ (eg, by computing the associated rational number after the crossing change). Let $D$ be the alternating diagram of $\mathcal{R}(p / q)=\mathcal{R}\left(p^{\prime} / q+m\right)$ obtained by putting $m$ horizontal $\frac{1}{2}$-twists on the right of $D^{\prime}$. Then changing the crossing $c$ in $D$ transforms $\mathcal{R}(p / q)$ to $\mathcal{R}(k)$. 


\section{Case (2) $m-k<0$}

Since $0<p^{\prime} / q<1$, the diagram $D^{\prime}$ ends up with $r \geq 1$ vertical $\frac{1}{2}$-twists; see Figure 12.2. Hence $\mathcal{R}(p / q-k)=\mathcal{R}\left(p^{\prime} / q+(m-k)\right)$ has the diagram $D_{0}$ shown in Figure 12.3 .

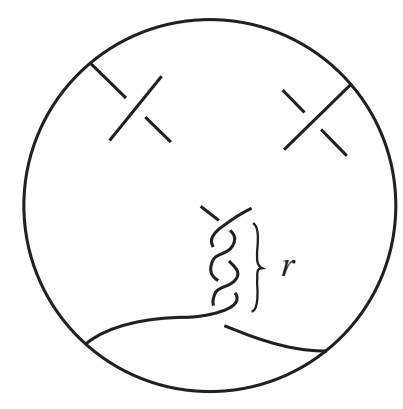

Figure 12.2

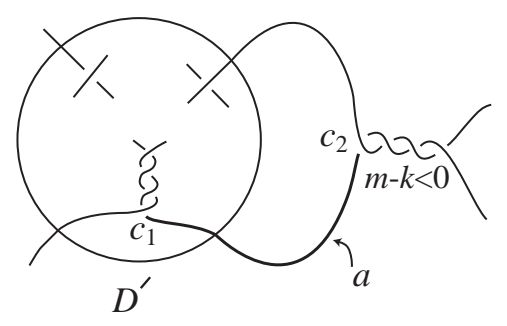

Figure 12.3

Let $a$ be the arc indicated by the bold line in Figure 12.3. Swinging $a$ underneath $D^{\prime}$ gives the diagram $D_{1}$ shown in Figure 12.4. Note that this is a (negative) alternating diagram. Therefore, by the case $k=0$ above, $\mathcal{R}(p / q-k)$ can be transformed to $\mathcal{R}(0)$ by changing some crossing $c$ in $D_{1}$. Clearly $c$ is either a crossing of $D^{\prime}$ (other than $c_{1}$ ) or the new crossing $c_{0}$.

In the first case, $\mathcal{R}\left(p^{\prime} / q+m\right)=\mathcal{R}(p / q)$ is transformed to $\mathcal{R}(k)$ by changing the same crossing $c$ in the alternating diagram $D$ of $\mathcal{R}\left(p^{\prime} / q+m\right)$ defined in Case (1).

In the second case, changing the crossing $c_{0}$ in $D_{1}$ and swinging the arc $a$ over $D^{\prime}$ clearly gives the same tangle as changing the crossings $c_{1}$ and $c_{2}$ in the diagram $D_{0}$. By hypothesis, this is $\mathcal{R}(0)$. Hence changing only $c_{1}$ in $D_{0}$ gives $\mathcal{R}(-2)$. Therefore $r \leq 2$ (else the crossing change would not yield an integral tangle). 


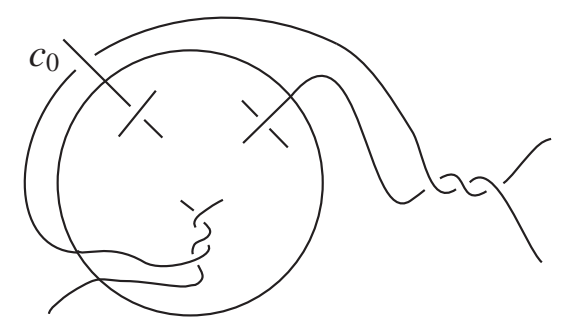

Figure 12.4

Claim If $r=1$, then $\frac{p}{q}=[\ell-1,1,1, m]$ where $\ell \geq 2, k=m+2-\ell$.

If $r=2$, then $\frac{p}{q}=[\ell, 2, m]$ where $\ell>0, k=m+2+\ell$.

Proof of Claim Assume $r=1$. The positive continued fraction expansion for the rational tangle of Figure 12.3 with crossing $c_{1}$ changed gives

$$
-2=(m-k)+\frac{1}{-1+\frac{s}{\ell}}=(m-k)+\frac{\ell}{s-\ell}
$$

where $0<\frac{s}{\ell}<1,(s, \ell)=1$. Integrality gives $s=\ell-1$ and

$$
\frac{p}{q}=m+\frac{1}{1+\frac{s}{\ell}}=m+\frac{1}{1+\frac{1}{1+\frac{1}{\ell-1}}}
$$

where $\ell-1>0$. Thus $\frac{p}{q}=[\ell-1,1,1, m]$. Furthermore

$$
-2=(m-k)+\frac{1}{-1+\frac{1}{1+\frac{1}{\ell-1}}}=m-k-\ell
$$

as required.

Similarly, if $r=2$ we have

$$
-2=(m-k)+\frac{1}{0+\frac{s}{\ell}}
$$

This implies that $s=1, \ell>0$. Then $-2=(m-k)+\ell$. Finally $\frac{p}{q}=[\ell, 2, m]$.

By the Claim, if $r=1$ then we are in Case (1). When $r=2$, we obtain the list of tangles stated in the Lemma.

Theorem 12.5 Let $K$ be an alternating large algebraic knot with unknotting number 1 . Then $K$ can be unknotted by a crossing change in any alternating diagram of $K$. 
Proof By [22], any two reduced alternating diagrams of $K$ are related by flype moves. It follows easily that if $K$ can be unknotted by a crossing change in some alternating diagram then it can be unknotted by a crossing change in any alternating diagram.

In what follows we use the notion of the "visibility" of a Conway sphere or disk in an alternating diagram as discussed in [33]. In particular, [21] shows that in an alternating diagram a Conway sphere is either visible, or hidden in a very specific way (see Figures 3(i), (ii) of [33]). In the latter case, there is a standard move on the diagram to make the sphere visible (see Figure 3(iii) of [33]). For a reduced alternating diagram of an elementary tangle, [33, page 326] shows that the arguments of [21] can also be used to say that the Conway disk must be visible.

First suppose that we are in Case (1) of Theorem 8.2.

Let $D$ be a reduced alternating diagram of $K$. Suppose we are in subcase (a), so that the unknotting crossing move takes place in a rational subtangle of an elementary tangle $\mathcal{T}$ of type I. Let $S$ be the boundary of $\mathcal{T}$, and suppose that $S$ is visible in $D$. Then after flyping if necessary (see [33, page 326] for the visibility of the Conway disk), we may assume that $D$ contains a subdiagram of the form shown in Figure 8.1(I). By Theorem 8.2, the crossing move transforms $\mathcal{R}(p / q)$ to $\mathcal{R}(k)$. Since $p / q \notin \mathbb{Z}$, it follows from Lemma 12.4 that this can be achieved by a crossing change in the diagram $D_{1}$, unless (without loss of generality) $p / q=[\ell, 2, m], \ell>0, m \geq 0$, and $k=\ell+m+2$. Since $k>0$ and $D$ is alternating, we see that replacing $D_{1}$ with the standard diagram of $\mathcal{R}(k)$ gives an alternating diagram $D^{\prime}$. Also, since $D$ is reduced and $S$ is essential, it is easy to see that $D^{\prime}$ is reduced. Hence $D^{\prime}$ is a diagram of a non-trivial knot, a contradiction.

Next suppose that we are in subcase (b) of Theorem 8.2, Case (1), and that the boundary components of the corresponding elementary tangle of type II are both visible in $D$ (Figure 8.1(II)). The crossing move transforms $\mathcal{R}(p / q)$ to $\mathcal{R}(1 / 0)$, and, by Proposition 12.1 and (the proof of) Lemma 8.1, this can be achieved by a crossing change in the diagram $D_{3}(q \neq 0)$.

It remains to consider (a) and (b) when the relevant Conway spheres are hidden in $D$. So suppose we are in subcase (a), and the boundary $S$ of the corresponding elementary tangle $\mathcal{T}$ of type I is hidden in $D$. Making $S$ visible as described in [33], we get a diagram in which the tangle $\mathcal{T}$ appears as in Figure 12.5. Note that the diagrams $D_{1}, D_{2}$ of $\mathcal{R}(r / s), \mathcal{R}(p / q)$ in Figure 12.5 will be alternating. Suppose, without loss of generality, that the unknotting crossing move takes place in the right-hand rational tangle $\mathcal{R}(p / q)$, transforming it to $\mathcal{R}(k)$ for some integer $k$.

We first argue that $k=0$ or 1 . Assume not. Let $\mathcal{R}(c / d)$ be the subtangle of $\mathcal{R}(r / s)$ encapsulated in the circle of $D_{1}$ of Figure 12.5. We then have the equation $\frac{d}{c}=1+\frac{s}{r}$. 


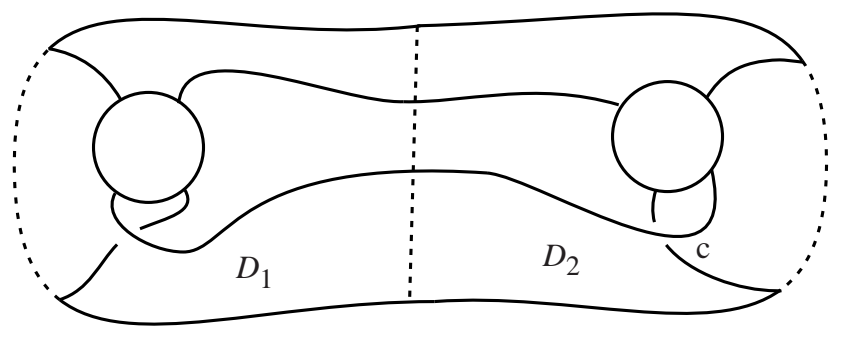

Figure 12.5: $\mathcal{T}=\mathcal{R}(r / s)+\mathcal{R}(p / q)$

Note that since the diagram of $\mathcal{R}(r / s)$ is alternating, $c / d<0$. Now the crossing move we are considering turns $\mathcal{T}$ into $\mathcal{R}\left(\frac{r}{s}+k\right)$. This has to be of the form $\mathcal{R}(1 / x), x \in \mathbb{Z}$, or $\mathcal{R}(0 / 1)$. (Write $K=\mathcal{T} \cup \mathcal{T}^{\prime}$, as in Figure 3(iii) of [33], where $\mathcal{T}^{\prime}$ is also of the form of Figure 12.5. Since the corresponding subdiagrams, $D_{i}^{\prime}$, of $\mathcal{T}^{\prime}$ are alternating, if either $D_{i}^{\prime}$ is a diagram of a rational tangle $\mathcal{R}(p / q)$, then $q>0$. Arguing on the level of double branched covers, as in Lemma 9.5(1), we see that $\Delta\left(\frac{r}{s}+k, \frac{0}{1}\right) \leq 1$. Hence $\frac{r}{s}+k=\frac{1}{x}$ or $\frac{0}{1}$.) Since $D_{1}$ in Figure 12.5 for $\mathcal{T}$ is alternating, $\left|\frac{r}{s}\right|<1$. Thus $\frac{r}{s}+k \neq \frac{0}{1}$. We assume $\frac{r}{s}+k=\frac{1}{x}, x \in \mathbb{Z}$. Then $\frac{r}{s}=\frac{1-k x}{x}$, hence $\frac{s}{r}=\frac{x}{1-k x}$. Therefore $0>\frac{d}{c}=1+\frac{x}{1-k x}=(1-(k-1) x) /(1-k x)$, implying that $k=0,1$.

By Lemma 12.4, since $\frac{p}{q} \notin \mathbb{Z}$ and $k=0,1$, we see that $\mathcal{R}(p / q)$ can be transformed to $\mathcal{R}(k)$ by a crossing change in the minimal diagram $D_{2}$. Now $D_{2}$ can be obtained by adding a vertical right-handed twist, given by the crossing marked $c$, to the diagram of the tangle $\mathcal{R}(a / b)$ which was visible in the original alternating diagram of $K$. If the crossing changed in $D_{2}$ is not $c$, then this is a crossing change in the original diagram. So suppose the crossing changed is $c$. Since this gives $\mathcal{R}(k)$, we see that $\mathcal{R}(p / q)$ $(\mathcal{R}(a / b)$, resp.) is gotten by adding two (one, resp.) right-handed vertical twists to $\mathcal{R}(k)$. Since $\frac{p}{q} \neq \frac{0}{1}$, we see that $k \neq 0$. Thus $k=1$ and $\frac{a}{b}=\frac{1}{2}, \frac{p}{q}=\frac{1}{3}$. But then one sees that the crossing change at $c$ can be accomplished by a crossing change in the diagram of $\mathcal{R}(a / b)$ by Corollary 12.2 . Thus $K$ can be unknotted by a crossing change in $D$.

To finish the proof of Theorem 12.5 in Case I, we consider the case when the crossing move is in an elementary tangle of type II where one of the boundary components, $S_{1}$, is hidden in $D$. Making $S_{1}$ visible gives a diagram containing a subdiagram as shown in Figure 12.6.

Lemma 12.6 Let $F_{1}$ be the disk pictured in Figure 12.6. Then $F_{1}$ is a Conway disk for $S_{1}$. Furthermore, any Conway disk for $S_{1}$ is parallel to $F_{1}$. 


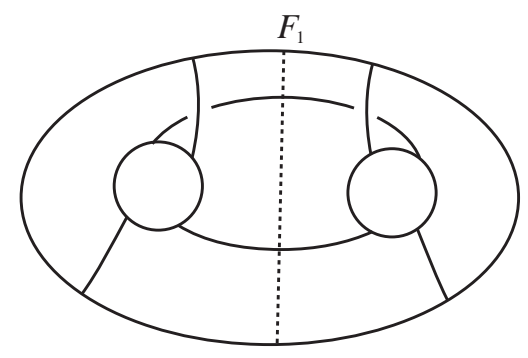

Figure 12.6

Proof By Corollary 3.3 of [33], $F_{1}$ is a Conway disk for the prime tangle bounded by $S_{1}$ in Figure 12.6. We assume for contradiction that there is a Conway disk, $F_{2}$, of $S_{1}$ that is not parallel to $F_{1}$. Then we may take $F_{2}$ to be disjoint from $F_{1}$. In particular, the slope of $F_{2}$ on $S_{1}$ is $\frac{1}{0}$ (in the diagram coordinates). Let $\mathcal{T}$ be the tangle containing $F_{2}$ after cutting Figure 12.6 along $F_{1}$. Without loss of generality assume this is the right-hand side of $F_{1}$. Then $\mathcal{T}$ is an alternating tangle for which $F_{2}$ is an essential Conway disk with slope $\frac{1}{0}\left(F_{2}\right.$ is not parallel to $\left.F_{1}\right)$. After possibly flyping, we can write $\mathcal{T}$ as the union of a positive braid in $S^{2} \times I$, with at least one vertical twist, and a reduced alternating tangle $\mathcal{T}^{\prime}$. See Figure 12.7.

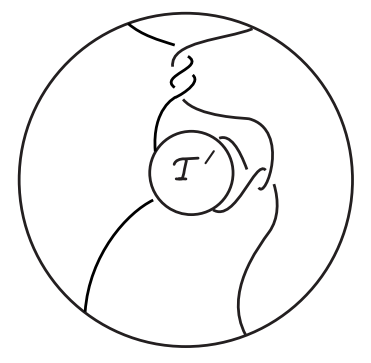

Figure 12.7

After an isotopy we may assume that $F_{2}$ intersects the boundary of $\mathcal{T}^{\prime}$ in a single circle, thereby writing $F_{2}$ as the union of an annulus in $S^{2} \times I$ and an essential Conway disk, $F_{2}^{\prime}$, in $\mathcal{T}^{\prime}$. By [33, page 326], $F_{2}^{\prime}$ can be taken to be visible, or hidden in a very special way. If visible, then its slope on $\partial \mathcal{T}^{\prime}$ (with coordinates from the diagram) is either $\frac{0}{1}$ or $\frac{1}{0}$. Since the braiding in $S^{2} \times I$ is positive with at least one vertical twist, this means the slope of $F_{2}$ on $\mathcal{T}$ cannot be $\frac{1}{0}$, a contradiction (note that the flyping did not change the slope of $F_{2}$ ).

So we assume $F_{2}^{\prime}$ is hidden in $\mathcal{T}^{\prime}$. But then [33] shows that $\mathcal{T}^{\prime}$ has a subdiagram as in Figure 12.8 and shows that the slope of $F_{2}^{\prime}$ on $\partial \mathcal{T}^{\prime}$ is either $\frac{0}{1}, \frac{1}{0}, \frac{1}{1}\left(-\frac{1}{1}\right.$ does not 


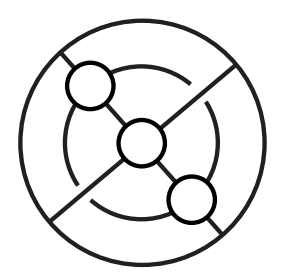

Figure 12.8

occur since the braiding is positive). Again, the fact that the braiding in $S^{2} \times I$ is positive with at least one vertical twist guarantees that the slope of $F_{2}$ on $\partial \mathcal{T}$ is not $\frac{1}{0}$ as we have assumed.

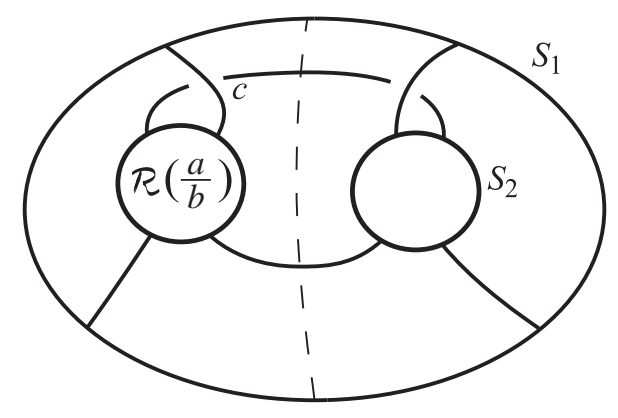

Figure 12.9

Lemma 12.6 allows us to say that, after isotoping to make $S_{1}$ visible, the elementary tangle of type II becomes visible as in Figure 12.9. In particular, we may assume the unknotting arc lies to the left of $F_{1}$ in this figure. By Theorem 8.2 and Lemma 12.3, this can be achieved by a crossing change in the (alternating) diagram on the left of Figure 12.9. If the crossing changed is not $c$, then this crossing change corresponds to a crossing change in the original diagram $D$. If the crossing changed is $c$, then $a / b=-1$. As argued above, changing the crossing $c$ is equivalent to changing the single crossing in $\mathcal{R}(-1)$, which corresponds to a crossing in $D$.

This finishes the proof of Theorem 12.5 in Case (1). Cases (2) and (3) of the theorem are proved in Theorems 12.7 and 12.8 .

Theorem 12.7 Let $K$ be an EM-knot. Then $K$ can be unknotted by a crossing change in any alternating diagram of $K$.

Proof Again by the Flyping Conjecture [22], we need only show that $K$ can be unknotted in some alternating diagram. Let $K=K(\ell, m, n, p)$. By [6, Proposition 1.4], 
we may assume, by taking the mirror-image of $K$ if necessary, that $\ell>1$. Lemmas 10.7 and 10.8 imply that $K=. a . b . c=\mathcal{S}\left(\frac{-1}{c+1}, \frac{a}{a+1} ; \frac{1}{b+1}, \frac{-1}{2}\right)$, where $a, b, c>0$. Thus $K$ has a diagram of the form shown in Figure 12.10. The unknotting arc $a_{0}$ as well as the

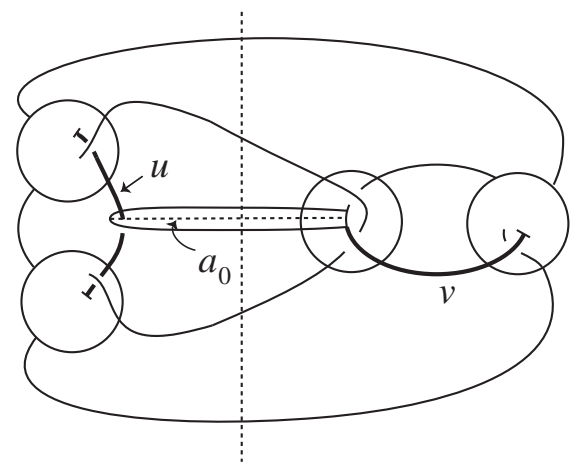

Figure 12.10

corresponding crossing move that unknots $K$ are shown. Let $u$ and $v$ be the arcs of the diagram indicated by the bold lines. Swinging $u$ "under" and $v$ "over" gives the diagram shown in Figure 12.11. This diagram is alternating since $a, b, c>0$. Also,

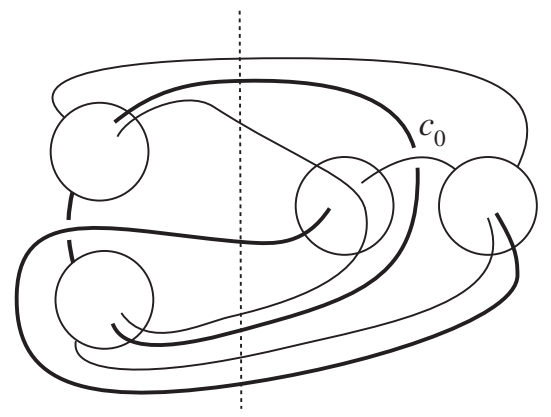

Figure 12.11

changing the crossing $c_{0}$ shown in Figure 12.11 has the same effect as performing the crossing move shown in Figure 12.10.

Theorem 12.8 Let $K$ be an alternating algebraic knot containing an EM-tangle whose boundary is an essential Conway sphere. If $u(K)=1$, then $K$ can be unknotted by a crossing change in any alternating diagram of $K$.

Proof Again, by [22] we need only verify this for some alternating diagram. Let $K$ be the union of essential tangles $\mathcal{P} \cup \mathcal{P}_{0}$ where $\mathcal{P}_{0}$ is an EM-tangle $\mathcal{A}_{\varepsilon}(\ell, m)$. Applying 
Theorem 6.2, we are either in Case (1) or (3). In Case (1), the proof of Theorem 12.5 guarantees the existence of an unknotting crossing change in an alternating diagram. Thus we assume we are in Case (3).

Lemma 12.9 In an alternating diagram of $K, \partial \mathcal{P}_{0}$ must be visible.

Proof Assume not. Then there is a diagram of $\mathcal{P}_{0}$ as in Figure 12.9. By [33], the disk $F_{1}$ in that figure is a Conway disk for $\mathcal{P}_{0}$. Thus it is the unique Conway disk for $\mathcal{P}_{0}$. Each side of $F_{1}$ is an alternating tangle. But this contradicts the fact that capping $\mathcal{A}_{\varepsilon}(\ell, m)$ along slope $\frac{1}{0}$ (ie, taking the denominator closure) gives either the unknot or Hopf link (by inspection of Figure 4.3).

Thus $\partial \mathcal{P}_{0}$ is visible and let $D_{0}$ be the corresponding subdiagram. This allows us to regard $\mathcal{P}_{0}$ as a marked tangle. By Corollary 12.2 we need to find some alternating diagram of this marked tangle that exhibits a crossing change which unknots $K$.

Theorem 6.2(3) gives an (unmarked) tangle homeomorphism $h: \mathcal{P}_{0} \rightarrow \mathcal{A}_{\varepsilon}(\ell, m)$ $=\mathcal{T}_{\varepsilon}(\ell, m)(1 / 2)$ which identifies the standard crossing move on $\mathcal{A}_{\varepsilon}(\ell, m)$ as an unknotting, crossing move for $K$. After possibly rotating, reflecting, or applying a mutation involution to $\mathcal{A}_{\varepsilon}(\ell, m)$, there is an alternating braided tangle $\mathcal{C}$ in $S^{2} \times I$ such that extending $\mathcal{A}_{\varepsilon}(\ell, m)$ by adjoining $\mathcal{C}$ gives a marked tangle $\mathcal{A}_{\varepsilon}(\ell, m) \cup \mathcal{C}$ which is equivalent to $\mathcal{P}_{0}$ as a marked tangle - via an extension of $h$.

Definition Let $E$ be the diagram in a disk $\Delta$ of a tangle in a 3-ball. A crossing $c$ of $E$ is said to be inessential iff there is a properly embedded arc in $\Delta$ that intersects $E$ only in $c$, dividing the four arcs of $E$ at $c$ into pairs. A diagram is reduced iff it contains no inessential crossings.

Figure 12.12 displays a reduced, prime, alternating diagram, $E$, for $\mathcal{A}_{\varepsilon}(\ell, m)$ which exhibits a crossing change sending $\mathcal{A}_{\ell}(\ell, m)$ to the rational tangle $\mathcal{T}_{\varepsilon}(\ell, m)\left(\frac{1}{0}\right)$. Letting $C$ denote an alternating braided diagram of the braided tangle $\mathcal{C}$, we have that $E \cup C$ is a prime diagram for the marked tangle $\mathcal{P}_{0}$, which also has the diagram $D_{0}$. If $E$ and $C$ have the same sign (defined in Section 2) as alternating tangles, then $E \cup C$ is the desired alternating representative of $\mathcal{P}_{0}$, exhibiting the appropriate crossing change. If $C$ and $D_{0}$ have opposite sign, then adjoining to $D_{0}$ the diagram $\bar{C}$ of the reverse braiding of $\mathcal{C}$ gives an alternating diagram $D_{0} \cup \bar{C}$ for the marked tangle $\mathcal{A}_{\varepsilon}(\ell, m)$, which also has the diagram $E$. Since $E$ is reduced, so is $D_{0} \cup \bar{C}$. (For, we may assume $D_{0}$ has no nugatory crossings, hence neither does $D_{0} \cup \bar{C}$. Then $E$ and $D_{0} \cup \bar{C}$ have the same crossing number. But then a reducing arc for $D_{0} \cup \bar{C}$ suggests a capping of $D_{0} \cup \bar{C}$ and of $E$ giving two alternating diagrams of 
the same link with the same number of crossings - one of which contains a nugatory crossing, the other does not). That is, $C$ is empty and $E, D_{0}$ represent the same marked tangle. Thus $E$ is the sought after diagram for $\mathcal{P}_{0}$.

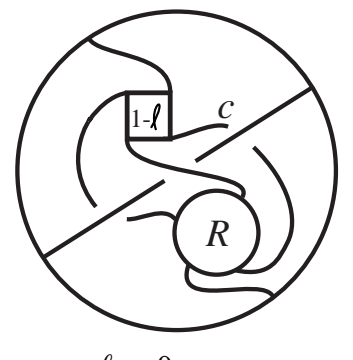

$$
\ell>0
$$

$$
R=\mathcal{R}\left(\frac{(1-\ell) m+1}{m}\right)
$$

crossing change at $c \rightarrow \mathcal{R}\left(\frac{1-2 m}{1+2 m}\right)$

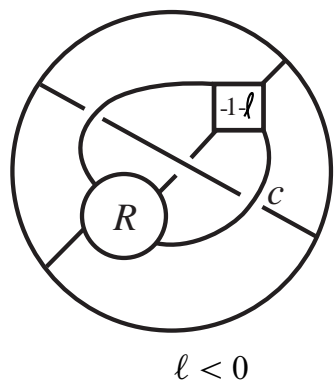

$$
R=\mathcal{R}\left(\frac{(-1-\ell) m+1}{m}\right)
$$

crossing change at $c \rightarrow \mathcal{R}\left(\frac{1-2 m}{1+2 m}\right)$

$$
\mathcal{A}_{1}(\ell, m)
$$

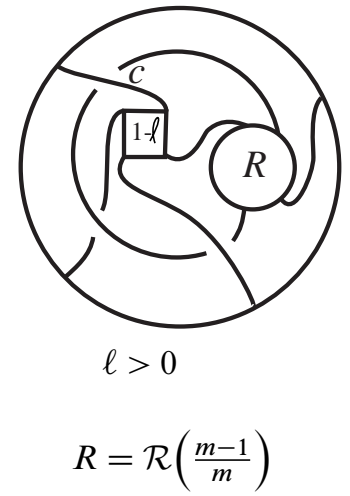

crossing change at

$$
c \rightarrow \mathcal{R}\left(\frac{(\ell-1)(1-2 m)+2}{1-2 m}\right)
$$

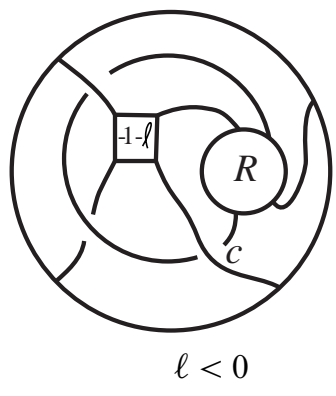

$$
R=\mathcal{R}\left(\frac{m-1}{m}\right)
$$

crossing change at

$c \rightarrow \mathcal{R}\left(\frac{2 m-1}{(-1-\ell)(2 m-1)+2}\right)$

\section{$\mathcal{A}_{2}(\ell, m)$}

Figure 12.12: Reduced, alternating diagrams of $\mathcal{A}_{\varepsilon}(\ell, m)$

Thus we assume $E$ and $C$ have opposite sign, and $C$ and $D_{0}$ have the same sign. Let $F$ be the diagram outside of $D_{0}$ in the alternating diagram of $K$. Figure 12.12 shows that after the crossing change in $E$ the sign of the rational number corresponding to $\mathcal{T}_{\mathcal{E}}(\ell, m)\left(\frac{1}{0}\right)$ is opposite to the sign of $E$. That is, the result of the crossing change has an alternating diagram $R$ such that $R \cup C$ is an alternating diagram with the same 
sign as $D_{0}$. Then the diagram $R \cup C \cup F$ is an alternating diagram of the unknot. Hence it must have a nugatory crossing. Since we may assume neither $F, C$ or $R$ contain nugatory crossings, this means that either $F$ or $R$ is a split diagram (ie, there is a properly embedded arc that separates the arcs of the tangle). Since $K$ cannot be a connected sum (by [28]), and since $F$ is not rational, $R$ must represent $\mathcal{R}(0 / 1)$ or $\mathcal{R}(1 / 0)$. But Figure 12.12 shows this is not true.

\section{Remarks about Figure 12.12}

(1) The markings have been changed between Figure 4.3 and Figure 12.12. In particular, to get from the markings in Figure 12.12 to those in Figure 4.3 add the following braiding outside of Figure 12.12:

$$
\begin{aligned}
\mathcal{A}_{1}(\ell, m), \ell>0: & -1 \text { vertical twist below } \\
\mathcal{A}_{1}(\ell, m), \ell<0: & -1 \text { vertical twist above } \\
\mathcal{A}_{2}(\ell, m), \ell>0: & +1 \text { vertical twist below, then } \\
& -1 \text { horizontal twist to left } \\
\mathcal{A}_{2}(\ell, m), \ell<0: & +1 \text { vertical twist below }
\end{aligned}
$$

(2) The diagrams for $\mathcal{A}_{1}(\ell, m)$ were obtained by the moves in Figures 12.10 and 12.11 as well as twistings that change the markings.

\section{References}

[1] S A Bleiler, A note on unknotting number, Math. Proc. Cambridge Philos. Soc. 96 (1984) 469-471 MR757839

[2] M Boileau, B Zimmermann, The $\pi$-orbifold group of a link, Math. Z. 200 (1989) 187-208 MR978294

[3] F Bonahon, L C Siebenmann, The characteristic toric splitting of irreducible compact 3-orbifolds, Math. Ann. 278 (1987) 441-479 MR909236

[4] J H Conway, An enumeration of knots and links, and some of their algebraic properties, from: "Computational Problems in Abstract Algebra (Proc. Conf., Oxford, 1967)", Pergamon, Oxford (1970) 329-358 MR0258014

[5] M Culler, C M Gordon, J Luecke, P B Shalen, Dehn surgery on knots, Ann. of Math. (2) 125 (1987) 237-300 MR881270

[6] M Eudave-Muñoz, Non-hyperbolic manifolds obtained by Dehn surgery on hyperbolic knots, from: "Geometric topology (Athens, GA, 1993)", AMS/IP Stud. Adv. Math. 2, Amer. Math. Soc. (1997) 35-61 MR1470720 
[7] M Eudave-Muñoz, On hyperbolic knots with Seifert fibered Dehn surgeries, from: "Proceedings of the First Joint Japan-Mexico Meeting in Topology (Morelia, 1999)", 121 (2002) 119-141 MR1903687

[8] C M Gordon, Dehn surgery and satellite knots, Trans. Amer. Math. Soc. 275 (1983) 687-708 MR682725

[9] C M Gordon, J Luecke, Only integral Dehn surgeries can yield reducible manifolds, Math. Proc. Cambridge Philos. Soc. 102 (1987) 97-101 MR886439

[10] C M Gordon, J Luecke, Reducible manifolds and Dehn surgery, Topology 35 (1996) 385-409 MR1380506

[11] C M Gordon, J Luecke, Non-integral toroidal Dehn surgeries, Comm. Anal. Geom. 12 (2004) 417-485 MR2074884

[12] W Haken, Theorie der Normalfächen, Acta Math. 105 (1961) 245-375 MR0141106

[13] R Hartley, Knots and involutions, Math. Z. 171 (1980) 175-185 MR570907

[14] W H Jaco, P B Shalen, Seifert fibered spaces in 3-manifolds, Mem. Amer. Math. Soc. 21 (1979) viii+192 MR539411

[15] T Kanenobu, H Murakami, Two-bridge knots with unknotting number one, Proc. Amer. Math. Soc. 98 (1986) 499-502 MR857949

[16] R Kirby, Problems in low-dimensional topology, from: "Geometric topology (Athens, GA, 1993)”, (W H Kazez, editor), AMS/IP Stud. Adv. Math. 2, Amer. Math. Soc. (1997) 35-473 MR1470751

[17] T Kobayashi, Minimal genus Seifert surfaces for unknotting number 1 knots, Kobe J. Math. 6 (1989) 53-62 MR1023526

[18] P Kohn, Two-bridge links with unlinking number one, Proc. Amer. Math. Soc. 113 (1991) 1135-1147 MR1079893

[19] W B R Lickorish, The unknotting number of a classical knot, from: "Combinatorial methods in topology and algebraic geometry (Rochester, N.Y., 1982)", Contemp. Math. 44, Amer. Math. Soc. (1985) 117-121 MR813107

[20] W H Meeks, III, P Scott, Finite group actions on 3-manifolds, Invent. Math. 86 (1986) 287-346 MR856847

[21] W Menasco, Closed incompressible surfaces in alternating knot and link complements, Topology 23 (1984) 37-44 MR721450

[22] W Menasco, M Thistlethwaite, The classification of alternating links, Ann. of Math. (2) 138 (1993) 113-171 MR1230928

[23] W Menasco, X Zhang, Notes on tangles, 2-handle additions and exceptional Dehn fillings, Pacific J. Math. 198 (2001) 149-174 MR1831976 
[24] J M Montesinos, Surgery on links and double branched covers of $S^{3}$, from: "Knots, groups, and 3-manifolds (Papers dedicated to the memory of R H Fox)", (L Neuwirth, editor), Ann. of Math. Studies 84, Princeton Univ. Press (1975) 227-259 MR0380802

[25] K Motegi, A note on unlinking numbers of Montesinos links, Rev. Mat. Univ. Complut. Madrid 9 (1996) 151-164 MR1413272

[26] Y Nakanishi, Unknotting numbers and knot diagrams with the minimum crossings, Math. Sem. Notes Kobe Univ. 11 (1983) 257-258 MR749196

[27] P Ozsváth, Z Szabó, Knots with unknotting number one and Heegaard Floer homology, Topology 44 (2005) 705-745 MR2136532

[28] M G Scharlemann, Unknotting number one knots are prime, Invent. Math. 82 (1985) 37-55 MR808108

[29] M Scharlemann, Producing reducible 3-manifolds by surgery on a knot, Topology 29 (1990) 481-500 MR1071370

[30] M Scharlemann, A Thompson, Unknotting number, genus, and companion tori, Math. Ann. 280 (1988) 191-205 MR929535

[31] M Scharlemann, A Thompson, Link genus and the Conway moves, Comment. Math. Helv. 64 (1989) 527-535 MR1022995

[32] C Sundberg, M Thistlethwaite, The rate of growth of the number of prime alternating links and tangles, Pacific J. Math. 182 (1998) 329-358 MR1609591

[33] M B Thistlethwaite, On the algebraic part of an alternating link, Pacific J. Math. 151 (1991) 317-333 MR1132393

[34] M Thistlethwaite, On the structure and scarcity of alternating links and tangles, J. Knot Theory Ramifications 7 (1998) 981-1004 MR1654669

[35] J L Tollefson, Involutions of Seifert fiber spaces, Pacific J. Math. 74 (1978) 519-529 MR0645400

[36] F Waldhausen, Über Involutionen der 3-Sphäre, Topology 8 (1969) 81-91 MR0236916

[37] Y-Q Wu, Sutured manifold hierarchies, essential laminations, and Dehn surgery, J. Differential Geom. 48 (1998) 407-437 MR1638025

[38] X Zhang, Unknotting number one knots are prime: a new proof, Proc. Amer. Math. Soc. 113 (1991) 611-612 MR1076582

Department of Mathematics, The University of Texas at Austin

1 University Station C1200, Austin, TX 78712-0257, USA

gordon@math.utexas.edu, luecke@math.utexas.edu

Received: 9 January 2006

Algebraic $6 \mathcal{G}$ Geometric $\mathcal{T}$ opology, Volume 6 (2006) 